\title{
MiCHEL GROS
}

\section{Classes de Chern et classes de cycles en cohomologie de Hodge-Witt logarithmique}

Mémoires de la S. M. F. $2^{e}$ série, tome 21 (1985)

<http://www.numdam.org/item?id=MSMF_1985_2_21_1_0>

(C) Mémoires de la S. M. F., 1985, tous droits réservés.

L'accès aux archives de la revue « Mémoires de la S. M. F. » (http://smf. emath.fr/Publications/Memoires/Presentation.html) implique l'accord avec les conditions générales d'utilisation (http://www.numdam.org/conditions). Toute utilisation commerciale ou impression systématique est constitutive d'une infraction pénale. Toute copie ou impression de ce fichier doit contenir la présente mention de copyright.

\section{Numdam}


Mémoire de la Societé Mathématique de France, $\mathrm{n}^{0} \cdot 21$

Supplément au Bulletin de la S.M.F., Tome 113, 1985, tascicule 4

CLASSES DE CHERN ET CLASSES DE CYCLES

EN COHOMOLOGIE DE HODGE-WITT LOGARITHMIQUE

MICHEL GROS (*)

Résumé

Si $k$ est un corps parfait de caractéristique $p>0$, on définit, pour tout $k$-schéma lisse $\mathrm{X}$, une thérie de classes de Chern et de classes de cycles à valeurs dans les groupes de cohomologie étale $H^{i}\left(x_{e t}, w_{n} \Omega_{l o g}^{i}\right)$, ou $W_{n} \Omega_{x}^{i}$ est le complexe de de Rham-Witt de cran $n$ de $x$ et $w_{n} \Omega^{i}$ log le sousfaisceau abélien de $w_{n} \Omega_{x}^{i}$ engendré localement, pour la topologie étale, par les différentielles logarithmiques. Via l'application naturelle de $\mathrm{H}^{i}\left(\mathrm{x}_{e t}, \mathrm{w}_{n} \Omega^{i} \log \right)$ dans le groupe de cohomologie cristalline $\mathrm{H}_{\mathrm{Cris}}^{2 i}\left(\mathrm{X} / \mathrm{W}_{n}\right)$, on retrouve les classes de Chern de Berthelot-Illusie, et la classe de cohamologie d'un sous-schéna fermé lisse construite par Berthelot. Notre construction de la classe de cohomologie d'un cycle repose sur un thérème de pureté pour les faisceaux $W_{n} \Omega^{i} \log$. On montre que cette classe transforme intersections propres en cup-produits, et l'on prouve la compatibilité usuelle entre classe de Chern et classe de cycle pour un sous-schéma fermé (non nécessairement lisse).

\section{Abstract}

Let $k$ be a perfect field of characteristic $p>0$. For every smooth $k$-scheme $x$, we define a theory of chern classes and cycle classes with value in the étale cohomology groups $H^{i}\left(x_{e t}, w_{n} \Omega^{i} l o g\right)$, where $w_{n} \Omega_{\dot{X}}^{i}$ is the Rham-witt complex of $x$ of step $n$ and $w_{n} \Omega^{i}$ log the abelian subsheaf of $w_{n} \Omega_{x}^{i}$ generated locally for the étale topology by the logarithmic differentials. Via the natural map from $H^{i}\left(x_{e t}, w_{n} \Omega^{i} l_{0 g}\right)$ to the crystalline cohomology group $H_{c r i s}^{2 i}\left(x / w_{n}\right)$, we recover Berthelot-Illusie's Chem classes and the cohomology class of a smooth closed subscheme constructed by Berthelot. Our construction of the cycle class relies on a purity theorem for the sheaves $w_{n} \Omega^{i} \log$. Wh show that proper intersections correspond to cup-products and we prove the usual compatibility between the Chern class and the cycle class of a (non necessarily smooth) closed subscheme.

Texte reçu le 15 avril 1985

(*) Thèse de doctorat de $3 e$ cycle de Mathématiques, Université de Paris-sud, Centre d'Orsay, juin 83. 
TABLE DES MATIÉRES

INTRODUCTION ........................................4

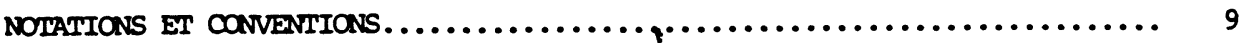

I. COHOMOLOGIE DES FIBRÉS PROJECTIFS.

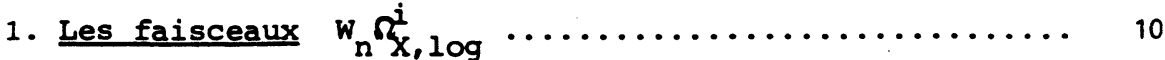

2. Cohomologie logarithmique des fibrés projectifs....... 12

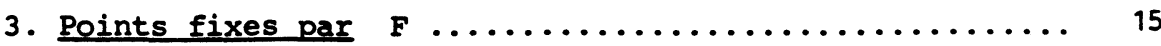

4. Cohomoloqie de Hodqe-Witt des fibrés proiectifs....... 19

5. Comparaison avec la cohomologie cristalline......... 23

II. MORPHISME DE GYSIN.

1. Définition des différents morphismes de Gysin....... 27

1.1. Rappels sur l'autodualité du complexe de De Rham-Witt.

1.2. Définitions.

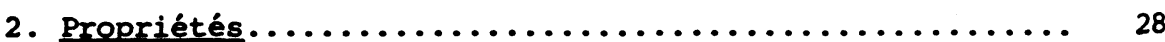

2.1. Transitivité.

2.2. Formule de projection.

2.3. Transversalité.

2.4. Formule d'intersection.

2.5. Classe de cycle et classe de Chern.

2.6. Cas d'un morphisme $P(E) \longrightarrow X$.

2.7. Cas d'un morphisme birationnel.

3. Cas d'une imersion fermée.................... 38

3.1. Préliminaires d'algèbre homologique.

3.2. Construction locale du morphisme de Gysin.

3.3. Description du morphisme de Gysin.

3.4. Comparaison avec la définition 1.2 .6 .

3.5. Cas de la cohomologie logarithmique.

4. Classe de cohomologie d'un cycle................. 50

4.1. Définition.

4.2. Propriétés.

5. Morphisme de Gysin et complexe de Cousin............

5.1. Cas de la cohomologie de Hodge-Witt.

5.2. Cas de la cohomologie logarithmique. 
III. CLASSES DE CHERN LOGARITHMIQUES.

1. Classes de Chern logarithmiques...$\ldots \ldots \ldots \ldots \ldots .60$ 1.1. Enoncé du théorème principal.

1.2. Démonstration du théorème principal.

2. Variantes et compatibilités..................... 65

2.1. Comparaison avec les classes de Chern cristallines.

2.2. Cohomologie d'un schéma de drapeaux.

Appendice : classes de Chern à supports............ 68

IV. APPLICATIONS.

1. Cohomologie d'un éclaté $. . . \ldots \ldots \ldots \ldots \ldots \ldots \ldots \ldots \ldots \ldots \ldots \ldots, 71$

1.1. Cohomologie de Hodge-Witt d'un éclaté.

1.2. Cohomologie de Hodge d'un éclaté.

1.3. Cohomologie logarithmique d'un éclaté.

2. Formule de self-intersection.................... 78

3. Classe de Chern et classe de cycle................ 84

BIBLIOGRAPHIE. 


\section{INTRODUCTION}

Soient $k$ un corps parfait de caractéristique $p>0$ et $x$ un k-schéma lisse. Si $\mathrm{Y}$ est un sous-schéma fermé lisse de $\mathrm{X}$ de codimension $r$, on sait (cf. [2]) lui associer une classe fondamentale de cohomologie $\mathrm{cl}(\mathrm{Y} / \mathrm{X})$ dans la cohomologie cristalline $\mathrm{H}_{\mathrm{cris}}^{2 \mathrm{r}}(\mathrm{X} / \mathrm{W}(\mathrm{k}))$ de $\mathrm{X}$ sur les vecteurs de Witt $W(k)$ de $k$. Un des objets du présent travail est de s'affranchir de l'hypothèse de lissité faite sur $Y$ en employant des techniques afférentes au complexe de De Rham-Witt ([21]).

Des exemples tels ceux de la cohomologie étale à valeurs dans $\mathbf{z}_{\chi}(\chi \neq p)$ ou de la cohomologie de De Rham en caractéristique 0 montrent que la définition de la classe de cohomologie d'un cycle éventuellement singulier est possible dès que $l^{\prime}$ 'on dispose $d$ un théorème de pureté permettant $d$ 'exprimer de manière plus simple certains groupes de cohomologie à support. Il est bien connu (cf. [2]) que la cohomologie cristalline ne vérifie pas de tels théorèmes de pureté. On peut toutefois travailler avec des groupes de cohomologie "plus fins", tels que les groupes de cohomologie logarithmiques $H^{*}\left(X_{e t}, w_{n} \Omega^{*}, \log \right)$, où les faisceaux étales $w_{n} \Omega^{*}, \log$ sont les faisceaux définis dans [21] (ils diffèrent. très légèrement des faisceaux $\nu_{n}(i)$ introduits par Milne dans [28]).

En effet, on verra que ces faisceaux vérifient des propriétés de pureté suffisantes pour permettre une définition satisfaisante de la classe de cohomologie d'un cycle éventuellement singulier. Comme ils semblent d'autre part jouer un rôle important dans diverses questions de géométrie arithmétique (cf. [5], [7], [25]), il nous a paru utile de donner certaines constructions et théorèmes concernant ces faisceaux : classes de Chern, classes de cycles, cohomologie d'un schéma éclaté, formule de self-intersection, théorème de Riemann-Roch pour une immersion fermée. 
Le chapitre I est essentiellement consacré au calcul de $R f_{*} W_{n} \Omega_{p}^{*}$ et $R f_{*} W_{n} \Omega_{p, l o g}^{*}$ où $P$ est le fibré projectif associé à un $\theta_{X}$-Module localement libre de rang $r+1$ et $f$ la projection canonique $P \longrightarrow X$. Par analogie avec le calcul de $R_{*} \Omega_{p}$ (cf. [4] ou [14]), on construit des morphismes canoniques dans des catégories dérivées convenables

$$
\begin{aligned}
& \underset{i=0}{\mp} w_{n} \Omega_{x}(-i)[-i] \longrightarrow R_{*} w_{n} \Omega_{p} \\
& \bigoplus_{i=0} w_{n} \Omega_{x, \log }^{*}(-i)[-i] \longrightarrow R f_{*} w_{n} \Omega_{p, \log }^{*} \cdot
\end{aligned}
$$

dont on montre que ce sont des isomorphismes. Grosso modo, (2) se déduit de (1) en prenant "les points fixes sous $F$ ". La vérification que (1) est un isomorphisme utilise le "lemme de Nakayama d'Ekedahl" (cf. [9], [22]) pour se ramener au cas $n=1$.

Au chapitre II, nous développons une théorie du morphisme de Gysin pour des morphismes propres entre $\mathrm{k}$-schémas lisses. Après un rappel des résultats de dualité du complexe de De Rham-Witt dus à T. Ekedahl, nous construisons pour tout morphisme propre $\mathrm{f}: \mathrm{Y} \longrightarrow \mathrm{X}$ entre k-schémas lisses, un morphisme de Gysin

$$
f_{*}: R f_{*} W \Omega_{Y} \longrightarrow w \Omega_{X}(r)[r] \quad(r=\operatorname{dim}(X)-\operatorname{dim}(Y))
$$

dans la catégorie dérivée des $R_{X}$-Modules où $R_{X}$ est un certain faisceau d'anneaux sur $X$ défini par $T$. Ekedahl et généralisant l'anneau $R$ de [23]. Par application d'un foncteur "points fixes sous $F^{\prime \prime}$, on en déduit un morphisme

$$
f_{*}: R f_{*} W \cdot \Omega_{Y, \log }^{*} \rightarrow W \cdot \Omega_{X, \log }^{*}(r)[r]
$$

dans la catégorie dérivée des W. $\Omega_{x, l o g}^{*}$-pro-Modules.

Au numéro 2, on énonce diverses propriétés de (4) : transitivité, formule de projection, etc.

Le numéro 3 est indépendant des résultats de $T$. Ekedahl. On propose dans le cas où $f$ est une immersion fermée, une construction d'un morphisme

$$
w_{n} \Omega \dot{Y} \rightarrow \mathbb{H}_{Y}^{-\Gamma}\left(w_{n} \Omega_{X}^{*}(r)\right)
$$


M. GROS

à l'aide de relèvements locaux $Y^{\prime}$ et $X^{\prime}$ de $Y$ et $X$ sur $W_{n}$ et du morphisme de Gysin en cohomologie de De Rham (cf. [2])

$$
\Omega_{Y}^{\prime} / w_{n} \longrightarrow \underline{H}_{Y}^{\cdot I}\left(\Omega_{X}^{*} / w_{n}(r)\right) \text {. }
$$

Ceci est rendu possible par l'utilisation de l'isomorphisme (cf. [23])

$$
w_{n} \Omega_{X}^{i} \stackrel{\sim}{\longrightarrow}\left(\Omega_{X}^{*} / w_{n}\right)
$$

On vérifie que le morphisme ainsi construit est bien identique à celui qu'on déduit de ( 3 ).

Par application du foncteur "points fixes sous F", (6) fournit un morphisme

$$
\left.w_{n} \Omega_{Y, \log }^{*} \rightarrow \mathrm{H}_{Y}^{*}{ }^{*} w_{n} \Omega_{X, \log }^{*}(r)\right)
$$

qui est en fait un isomorphisme.

Ce morphisme diffère des morphismes que $l^{\prime}$ on peut déduire de (4), mais l'on peut récupérer (4) à l'aide de (8) et démontrer ainsi certaines des propriétés énoncées au numéro 2.

Le numéro 4 donne la définition de la classe d'un cycle éventuellement singulier de $x$ (construction faite indépendamment par Milne dans [30]). La méthode est assez proche du cas de la cohomologie de De Rham en caractéristique 0 , à ceci près qu'ici, on n'a pas $H_{Y}^{r+1}\left(w_{n} \Omega_{X, l o g}^{*}\right)=0$. On termine ce numéro en prouvant la compatibilité de la classe ainsi construite aux intersections, puis en prouvant que deux cycles algébriquement équivalents ont même classe de cohomologie.

Au numéro 5, nous revenons au cas d'un morphisme propre quelconque et montrons comment réaliser (3) à l'aide de complexes de Cousin.

Le chapitre III est consacré à la définition des classes de Chern $c_{i}(E) \in H^{i}\left(x, w_{n} R_{x}^{i}, \log ^{\prime}\right)$ attachées à tout $\theta_{x}$-Module localement libre de rang fini. Compte tenu des résultats du premier chapitre et de la théorie du morphisme de Gysin pour une immersion fermée (y compris la compatibilité à la première classe de Chern dans le le cas de la codimension 1), les méthodes sont essentiellement standard (cf. [13] et [SGA 5, VII]). 
Le numéro 2 est consacré à la comparaison des classes ainsi définies avec les classes de Chern cristallines de [4]. On termine le chapitre par le calcul de la cohomologie d'un schéma de drapeaux.

Au chapitre IV, nous calculons la cohomologie d'un schéma éclaté par des techniques analogues à celles utilisées pour prouver que (1) et (2) sont des isomorphismes. On en déduit la "formule de selfintersection"

$$
i^{*} i_{*}(y)=y c_{r+1}(\check{N}) \in H^{r+1}\left(x, w_{n} \sigma_{x, l o g}^{r+1}\right)
$$

pour $i$ une immersion fermée lisse de codimension $r+1$ définie par un idéal $\mathrm{J}$ de $\theta_{X}$ et $\mathrm{N}=\mathrm{J} / \mathrm{J}^{2}$.

Nous prouvons également une variante de la "formule-clef" de ([SGA 5, VII]) et de la formule "habituelle" comparant classe de Chern et classe de cycle

$$
c_{r+1}\left(i_{*} \theta_{Y}\right)=(-1)^{r} r ! c l(Y / X) \in H^{r+1}\left(X, w_{n} \Omega_{X, l o g}^{r+1}\right) .
$$

C'est une conséquence facile du théorème de Riemann-Roch pour une immersion fermée dont la démonstration est essentiellement la même qu'en $[24]$.

Ce travail où intervient de manière essentielle la théorie du complexe de De Rham-Witt et des $R_{X}$-Modules $m$ 'a été suggéré par L. Illusie. Je tiens à lui exprimer ici toute ma gratitude pour la patience avec laquelle il $\mathrm{m}$ 'a prodigué conseils et encouragements tout au long du travail.

P. Berthelot et $T$. Ekedahl ont bien voulu lire une première version de la thèse et corriger certaines erreurs. Qu'ils en soient ici chaleureusement remerciés. Je remercie également 0 . Gabber qui m'a communiqué certains résultats qui ont servi de catalyseur à ceux présentés ici. 
M. GROS

(Ajouté en octobre 1985) Nous venons de prendre connaissance d'un preprint de H. Gillet et W. Messing intitulé "Cycle Classes and Riemann-Roch for Crystalline cohomology", donnant, pour $X$ lisse sur un corps $k$ de caractéristique $\mathrm{p}>0$, une thérie de classes de cycles à valeurs dans les groupes de cohomologie cristalline $\mathrm{H}_{\text {cris }}^{2 i}(\mathrm{X} / \mathrm{C}) \bullet \mathbb{Q}(\mathrm{C}=$ anneau de Cohen pour $k)$. Pour $k$ parfait (et $C=W$ ), cette theorie se déduit de celle construite ici via l'appli-

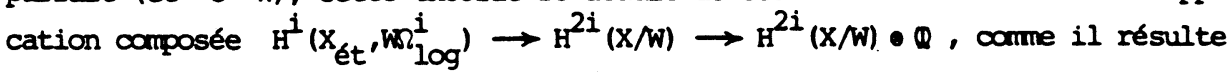
de III 2.1 et IV 3. 
Dans tout ce travail, on fixe un nombre premier $p$ et un corps parfait $k$ de caractéristique $p$. On note

$$
\begin{aligned}
& W(k) \text { (ou } W \text { ) : I'anneau des vecteurs de Witt sur } k \text {, } \\
& w_{n}(k) \text { (ou } w_{n} \text { ): } w(k) / p^{n} \text {, l'anneau des vecteurs de witt de } \\
& \text { longueur } \mathrm{n} \text {. }
\end{aligned}
$$

Si $\mathrm{X}$ est un $\mathrm{k}$-schéma, on note :

$$
\begin{array}{ll}
G_{\mathrm{m}}=\theta_{\mathrm{X}}^{*} & \text { le groupe multiplicatif sur } \mathrm{X} \\
\Omega_{\mathrm{X}} & \text { le complexe de De Rham de } \mathrm{X} \text { sur } k
\end{array}
$$$$
W_{n} \Omega_{i} \text { le complexe de De Rham-Witt de } x \text { de cran } n
$$$$
\text { ([21], I 1.12) }
$$

W. $\Omega_{\mathrm{X}}$ le système projectif (ou parfois le pro-objet) formé par les $w_{n} \Omega_{\dot{x}}$ et les flèches de restriction. $w_{n+1} \Omega_{x} \rightarrow w_{n} \Omega_{x}$. notées $\pi$.

$w \Omega_{x}:$ lim $w_{n} \Omega_{x}$ le complexe de De Rham-Witt de $x$ $\underline{x}=(x, 0,0, \ldots) \in w \theta_{x} \quad\left(\right.$ ou $\left.w_{n} \theta^{\prime}\right)$ le représentant de Teichmuller de $x \in \theta_{x}$.

$S i \mathrm{M}$ est un complexe ( $d$ 'une catégorie abélienne), on note $M^{\langle i}, M^{\rangle_{i}}$ les tronqués naifs de $M$ et $\left.t_{\leqslant_{i}} M, t\right\rangle_{i} M$ les tronqués canoniques de $M$ :

$M^{S_{i}}=\left(\ldots \rightarrow M^{i-1} \longrightarrow M^{i} \longrightarrow 0\right), M^{\rangle_{i}}=\left(0 \longrightarrow M^{i} \longrightarrow M^{i+1} \longrightarrow \ldots\right)$

$t_{\leqslant_{i} M}=\left(\ldots \rightarrow M^{i-1} \longrightarrow z^{i} M \longrightarrow 0\right), t_{\geqslant_{i} M}=\left(0 \longrightarrow M^{i} / B^{i} M \longrightarrow M^{i+1} \rightarrow \ldots\right)$

où $\left.z_{M}^{i}=\operatorname{Ker} d, B^{i} M=I m d\right)$.

Si $P^{\cdot}$ est un objet gradué et $s i \quad r \in \mathbb{Z}, P^{*}(r)$ désigne l'objet gradué défini par $\left(P^{\bullet}(r)\right)^{i}=P^{r+i}$.

Si I est un faisceau, la notation $x \in L$ signifie que $x$ est une section locale de $\mathrm{L}$.

L'écriture $a:=b$ signifie que a est défini par $b$. 


\section{CHAPITRE I}

\section{COHOMOLOGIE DES FIBRÉS PROJECTIFS}

Dans tout ce chapitre, la lettre $\mathrm{x}$ désignera un $\mathrm{k}$-schéma lisse.

1. Les faisceaux $w_{n} \Omega_{x, 10 g}^{i}$.

1.1. Pour $n \geqslant 1$, nous noterons

$$
(1.1 .1) \quad \operatorname{dlog}: \theta_{x}^{*} \rightarrow w_{n} \Omega_{x}^{1}
$$

l'homomorphisme de faisceaux abéliens défini par $x \longmapsto d x / x$.

Pour $n$ variable, les applications (1.1.1) forment un homomorphisme du système projectif constant $\theta_{X}^{*}$ dans le système projectif $W . \Omega_{X}^{1}$ que nous noterons encore dlog.

1.2. Soit $i \geqslant 1$, on rappelle ([21], I.5.7) que 1 'on désigne par $w_{n} \Omega_{x}^{i} \log$ le sous-faisceau abélien de $w_{n} \Omega_{x}^{i}$ engendré localement, pour la topologie étale sur $x$, par les sections de la forme $\operatorname{d} \log \left(\underline{x}_{1}\right) \ldots \operatorname{dlog}\left(x_{i}\right)$ avec $x_{1}, \ldots, x_{i} \in \theta_{x}^{*}$ si $n \geqslant 1$ et $w_{n} \Omega_{x, l o g}^{i}=0$ si $n \leqslant 0$. Pour $i=0$, on conviendra que $w_{n} \Omega_{x, \log }^{0}=2 / p^{n}$ si $n>0$ et $w_{n} \mathcal{R}_{x, l o g}=0$ si $n \leqslant 0$.

Lorsque $n=1$, on écrira $\sum_{x, l o g}^{i}$ au lieu de $w_{1} \Omega_{x, l o g}^{i}$.

Chaque fois que $l^{\prime}$ on écrira $w_{n} \Omega_{x}^{i} \log$, il sera sous-entendu que l'on travaille sur le site étale des schémas considérés.

Si $\mathrm{f}: \mathrm{X} \longrightarrow \mathrm{X}$ ' est un morphisme de k-schémas lisses, les morphismes canoniques de fonctorialité ([21], I (1.2.12), (1.2.13)) induisent des morphismes canoniques adjoints $l$ 'un de l'autre

$$
\begin{aligned}
& w_{n} \Omega_{x}^{*}, \log \longrightarrow f_{*} w_{n} \Omega_{x, 10 g}^{*} \\
& f^{-1} w_{n} \Omega_{x^{\prime}, \log }^{*} \rightarrow w_{n} \Omega_{x, \log }^{*} \text {. }
\end{aligned}
$$


La structure de produit de $w_{n} \Omega_{x}^{*}$ induit une structure de produit associatif et anticommutatif sur $w_{n} \Omega_{x}^{*}, 10 g$

$$
w_{n} \Omega_{x, \log }^{i} \times w_{n} \Omega_{x, \log }^{i} \rightarrow w_{n} \Omega_{x, l o g}^{i+j} \text {. }
$$

1.3. Les résultats essentiels dont nous aurons besoin sont les suivants :

On a une suite exacte (pour la topologie étale sur $X$ ) (Thm. 2.4 .2 de $[21]$ )

$$
(1.3 .1) \quad 0 \longrightarrow \Omega_{x, 10 g}^{i} \longrightarrow \mathrm{z} \Omega_{x}^{i} \underset{1-C_{x}}{~} \Omega_{x}^{i} \longrightarrow 0 \text {. }
$$

Ce résultat se généralise de la manière suivante : on a une suite exacte de pro-faisceaux abéliens (pour la topologie étale sur $\mathrm{X}$ )

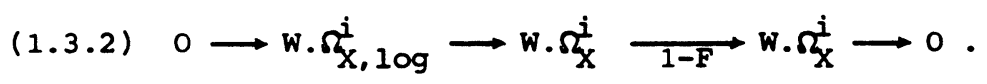

C'est le théorème I.5.7.2 de [21].

Les catégories dérivées avec lesquelles nous aurons à travailler sont les suivantes : $D\left(z / p^{n}-M o d\right), D\left(z / p^{*}\right.$-pro-Mod $), D\left(w_{n} \Omega_{X}^{*}, \log ^{-M o d}\right)$,

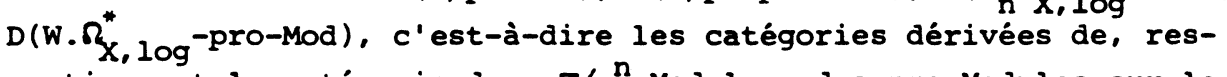
pectivement la catégorie des $\mathrm{Z} / \mathrm{p}^{\mathrm{n}}$-Modules, des pro-Modules sur le le pro-Anneau $z / p^{*}$, des $w_{n} \Omega_{x, \log }^{*}$-Modules $\left(w_{n} \Omega_{x, l o g}^{*}\right.$ étant un anneau d'après ce qui précède) gradués, des pro-Modules gradués sur le pro-anneau gradué $\mathrm{W} . \Omega_{\mathrm{X}}^{*}, \mathrm{log}$.

Théorème 1.3.3. La flèche naturelle de $D\left(w_{n} \Omega_{x, \log }^{*}\right.$-pro-Mod $)$

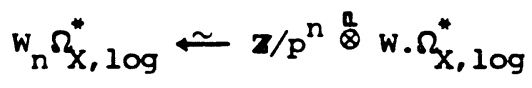

est un isomorphisme.

Démonstration : Il suffit de vérifier qu'elle induit un isomorphisme pour tout $i \geqslant 0$

$$
w_{n} \Omega_{x, l o g}^{i} \stackrel{\sim}{2} / p^{n} \stackrel{n}{\otimes} \cdot \Omega_{x, l o g}^{i}
$$

dans $D\left(z / p^{n}\right.$-pro-Mod). Or, on a un isomorphisme de $z / p^{n}$-pro-Modules

$$
\text { (1.3.6) } \quad w_{n} \Omega_{x, \log } \simeq z / p^{n} \otimes w . \Omega_{x, l o g}^{i}
$$


d'après le corollaire 5.7 .5 de [21]

D'autre part, on a

$$
\operatorname{Tor}_{j}^{z}\left(z / p^{n}, w \cdot R_{x, l o g}^{i}\right)=0
$$

pour $j>0$ et tout $i$ car la multiplication par $p$ est injective dans le pro-objet $w . r_{x}^{i}, \log$ puisque c'est déjà le cas sur le proobjet w. $\Omega_{X}^{i}$ d'après le corollaire I.3.5 de [21].

\section{Cohomoloqie logarithmique des fibrés projectifs.}

2.1. Soit $E$ un $\theta_{X}$-Module localement libre de rang $r+1$ $(r \geqslant-1)$. On notera $P=P(E)$ le fibré projectif sur $X$ associé à $E$ et $f$ la projection $P \longrightarrow X$. On veut calculer $R f_{*} W_{n} \Omega_{p, l o g}^{*}$

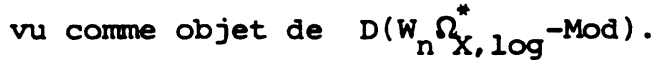

on remarque que (1.1.1) fournit un morphisme

$$
\text { dlog : } \theta_{p}^{*} \longrightarrow \lim \text { W. } \Omega_{p, l o g}^{1}
$$

qui, composé avec 1 'homomorphisme canonique $l i m \mathrm{~W} . \Omega_{\mathrm{p}}^{1}, \log$ Rlim W. $\Omega_{\mathrm{P}, \log }^{1}$ fournit un morphisme de $D(z)$

$$
\text { dlog }: \theta_{P}^{*} \longrightarrow \text { Rlim W. } \Omega_{P, l o g}^{1}
$$

lequel induit en cohomologie un morphisme

$$
\text { (2.1.3) } c_{1}: \operatorname{Pic}(\mathrm{P}) \stackrel{\sim}{\longrightarrow} \mathrm{H}^{1}\left(\mathrm{P}, \theta_{\mathrm{P}}^{*}\right) \longrightarrow \mathrm{H}^{1}\left(\mathrm{P}, \mathrm{RLim} \mathrm{W} . \Omega_{\mathrm{P}, \mathrm{log}}^{1}\right) .
$$

Pour tout faisceau inversible $L$ sur $P, C_{1}(L)$ désignera l'image par (2.1.3) de la classe de $L$ dans Pic(P) et sera appelée première classe de Chern (logarithmique) de $L$.

Pour tout objet $M$. de $D\left(\mathbf{z} / \mathrm{p}^{\circ}\right.$-pro-Mod), on a un isomorphisme canonique évident

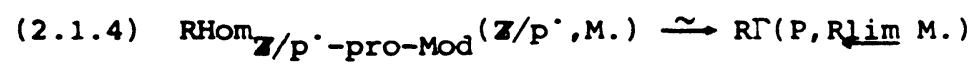

8 i bien que

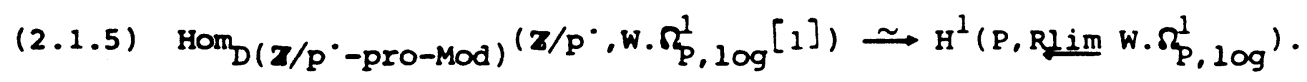




\section{COHOMOLOGIE DES FIBRÉS PROJECTIFS}

L'élément $c_{1}\left(\theta_{P}(1)\right)$ de $\mathrm{H}^{1}\left(P, R_{1}\right.$ im $\left.W . \Omega_{P}^{1}, \log ^{\prime}\right)$ définit donc un morphisme dans $D\left(x / p^{\circ}\right.$-pro-Mod) noté encore $c_{1}\left(\theta_{P}(1)\right)$

$$
\text { (2.1.6) } \quad c_{1}\left(\theta_{\mathrm{P}}(1)\right): \mathrm{z} / \mathrm{p}^{\cdot} \longrightarrow \mathrm{w} \cdot \Omega_{\mathrm{p}, \log }^{1}[1] .
$$

La structure de produit (1.2.3) permet, par produit tensoriel, de déduire de $(2.1 .6)$ des morphismes dans $D\left(z / p^{\circ}\right.$-pro-Mod $)$

$$
c_{1}\left(\theta_{p}(1)\right)^{i}: z / p \rightarrow w . \delta_{p}^{i}, \log ^{[i]}
$$

et ceci pour tout $i \geqslant 0 \quad\left(c_{1}\left(\theta_{P}(1)\right)^{0}=I d\right)$.

On en déduit par adjonction un morphisme dans $D\left(W . \Omega_{P}^{*}, \log { }^{- \text {pro-Mod })}\right.$

$$
\text { (2.1.8) } \underset{i=0}{\stackrel{r}{\oplus}} c_{1}\left(\theta_{P}(1)\right)^{i}: \underset{i=0}{\oplus} w \cdot \Omega_{P, \log }^{*}(-i)[-i] \longrightarrow w \cdot \Omega_{p, \log }^{*}
$$

Pour tout module différentiel gradué $M^{*}$ (ou tout complexe $M^{*}$ ), on note $M^{*}(m)[n]$ (ou $M^{*}(m)[n]$ ) le module différentiel gradué (ou le complexe) obtenu en appliquant $m$ fois le foncteur translation pour les modules différentiels gradués (ou pour les complexes) et $\mathrm{n}$ fois le foncteur translation dans la catégorie dérivée que l'on considère.

Enfin, utilisant le morphisme d'adjonction

$$
\operatorname{adj}: \text { W. } \Omega_{X, \log }^{*} \longrightarrow R f_{*} W \cdot \Omega_{P, l}^{*} \log .
$$

on obtient un morphisme dans $D\left(W . \Omega_{X}^{*}, \log\right.$-pro-Mod)

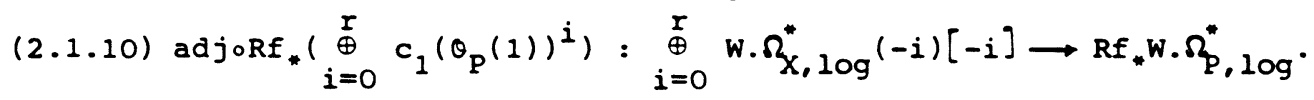

Théorème 2.1.11. Le morphisme $(2.1 .10)$ est un isomorphisme.

$\mathrm{Au} \mathrm{n}^{\circ} 4$, nous déduirons ce théorème d'un théorème analogue concernant la cohomologie de Hodge-Witt d'un fibré projectif. Nous donnerons cependant au numéro 2.2 une démonstration directe.

Corollaire 2.1.12. On a pour tout $n \geqslant 0$ un isomorphisme canonique dans $D\left(W_{n} \Omega_{x, l o g}^{*}{ }^{-M o d}\right)$

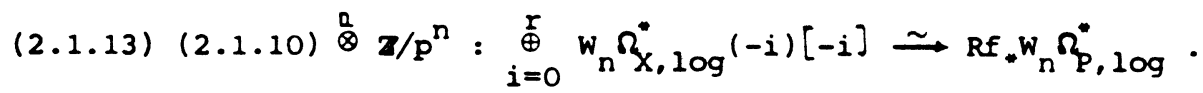


M. GROS

Démonstration : On applique la formule de projection

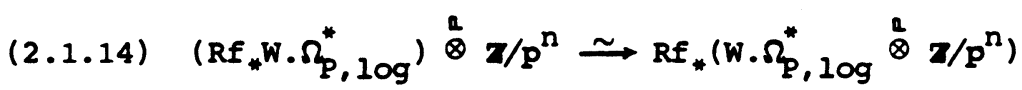

et 1 'isomorphisme $(1.3 .4)$.

corollaire $2 \cdot 1.15$. On a un isomorphisme de $\mathrm{z} / \mathrm{p}^{\mathrm{n}}$-Modules

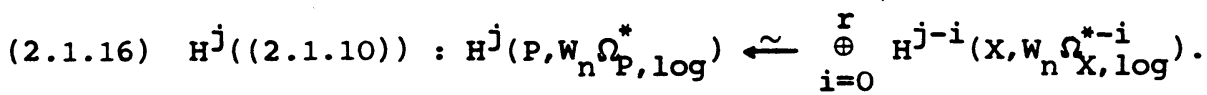

Démonstration : Il suffit d'appliquer le foncteur $H^{j}\left(x_{,}-\right)$à

I'isomorphisme (2.1.10).

Corollaire 2.1.17. On a

(2.1.18) $H^{j}\left(P_{k}^{r}, w_{n} \Omega_{P_{k}^{r}, l o g}^{i}\right)=\left\{\begin{array}{l}0 \text { pour } i \neq j \text { ou } i\rangle_{r} \\ z / p^{n} \cdot c_{1}\left(\theta_{P_{k}^{r}}(1)\right)^{i} \text { pour } i \in[0, r]\end{array}\right.$

Démonstration : Il suffit d'appliquer le corollaire précédent au cas $\mathrm{X}=\operatorname{Spec}(\mathrm{k})$ et $\mathrm{E}=\mathrm{k}^{\mathrm{r+1}}$.

2.2. Démonstration de 2.1.11. On commence par remarquer que l'isomorphisme (1.3.4) appliqué à chacun des deux membres montre que Cône((2.1.10)) ${ }^{\mathrm{Q}} \mathrm{Z} / \mathrm{p}^{\mathrm{n}} \stackrel{\mathrm{C}}{\longrightarrow} \mathrm{Cône}(2.1 .13)$. Soit $c^{*}$. le cône de (2.1.10), il suffit donc de démontrer que $c . \stackrel{\otimes}{\otimes} / \mathrm{p}=0$, car alors

$c^{*} \mathrm{z}^{2} \mathrm{z} \mathrm{p}^{\mathrm{n}}=0$ et donc $c_{.}^{*}=0$. On remarque alors que $c^{*} \stackrel{2}{\otimes} \mathrm{z} / \mathrm{p}$ est le cône du morphisme (2.1.13) pour $n=1$. On vérifie alors que la suite exacte $(1.3 .1)$ permet de définir un morphisme de triangles distingués

(2.2.1)

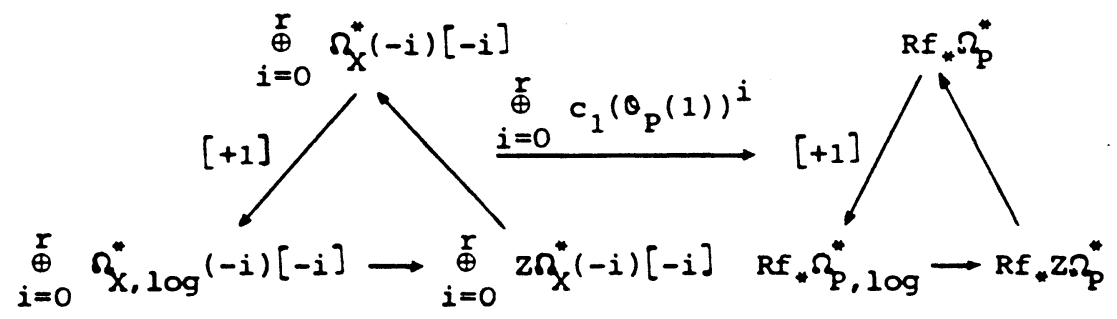

(1) Pour $n=1$, ce résultat a été prouvé indépendamment par 0 . Gabber (non publié, cf. [15]). 
(la définition des morphismes horizontaux étant analogue à la définition du morphisme (2.1.10)).

Comme le morphisme

$$
\text { (2.2.2) } \underset{i=0}{\stackrel{\Gamma}{\oplus}} c_{1}\left(\theta_{P}(1)\right)^{i}: \underset{i=0}{\mathbb{\oplus}} \Omega_{X}^{*}(-i)[-i] \longrightarrow \operatorname{Rf}_{*} \Omega_{P}^{*}
$$

est un isomorphisme $([4]$ et $[14])$, il suffit de démontrer que

$(2.2 .3) \underset{i=0}{\oplus} c_{1}\left(\theta_{P}(1)\right)^{i}: \underset{i=0}{\oplus} z \Omega_{X}^{*}(-i)[-i] \longrightarrow \operatorname{Rf}_{*} z \Omega_{P}^{*}$

est un isomorphisme.

On utilise pour cela un argument qui m'a été communiqué par L. Illusie (cf. aussi $[31,1.5]$ ). On commence par prouver que 1 'on a un isomorphisme

$$
(2.2 .4) \underset{i=0}{\oplus} c_{1}\left(\theta_{P}(1)\right)^{i}: \underset{i=0}{T} t_{\leqslant j-i} \Omega_{X} \rightarrow R_{*} t_{S j} \Omega_{P}
$$

en remarquant pour ce faire que le conoyau de l'application injective $t_{\leqslant j} \delta_{X}^{\circ} \longrightarrow t_{\leqslant j+1} \Omega_{X}^{j}$ est quasi-isomorphe à $\Omega_{X}^{j+1}[-(j+1)]$ grâce à 1 'opération de Cartier. Par récurrence ascendante sur $j$, on déduit alors de (2.2.2) que $(2.2 .4)$ est un isomorphisme.

Pour terminer, on remarque que $z \Omega_{\mathrm{X}}^{j}[-j]$ est le noyau du morphisme surjectif $t_{\langle j} \Omega_{X} \rightarrow \Omega_{X}^{k j}$ et donc qu'il suffit de prouver que $(2.2 .5) \underset{i=0}{\oplus} c_{1}\left(\theta_{p}(1)\right)^{i}: \underset{i=0}{T} Q^{k j-i}[-i] \longrightarrow R f_{*} \Omega_{p}^{\leqslant j}$ est un isomorphisme. Ce qui résulte facilement de l'isomorphisme $(2.2 .2)$.

\section{Points fixes par $F$.}

3.1. Commençons par rappeler la définition de l'anneau $R_{X}$. généralisation due à $T$. Ekedahl de l'anneau de Cartier-DieudonnéRaynaud. L'anneau $R_{X}$ est par définition la $w \Omega_{X}^{*}$-algèbre différentielle graduée engendrée par deux générateurs $\underline{F}$ et $\underline{v}$ de degré 0

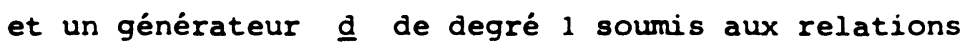


M. GROS

$$
\begin{aligned}
& \text { (3.1.1) (i) } \quad E^{a}=F a \cdot E \text { si } a \in w \Omega_{x}^{*}, E d V=d \\
& \text { (ii) } \quad a \underline{V}=\underline{V} \cdot F a \quad \text { si } a \in w \Omega_{x}^{*} \\
& \text { (iii) } \underline{v a E}=v_{a} \quad \text { si } a \in w \Omega_{X}^{*} \\
& \text { (iv) } \quad \underline{V E}=E \underline{V}=\mathbf{p} \\
& \text { (v) } \underline{d}^{2}=0 \\
& \text { (vi) } \quad \underline{d a}=d a+(-1)^{\operatorname{deg}(a)} \text { ad } \text { si } a \in \bar{w} \Omega_{x}^{*} \text {. }
\end{aligned}
$$

Un $\mathrm{R}_{\mathrm{X}}$-Module à gauche gradué est la donnée d'un w $\mathrm{g}_{\mathrm{X}}$-Module différentiel gradué muni d'opérateurs $F$ et $V$ de degré 0 et $d$ 'un opérateur a de degré 1 vérifiant le formulaire suivant

$$
\begin{array}{ll}
\text { (3.1.2) } & F V=V F=p, F d V=d, x V y=V(F x \cdot y) \text { pour tout } x \text { et } \\
& \text { tout } y, d^{2}=0, F(x y)=F x \cdot F y . \\
& d(a x)=x d a+(-1) d e g(a) a \cdot d x .
\end{array}
$$

Exemples : L'anneau $R_{X}$ lui-même, l'anneau $w \Omega_{X}(E, \underline{V}, \underline{d}$ opérant sur Wßं grâce à $F, V$ et d respectivement).

Lemme 3.1.3. Tout élément $x$ de $R_{X}^{i}$ s'écrit de manière unique sous la forme

$$
\text { (3.1.4) } \sum_{n \neq 0} a_{n} \underline{F}^{n}+\sum_{n>0} \underline{v}^{n} b_{n}+\sum_{n \neq 0} c_{n} \underline{F}^{n} d+\sum_{n>0} d \underline{v}^{n} e_{n}
$$

avec $a_{n} \cdot b_{n} \in w \delta_{X}^{i}$ et $c_{n} \cdot e_{n} \in w \Re_{X}^{i-1}$ nuls pour presque tout $n$.

La vérification est analogue à celle de l'assertion correspondante pour l'anneau $R([23, I .1 .1])$.

on posera

$$
R_{n, X}=R_{X} /\left(\underline{Y}^{n} \cdot R_{X}+d Y^{n} \cdot R_{X}\right)
$$

on prendra garde au fait que, $\left(\underline{Y}^{n} \cdot R_{X}+d Y^{n} \cdot R_{X}\right)$ n'étant pas un idéal bilatère de $R_{X}, R_{n, X} n^{\prime}$ est pas un anneau.

La projection canonique $\pi: w_{n+1} \Omega_{x} \rightarrow w_{n} \Omega_{x}$ induit un morphisme noté encore $\pi$

$$
\text { (3.1.6) } \pi: R_{n+1, x} \rightarrow R_{n, x}
$$

et faisant de la famille $R_{n, X}$ pour $n$ variable un système projectif. On posera 
Il est facile de vérifier que ce sont des anneaux et des modules à gauche sur $R_{X}$.

Avant d'énoncer la proposition suivante, définissons an morphisme canonique $\hat{R}_{\mathrm{X}} \rightarrow w \Omega_{\mathrm{X}}^{\circ}$. Il résulte de 3.1 .3 que tout élément $x$ de $R_{X}^{i}$ s'écrit de manière unique sous la forme

$$
\text { (3.1.9) } x=\sum_{n>0} a_{n} \underline{F}^{n}+\sum_{n \geqslant 0} c_{n} \underline{F}^{n} d+\sum_{n>0} \underline{v}^{n} b_{n}+\sum_{n>0} d \underline{v}^{n} e_{n}
$$

avec les deux premières sommes éventuellement infinies mais dont les coefficients $a_{n}$ et $c_{n}$ tendent vers 0 pour la topologie standard de $W \Omega_{X}$ iorsque $n$ tend vers $\infty$ et les deux dernières sommes infinies mais sans condition de convergence.

On dispose donc d'un morphisme

$(3.1 .10)$

$$
\varphi: \mathrm{R}_{\mathrm{X}}^{\wedge} \rightarrow W \Omega_{\mathrm{X}}^{\circ}
$$

défini par $\varphi(x)=\sum_{n \geqslant 0} a_{n}+\sum_{n>0} v^{n} b_{n}$.

$x$ étant supposé écrit comme dans (3.1.4).

Proposition 3.1.11. On a une suite exacte de $R_{X}$-Modules

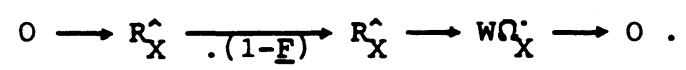

Démonstration : Soit $x \in R_{\hat{X}}^{a}$ écrit comme en (3.1.4). Dire que $x \cdot(1-\underline{E})=0$ implique

(3.1.13) $a_{0}=v b_{1}, c_{0}=p e_{1}$ et quel que soit $i \geqslant 1$

$$
a_{i}=a_{i+1}, b_{i}=v b_{i+1}, c_{i}=p c_{i-1}, e_{i}=p e_{i+1} \text {. }
$$

Comme $\bigcap_{n} p^{n} w \Omega_{x}=0$, on en déduit que $x=0$. Comme $\varphi$ est clairement surjectif, il nous reste à vérifier l'exactitude au centre de (3.1.12) i.e. que $\operatorname{Ker}(\varphi) \longrightarrow \operatorname{Im}(.(1-E))$. Or, on a

$$
\begin{aligned}
(3.1 .14) \quad x^{\prime} \cdot(1-E)= & \left(a_{0}^{\prime}-v b_{1}^{\prime}\right)+\left(c_{j}^{\prime}-p e_{1}^{\prime}\right)+\sum_{n>0}\left[\left(c_{n}^{\prime}-p c_{n-1}^{\prime}\right) \underline{E}^{n} \underline{d}+\right. \\
& \left.\underline{v}^{n}\left(b_{n}^{\prime}-b_{n+1}^{\prime}\right)+\left(e_{n}^{\prime}-p e_{n+1}^{\prime}\right) d \underline{v}^{n}+\left(a_{n}^{\prime}-a_{n-1}^{\prime}\right) \underline{E}^{n}\right] .
\end{aligned}
$$


M. GROS

Le système d'équations auquel conduit l'égalité $x^{\prime} \cdot(1-\underline{E})=x$ admet toujours une solution si $x \in \operatorname{Ker}(\varphi)$ comme on le voit facilement, d'où le résultat.

on peut de même écrire les éléments de $\widetilde{R}_{X}^{i}$ comme en (3.1) avec les sommes infinies et les coefficients tendant vers 0 pour la topologie p-adique. On a alors la

Proposition 3.1.15. On a une suite exacte de $R_{X}$-Modules (3.1.16) $0 \longrightarrow \widetilde{R}_{X} \underset{.(1-E)}{ } \widetilde{R}_{X} \rightarrow w \Omega_{X}^{\circ} \rightarrow 0$

(le morphisme $\widetilde{R}_{X} \rightarrow W \Re_{X}^{\circ}$ étant défini de la même manière que $\varphi$ ). Démonstration : C'est la même que celle de la proposition 3.1.11. Corollaire 3.1 .17 . On a une suite exacte de $R_{X} / p^{\circ}$-pro-Modules

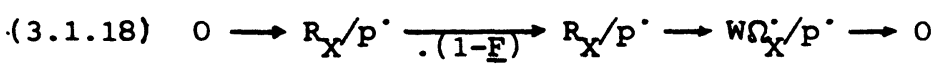

Démonstration : Il suffit de réduire modulo $p^{\bullet}$ la suite exacte précédente.

3.2. Venons-en à la définition du foncteur "points fixes sous $F$ " d'un $R_{X} / p^{\circ}$-pro-Module.

Soit M. un $R_{X} / p^{\cdot}$-pro-Module, on notera $" \underline{H o m}{ } R_{X} / p^{\cdot} \cdot\left(W \Omega_{X}^{*} / p^{\bullet}, M_{.}\right)$ le pro-w. $\Omega_{X, l o g}^{*}$-Module " $\frac{1 i m "}{j} \frac{\lim }{i} \operatorname{Hom}\left(W \Omega_{X}^{*} / p^{i}, M_{j}\right)$. Il est clair que $l$ 'on obtient bien ainsi un W. $\Omega^{*}, \log$-pro-Module puisque l'action de $F, V, d$ est $w . \Omega_{x, l o g}^{*}{ }^{-1 \text { inéaire. }}$

"Hom" ${ }_{R_{X} / p^{-}}\left(W \Omega_{X} / p^{*},-\right)$ est ainsi un foncteur exact à gauche dont le dérivé croit au sens de SGA 4, XVII, 1.2 .1 sera noté $(-)^{F}$. C'est donc un foncteur allant de $D\left(R_{X} / P^{\circ}\right.$-pro-Mod $)$ dans Ind-D (W. $\Omega_{x, \log }^{*}$ pro-Mod).

Proposition 3.2.1. Pour tout $R_{X} / p^{\cdot}$-pro-Module $M$. , on a un isomorphisme canonique

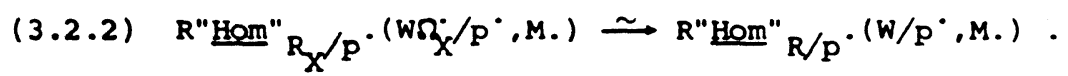




\section{COHOMOLOGIE DES FIBRÉS PROJECTIFS}

Démonstration : La suite exacte (3.1.20) fournit un isomorphisme

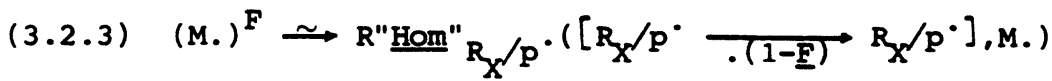

soit

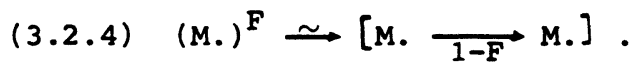

La même méthode s'applique au second membre de (3.2.2), d'où le résultat.

Corollaire 3.2 .5 . On a un isomorphisme dans Ind-D(W. $\Omega_{X}^{*} \log { }^{-p r o-M o d)}$

$$
\left(w \Omega_{\mathrm{X}}^{*} / \mathrm{p}^{*}\right)^{\mathrm{F}} \underset{\mathrm{w} . \Omega_{\mathrm{X}, \log }^{*}}{\sim}
$$

Démonstration : Il suffit d'appliquer $(3.2 .4)$ au cas $M .=w \Omega_{j}^{\circ} / p$. et d'utiliser le résultat d'Ekedahl (Lemme 9.1 de [9]) identifiant le cône de $1-F$ sur $W \Omega_{X}^{*} / p^{*}$ et sur $w . \Omega_{X}^{*}$.

Corollaire 3.2.7. Pour tout morphisme $f: X \longrightarrow Y$ entre $k$-schémas lisses et tout $R_{X} / p^{-}$-pro-Module M., on a un isomorphisme canonique $(3.2 .8)$

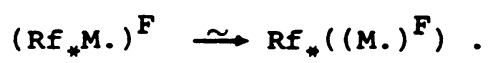

Démonstration : Grâce à (3.2.4) on a des isomorphismes

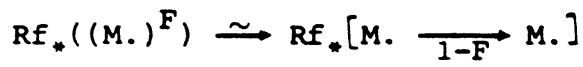

$$
\begin{aligned}
& \stackrel{\sim}{\longrightarrow}\left[\mathrm{Rf}_{*} M . \overrightarrow{I-F} \mathrm{Rf}_{*} M .\right] \\
& \sim \operatorname{Rf}_{*}\left(\left(M_{0}\right)^{F}\right) \text {. }
\end{aligned}
$$

\section{Cohomologie de Hodge-Witt des fibrés projectifs.}

4.1. On conserve les hypothèses et notations du numéro 2 . On veut calculer $R f . W S_{p}^{\circ}$ va comme objet de $D\left(X, R_{X}-\right.$ Mod).

Proposition 4.1.1. On a un isomorphisme canonique

$(4.1 .2)$

$$
\begin{aligned}
& \operatorname{Hom}_{D\left(z / p^{*}-\text { pro-Mod }\right)}\left(z / p^{*}, W \cdot R_{p}^{*}, \log [*]\right) \\
& R \\
& \operatorname{Hom}_{D\left(R^{\wedge}-\operatorname{Mod}\right)}\left(W, w \Omega_{p}[*](*)\right)
\end{aligned}
$$


M. GROS

Démonstration : Il suffit de voir que l'on a un isomorphisme $(4.1 .3)$

$$
\begin{aligned}
& \operatorname{RHom}_{z / p^{*}-\text { pro-Mod }}\left(z / p^{*}, w \cdot \Omega_{p, \log }^{*}[*]\right) \\
& R_{R^{\wedge}-\operatorname{Mod}}\left(w, w \Omega_{p}^{*}[*](*)\right) .
\end{aligned}
$$

Pour ce faire, on remarque que (3.1.12) fournit un isomorphisme

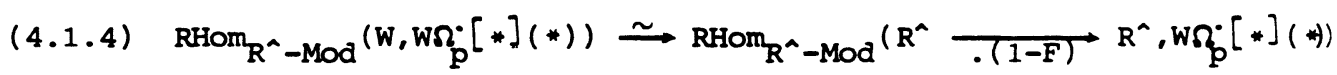

D'où des isomorphismes

$$
\begin{aligned}
& \text { (4.1.5) RHom } R^{\wedge}-\operatorname{Mod}\left(W, w \Omega_{P}^{*}[*](*)\right) \stackrel{\sim}{\sim} \Gamma\left(P_{1}\left[W \Omega_{P}^{*} \underset{1-F}{\longrightarrow} w \Omega_{P}^{*}\right][*](*)\right) \\
& \stackrel{R}{\longrightarrow} \Gamma\left(P, R \perp i m\left[W . \Omega_{P}^{*} \underset{1-F}{\longrightarrow} \text { W. } \Omega_{P}^{*}\right][*](*)\right) \\
& \stackrel{\sim}{\longrightarrow} \Gamma\left(P, R \perp i m \text { W. } \Omega_{P, \log }^{*}[*]\right) \text {. }
\end{aligned}
$$

Le dernier isomorphisme résultant de la suite exacte (1.3.2).

Le résultat découle alors de l'isomorphisme (2.1.4).

Le morphisme (2.1.6) définit donc un morphisme dans $D\left(R^{\wedge}-\right.$ Mod)

$$
c_{1}\left(\theta_{p}(1)\right): w \rightarrow w \Omega_{p}^{1}[1]
$$

et (2.1.7) joint à $(4.1 .2)$ permet de définir des morphismes

$$
c_{1}\left(\theta_{P}(1)\right)^{i}: w \rightarrow W s_{p}^{i}[i]
$$

dans $D\left(R^{\wedge}\right.$-Mod) pour tout $i \geqslant 0 \quad\left(c_{1}\left(\theta_{P}(1)\right)^{0}=I d\right)$.

On en déduit, par adjonction, un morphisme dans $D\left(R_{p}-\right.$ Mod)

(4.1.8) $\underset{i=0}{\oplus} c_{1}\left(\theta_{p}(1)\right)^{i}: \underset{i=0}{T} w \Omega_{p}(-i)[-i] \longrightarrow w \Omega_{p}$.

Utilisant d'autre part le morphisme d'adjonction

$(4.1 .9)$

$$
\text { adj : } w \Omega_{\dot{X}} \rightarrow R f_{*} w \Omega_{p}
$$

on déduit de $(4.1 .8)$ un morphisme dans $D\left(R_{X}-\right.$ Mod)

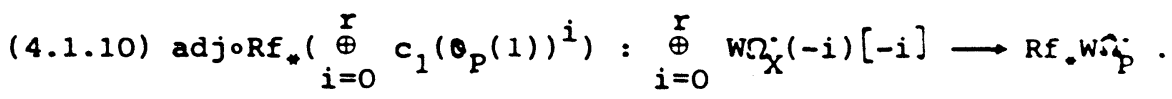


Théorème 4.1 .11 . Le morphisme $(4.1 .10)$ est un isomorphisme.

La démonstration sera donnée en 4.2 .

Corollaire 4.1.12. On a un isomorphisme canonique dans $D\left(w_{n} \Omega_{X^{-}}^{-M d g}\right)$ (4.1.13) $R_{n} \otimes_{R}^{2}(4.1 .10): \underset{i=0}{T} w_{n} \Omega_{X}(-i)[-i] \sim f_{*} W_{n} \Omega_{p}^{i}$.

Démonstration : Il suffit d'utiliser la formule de projection

$(4.1 .14) \quad R_{n} \stackrel{a}{\otimes}_{R} R f_{*} w \Omega_{p}^{\circ} \stackrel{\sim}{\sim} R f_{*}\left(R_{n} \stackrel{a}{\otimes}_{R} w \Omega_{p}^{*}\right)$

et l'isomorphisme canonique (cf. [22, I (1.2.1)])

(4.1.15) $\quad R_{n} \otimes_{R}^{u} w \Omega_{X}^{\circ} \sim w_{n} \Omega_{X}^{\circ}$

pour tout $n \geqslant 1$.

Corollaire 4.1.16. On a un isomorphisme canonique dans $\mathrm{D}\left(\mathrm{R}_{\mathrm{X}} / \mathrm{p}^{\cdot}\right.$-pro-Mod)

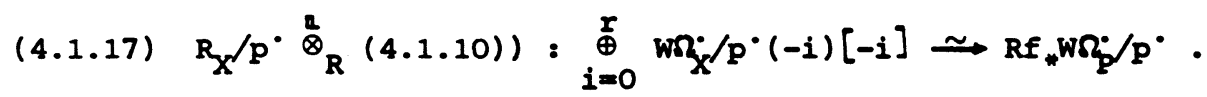

Démonstration : Il suffit d'appliquer le foncteur $/ p^{\circ}$ à (4.1.10).

Application : démonstration de 2.1.11. On applique le foncteur $(-)^{F}$ à l'isomorphisme (4.1.17). Le résultat découle alors de (3.2.6).

4.2. Démonstration de 4.1.11. Il suffit de voir que (4.1.10) est un isomorphisme dans $D(R-M O d), i . e$. que le cône $C$ de (4.1.10), considéré comme objet de $D(R-M o d)$ est nul. On va utiliser pour ce faire le lemme suivant du type "lemme de Nakayama" da à T. Ekedahl $([9])$.

Lemme 4.2.1. Soit $A$ un objet de $D^{-}(R-M o d)$. Si $R_{1}{ }_{R}^{a} A=0$, alors

$$
R_{n} \stackrel{0}{\otimes}_{R} A=0 \text { pour tout } n \geqslant 1 \text {. }
$$

Démonstration : Nous en indiquerons seulement le principe, en renvoyant d̀ [9] pour les détails.

On définit $g r_{n}$ par la suite exacte

$$
0 \longrightarrow g r_{n} \longrightarrow R_{n+1} \longrightarrow R_{n} \longrightarrow 0
$$


M. GROS

si bien que $l$ 'on est ramené à vérifier que $g r_{n} \stackrel{a}{\otimes}_{R} A=0$.

on prouve alors que $I$ 'on a une suite exacte analogue de $([21]$, I.3.9.1)

$(4.2 .3) 0 \longrightarrow R_{1}^{*} / B_{n} \longrightarrow g r_{n}^{*} \rightarrow R_{1}^{*} / z_{n}(-1) \rightarrow 0$.

Il suffit alors de prouver qu'on a

$$
B_{n} \stackrel{0}{\otimes}_{R} A^{*}=Z_{n} \otimes_{R}^{0} A=0
$$

puis par récurrence sur $n$ l'assertion analogue pour $n=1$, ce qui se voit en utilisant simultanément les deux suites exactes

$$
\text { (4.2.5) } 0 \longrightarrow \mathrm{B}_{1} \longrightarrow \mathrm{z}_{1} \stackrel{\mathrm{C}}{\longrightarrow} \mathrm{R}_{1} \longrightarrow 0
$$

où C est l'analogue de l'opération de Cartier,

$$
\text { (4.2.6) } 0 \longrightarrow \mathrm{z}_{1} \longrightarrow \mathrm{R}_{1} \stackrel{\mathrm{d}}{\longrightarrow} \mathrm{B}_{1}(1) \rightarrow 0 \text {. }
$$

Pour démontrer 4.1 .11 , il suffit donc de prouver que
$R_{1} \stackrel{a}{Q}_{R} C=0 ;$ c'est-à-dire que le morphisme

$$
\text { (4.2.7) } R_{1} \stackrel{0}{R}_{R}(4.1 .10): \underset{i=0}{T} \Omega_{X}^{(}(-i)[-i] \longrightarrow R_{*} \Omega_{p}
$$

est un isomorphisme (cf. 4.1.12).

Faute de référence, démontrons ce résultat. La question est locale sur $X$, on peut donc supposer $P$ trivial et par suite, que la suite exacte

$$
\text { (4.2.8) } 0 \longrightarrow \mathrm{f}^{*} \Omega_{\mathrm{X}}^{1} \rightarrow \Omega_{\mathrm{P}}^{1} \rightarrow \Omega_{\mathrm{P} / \mathrm{X}}^{1} \rightarrow 0
$$

est scindée et fournit donc des isomorphismes

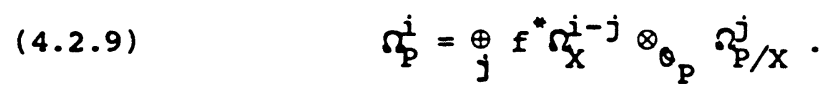

La formule de projection montre alors que $I^{\text {'on }}$ a un isomorphisme

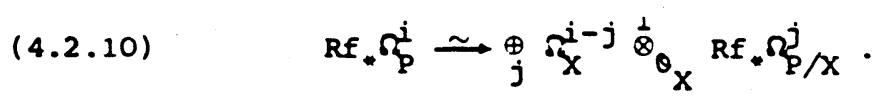

Il suffit donc de montrer que

$$
\text { (4.2.11) } \quad \operatorname{Rf}_{*} \Omega_{\mathrm{P} / \mathrm{X}} \stackrel{\sim}{\longrightarrow} \theta_{X}[-j]
$$

pour tout $j \geqslant 0$. 


\section{COHOMOLOGIE DES FIBRÉS PROJECTIFS}

Or, d'après SGA 7, exp. XI, Thm. 1.1, on a

(4.2.12) $R^{i} f_{*} R_{\mathrm{P} / \mathrm{X}}^{j}=0$ si $i \neq j$ et $R^{i} f_{*} \delta_{\mathrm{P} / \mathrm{X}}^{i} \stackrel{\sim}{\longrightarrow} \theta_{X}$.

D'où le résultat.

Corollaire 4.2.13. On a un isomorphisme de $w_{n}$-Modules compatible à $F, V$ et aux projections canoniques

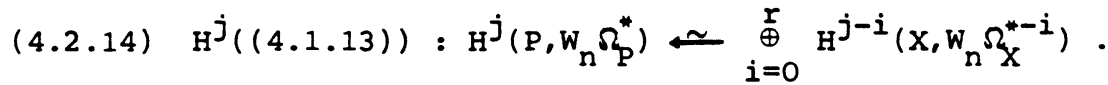

Démonstration : Il suffit d'appliquer le foncteur $\mathrm{H}^{j}(\mathrm{x},-)$ à

l'isomorphisme (4.1.13).

Corollaire 4.2.15. On a des isomorphismes de $w_{n}$-Modules

$$
\mathrm{H}^{j}\left(\mathrm{P}_{\mathrm{k}}^{\mathrm{r}}, \mathrm{w}_{\mathrm{n}} \frac{\sigma_{\mathrm{P}}^{\mathrm{r}}}{\mathrm{k}}\right)=\left\{\begin{array}{l}
0 \text { pour } i \neq j \text { ou } i>r \\
w_{n} \cdot c_{1}\left(\theta_{\mathrm{P}_{\mathrm{k}}^{r}}^{\mathrm{r}}(1)\right)^{i} \text { pour } i \in[0, r] .
\end{array}\right.
$$

Démonstration : Il suffit d'appliquer le corollaire 4.2 .13 au cas $\mathrm{X}=\operatorname{Spec}(\mathrm{k})$ et $\mathrm{E}=\mathrm{k}^{\mathrm{r+1}}$.

5. Comparaison avec la cohomologie cristalline.

5.1. Soit $u_{\mathrm{P} / \mathrm{w}_{\mathrm{n}}}$ le morphisme canonique de topos (cf. [2])

$(5.1 .1)$

$\left(\mathrm{P} / \mathrm{W}_{\mathrm{n}}\right)_{\text {cris }} \longrightarrow \mathrm{P}_{\mathrm{Zar}}$

On rappelle ([21], II Thm. 1.4) que l'on dispose d'un isomorphisme canonique fonctoriel de $D\left(w_{n}\right.$-Mod)

$(5.1 .2)$

$$
{ }^{R u_{P} / w_{n}}{ }^{\theta} \mathrm{P} / \mathrm{w}_{\mathrm{n}} \stackrel{\sim}{\longrightarrow} \mathrm{w}_{\mathrm{n}} \Omega_{\mathrm{p}}^{\dot{0}} \text {. }
$$

D'autre part, d'après $([4]$, Thm. 2.5) on dispose d'un isomorphisme canonique

$(5.1 .3)$

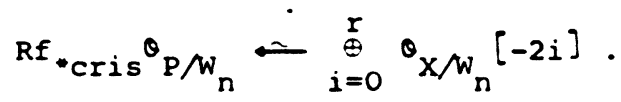

Appliquant le foncteur $R u_{X / W_{n}}$, on obtient un isomorphisme 
M. GROS

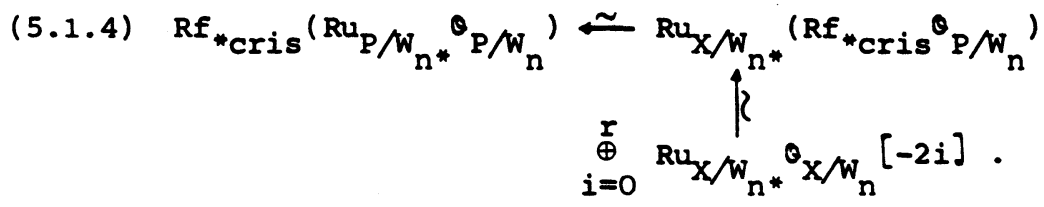

Théorème 5.1 .5 . On a un diagramme commutatif

$$
\begin{aligned}
& \text { (5.1.6) } R f_{*}\left(\operatorname{Ru}_{P / W_{n}}{ }^{\theta} P / W_{n}\right) \frac{\sim}{(5.1 .4)} \underset{i=0}{\oplus} R u_{X / W_{n}}{ }^{\theta} X / W_{n}[-2 i] \\
& \text { \} } \operatorname{Rf}_{*}(5.1 .2) \quad \text { ( }(5.1 .2) \\
& \operatorname{Rf}_{*} W_{n} \Omega_{P} \stackrel{\sim}{(4.1 .13)} \underset{i=0}{T} W_{n} \Omega_{x}^{*}(-i)[-i]
\end{aligned}
$$

Démonstration : Il suffit de vérifier la commutativité du diagramme (où $\mathrm{x}$ désigne un $\mathrm{k}$-schéma lisse quelconque)

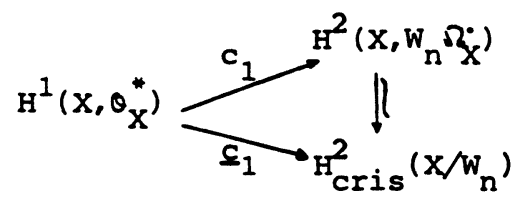

$c_{1}$ provenant de dlog $: \theta_{x}^{*} \rightarrow w_{n} \Omega_{x}^{1}, \log \rightarrow w_{n} \Omega_{x}[1]$ et $c_{1}$ étant la première classe de Chern d'un faisceau inversible telle qu'elle est définie dans ([4], (2.1.3)). Rappelons (cf. [4]) que $s_{1}$ est défini de la manière suivante. Si $J_{X / W_{n}}$ désigne l'idéal cristallin noyau du morphisme canonique $\theta_{\mathrm{x} / \mathrm{W}_{\mathrm{n}}} \longrightarrow \theta_{\mathrm{x}}$, on a une suite exacte (5.1.8) $0 \longrightarrow{ }^{1+J_{X} / w_{n}} \rightarrow \theta_{x / w_{n}}^{*} \rightarrow \theta_{x}^{*} \rightarrow 0$ d'où un morphisme $\theta_{\mathrm{x}}^{*} \longrightarrow\left(1+J_{\mathrm{X} / \mathrm{W}_{\mathrm{n}}}\right)[1]$ qui composé avec la flèche induite par le logarithme $\log : 1+J_{x / W_{n}} \rightarrow J_{x / W_{n}}$ fournit (5.1.9)

$$
c_{1}: \theta_{X}^{*} \rightarrow J_{X / W_{n}}[1]
$$

dans $D\left(X_{\text {cris }}\right)$.

Pour obtenir la commutativité de $(5.1 .7)$, il suffit donc de vérifier la commutativité du diagramme

$(5 \cdot 1.10)$

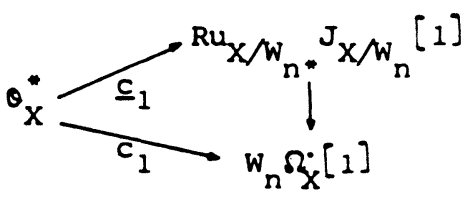


où la flèche verticale est induite par (5.1.2).

Pour ce faire, on va réaliser plus concrètement les morphismes en présence. Introduisons le complexe de De Rham multiplicatif de $I^{\prime}$ enveloppe à puissances divisées $\tilde{X}^{\prime} d^{\prime}$ un relèvement $X^{\prime}$ de $X$ sur $w_{n}$

$$
\text { (5.1.11) } \left.\Omega_{x^{\prime}}^{x}=i_{x^{\prime}}^{*} \rightarrow \Omega_{x}^{1}, \vec{d} \Omega_{x^{\prime}}^{2} \rightarrow \ldots\right) \text {. }
$$

on a un isomorphisme

$$
R u_{x / W_{n}} \theta_{x / W_{n}}^{*} \stackrel{\sim}{\longrightarrow} \Omega_{x^{*}}^{*} / w_{n}
$$

et la projection par $R u_{X / W_{n}}$ du triangle distingué déduit de (5.1.8) se déduit de la suite exacte (où I désigne l'idéal définissant $\mathrm{X}$ dans $\widetilde{\mathrm{X}}^{\prime}$ )

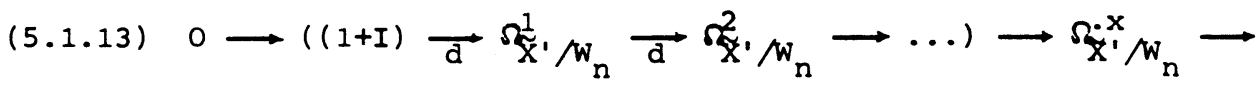

$$
\begin{aligned}
& \theta_{\mathrm{X}}^{*} \rightarrow 0
\end{aligned}
$$

On réalise ainsi sur $x_{\text {Zar }}$ le morphisme $c_{1}$ comme composé du morphisme $\theta_{\mathrm{X}}^{*} \longrightarrow\left((1+I) \underset{\mathrm{d}}{\rightarrow} \Omega_{\mathrm{X}}^{1} / \mathrm{W}_{\mathrm{n}} \longrightarrow \ldots\right)[1]$ et du morphisme induit par $\log : 1+I \longrightarrow I$.

Désignons maintenant par $w_{n} \Omega^{* x}$ le complexe de De Rham-Witt multiplicatif $: 0 \rightarrow w_{n} \theta_{x}^{*} \rightarrow w_{n} \Omega^{1} \rightarrow w_{n} \Omega_{x}^{1} \rightarrow \ldots$. on a une suite exacte

$(5.1 .14) \quad 0 \rightarrow\left(1+v w_{n-1}^{\theta} x \rightarrow w_{n} \Omega_{x}^{1} \rightarrow \cdots\right) \rightarrow w_{n} \Omega_{x}^{* x} \rightarrow \theta_{x}^{*} \rightarrow 0$.

Si maintenant on suppose donné un relèvement $\Phi$ sur $X^{\prime} d u$ Frobenius de $X^{\prime} \otimes k$, on dispose d'un morphisme $\Omega_{\dot{x}^{\prime}}^{x} / w_{n} \rightarrow w_{n} \Omega_{x}^{*}$ (cf. [21]) compatible aux morphismes de Frobenius, et s'insérant dans un diagramme commutatif envoyant la suite exacte (5.1.13) dans la suite exacte $(5.1 .14)$

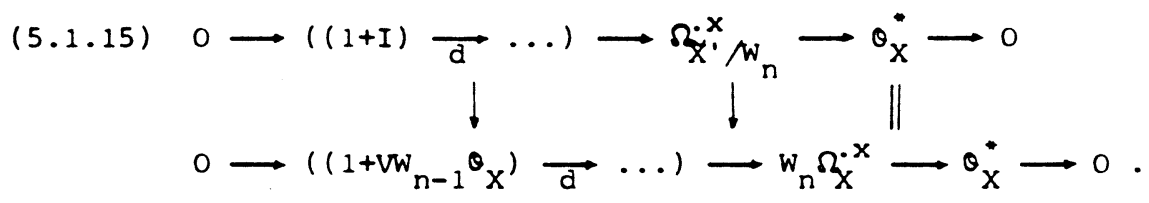


M. GROS

D'où un diagrame commutatif

$(5.1 .16)$

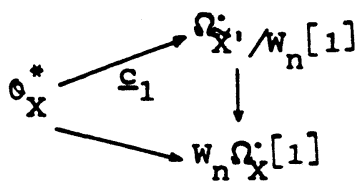

où la flèche oblique descendante est induite par la composée de la flèche $\theta_{x}^{*} \rightarrow\left(\left(1+v w_{n-1} \theta_{x}\right) \rightarrow w_{n} \Omega_{x}^{1} \rightarrow \ldots\right)[1]$ déduite de (5.1.14) et de la flèche induite par le logarithme $1+v w_{n-1}{ }^{\theta} x \rightarrow w_{n}{ }^{\theta} x$. Il reste donc à voir que cette flèche oblique descendante est bien $c_{1}$. Pour ce faire, on remarque que l'application représentant de Teichmuller $s(s(x)=\underline{x})$ de $\theta_{x}^{*}$ vers $w_{n} \theta_{x}^{*}$ permet de scinder $1 a$ suite exacte $(5.1 .14)$. Le résultat en découle. 
CHAPITRE II

MORPHISME DE GYSIN

\section{Définition des différents morphismes de Gysin.}

\subsection{Rappels sur l'autodualité du complexe de De Rham-Witt.}

Les résultats de ce numéro sont dus à $T$. Ekedahl (cf. [9]). Soit $X$ un k-schéma lisse, pour tout objet $M$ de $D_{c}^{b}\left(R_{X}\right)$ on notera $D_{X} M$ l'objet Rlim RHom $\left(M_{n}, w_{n} S_{X}^{a}[a]\right)$ de $D_{c}^{b}\left(R_{X}\right)$ (a désignant la dimension de $x$ sur $k$ ). Le premier résultat que nous aurons à utiliser est que l'on dispose d'un isomorphisme canonique dans $D_{c}^{b}\left(R_{X}\right)$

$$
w \Omega_{X}^{*} \stackrel{\sim}{\longrightarrow}\left(D_{X} w \Omega_{X}^{*}\right)(-a)[-a]
$$

Le second résultat est le suivant : soit $f: Y \longrightarrow X$ un morphisme propre de $k$-schémas lisses et $M$ un objet de $D_{C}^{b}\left(R_{Y}\right)$, on peut alors construire un isomorphisme de dualité

$$
R f_{*} D_{Y} M \stackrel{\sim}{\longrightarrow} D_{X} R f_{*} M
$$

obtenu en composant l'applicacion canonique $\operatorname{Rf}_{*} D_{Y} M \longrightarrow$ $\operatorname{Rlim} \operatorname{RHom}\left(R f_{*} M_{n}, R f_{*} W_{n} R_{Y}^{b}[b]\right.$ ) (où $b=\operatorname{dim}_{k}(Y)$ ) et une application

$$
R_{*} w_{n}{ }^{b}[b] \rightarrow w_{n}{ }^{a}[a]
$$

appelé trace et obtenu en utilisant le fait que $w_{n} \Omega_{x}^{a}$ est dualisant.

\subsection{Définitions.}

On va définir pour tout morphisme propre $f: Y \rightarrow X$ entre $k$-schémas lisses $(\operatorname{dim}(Y)=b, \operatorname{dim}(X)=a)$ comme ci-dessus des morphismes de Gysin pour divers faisceaux. On posera $r=a-b$.

Définition 1.2.1. Le morphisme de $D_{c}^{b}\left(R_{X}\right)$

$$
f_{*}: \operatorname{Rf}_{*} w \Omega_{\dot{Y}} \longrightarrow w \Omega_{\dot{X}}(r)[r]
$$


M. GROS

est le transposé (par autodualité des complexes en présence) du morphisme $f^{*}:$ WR: $\rightarrow R_{*} W \Omega_{i}$.

Détaillons un peu cette définition. (1.1.1) induit un isomorphisme de $D_{c}^{b}\left(R_{X}\right)$

$$
\operatorname{Rf}_{*} W S_{Y} \longrightarrow \operatorname{Rf}_{*} D_{Y} W \Omega_{Y}^{*}(-b)[-b] .
$$

D'où grâce à $(1.1 .3)$ un morphisme

$$
R f_{*} W \Omega_{Y}^{*} \longrightarrow D_{X} R f_{*} W \Omega_{Y}^{*}(-b)[-b] \text {. }
$$

$D_{X}$ étant contravariant, $(1.1 .1)$ montre que $f^{*}: w \Omega_{X}^{*} \rightarrow R f_{*} w \Omega_{Y}^{*}$ induit un morphisme noté $f_{*}$

$$
\operatorname{Rf}_{*} W \Omega_{Y} \rightarrow W \Omega_{X}(r)[r] .
$$

Définition 1.2.6. On notera encore $f_{*}: R f_{*} W \Omega_{Y^{*}} / p^{*} \rightarrow W \Omega_{X^{*}} / P^{*}(r)[r]$ le morphisme de $D\left(R_{X} / p^{\cdot}\right.$-pro-Mod $)$ défini par $\left.R_{X} / p^{\cdot} \stackrel{0}{\otimes}_{R_{X}}(1.2 .5)\right)$.

Définition 1.2.7. On notera encore $f_{*}: R f_{*} W . \Omega_{Y, \log }^{*} \rightarrow W . S_{X}^{*}+r \log [r]$ le morphisme de $D\left(W . \Omega_{X, l o g}^{*} \text {-pro-Mod) défini par }(1.2 .6)\right)^{F}$.

Définition 1.2.8. On notera encore $f_{*}: R f_{*} W_{n} \Omega_{Y, \log }^{*} \rightarrow W_{n} \Omega_{X, \log }^{*}[r]$ le morphisme de $D\left(W_{n} \Omega_{x, l o g}^{*}{ }^{-M o d}\right)$ défini par $z / p^{n} \otimes(1.2 .7)$, compte tenu de I 1.3.4.

\section{Propriétés.}

On va maintenant énoncer quelques propriétés du morphisme de Gysin de 1.2.8 qui est, en un sens, le "plus fin" des quatre. Toutes ces propriétés resteront valables, mutatis mutandis, pour les morphismes de Gysin 1.2.1, 1.2 .6 et 1.2.7.

\subsection{Transitivité.}

Considérons deux morphismes propres $\mathrm{E}: \mathrm{Y} \longrightarrow \mathrm{X}$ et $\mathrm{g}: \mathrm{Z} \longrightarrow \mathrm{Y}$ entre $k$-schémas lisses. Alors 
Proposition 2.1.1. On a

(2.1.2) $(f g)_{*}=f_{*} \circ R f_{*}\left(g_{*}\right): R(f g)_{*} W_{n} \Omega_{z, \log } \longrightarrow w_{n} \Omega_{x, l o g}^{*+r+r^{\prime}}\left[r+r^{\prime}\right]$.

Démonstration : Par construction des morphismes en présence, il suffit de prouver l'assertion analogue pour les morphismes du type $(1.2 .2)$, c'est-à-dire sur $w_{n} \Omega_{X}^{\circ}$ ce qui résulte immédiatement de la formule

$(2.1 .3)(f g)^{*}=R f_{*}\left(g^{*}\right) \circ f^{*}: W_{n} \Omega_{X}^{*} \longrightarrow R(f g)_{*} W_{n} \Omega_{z}^{*} \cdot$

\subsection{Formule de projection.}

Proposition 2.2.1. Soit $f$ un morphisme propre comme en 1.2 , on a un diagrame commutatif

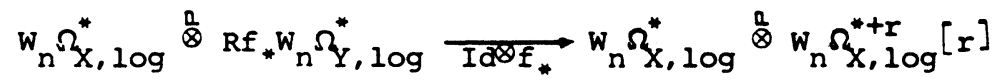

(2.2.2) $\quad f^{*} \otimes I d$

$\left\lceil\gamma_{x}\right.$

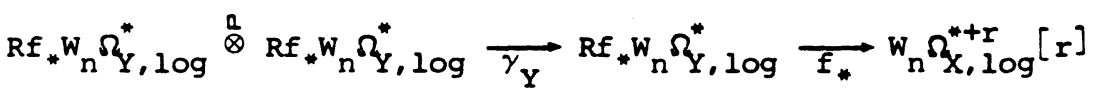

avec $\gamma_{\mathrm{X}}$ (resp. $\gamma_{\mathrm{Y}}$ ) le morphisme de produit et $\otimes=\otimes_{\mathrm{W}_{\mathrm{n}} \Omega_{\mathrm{X}, \mathrm{log}}^{*}}$

Démonstration : On décompose le diagrame (2.2.2) en deux parties. on a un diagramme commutatif

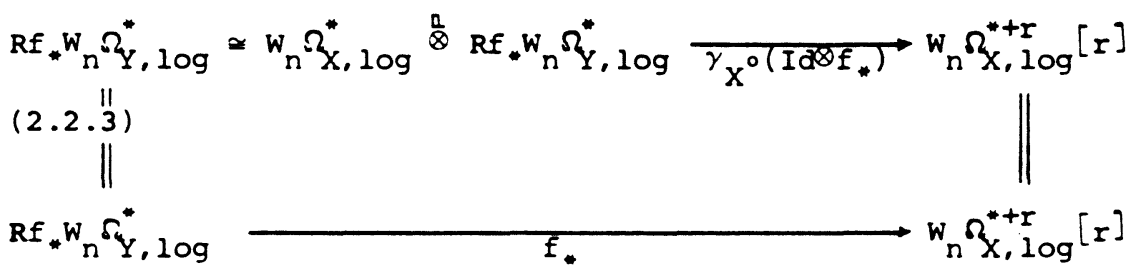

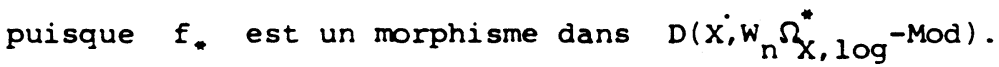

D'autre part, on a un diagrame commutatif évident

(2.2.4) $w_{n} \Omega_{X, 10 g}^{*}$ Rf. $W_{n} \Omega_{Y, l o g}^{*}$

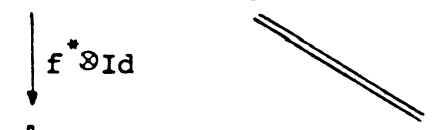

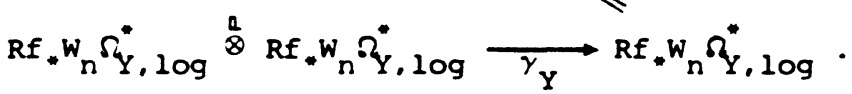


M. GROS

D'où le résultat.

Corollaire 2.2.5. Pour tout $y \in H^{*}\left(Y, w_{n} \Omega_{Y, l o g}^{*}\right)$ et tout $x \in$ $H^{*-r}\left(X, w_{n} \Omega^{*-r}, \log ^{\prime}\right)$, on a

(2.2.6) $f_{*}\left(y \cdot f^{*}(x)\right)=f_{*}(y) \cdot x$ dans $H^{*}\left(x, w_{n} \Omega_{x, l o g}^{*}\right)$

où $f_{*}$ désigne le morphisme induit en cohomologie

$H^{*-r}\left(X, W_{n} \Omega_{Y, l o g}^{*-r}\right) \longrightarrow H^{*}\left(X, W_{n} \Omega_{X, l o g}^{*}\right)$ par $f_{*}$ de la définition 1.2.8.

Démonstration : On applique le foncteur $R \Gamma(X,-)$ au diagrame commutatif (2.2.2) puis on en prend la cohomologie, (2.2.6) est alors la traduction de la commutativité du diagramme que l'on obtient.

Définition 2.2.7. Si $f$ est une immersion fermée, l'élément $c l(Y / X):=f_{*}\left(1_{Y}\right) \in H^{Y}\left(X, W_{n} \Omega_{X, l o g}^{x}\right)$ sera appelé classe de cohomologie de $Y$ dans $X$.

Supposons dans la fin de ce numéro que $f$ est une immersion fermée.

Corollaire 2.2.8. Le morphisme $f_{*} f^{*}: R \Gamma\left(x, w_{n} \Omega_{x, l o g}^{*}\right) \rightarrow$ $R \Gamma\left(X, W_{n} \Omega_{X, l o g}^{*+r}\right)[r]$ est le produit par $c l(Y / X)$.

Démonstration : On remarque simplement que le produit avec $c l(Y / X)$ est le morphisme

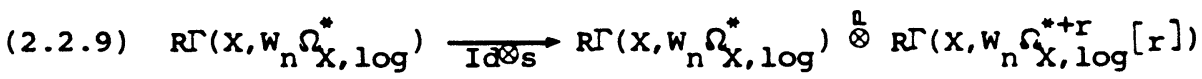

$$
\begin{gathered}
\gamma_{X} \\
R \Gamma\left(X, W_{n} \Omega_{x, \log }^{*+r}\right)[r]
\end{gathered}
$$

où $s$ est le morphisme

$$
z / p^{n} \longrightarrow R \Gamma\left(Y, W_{n} \Omega_{Y, \log }^{*}\right) \underset{f_{*}}{\longrightarrow} R \Gamma\left(Y, W_{n} \Omega_{Y, l o g}^{*+r}\right)[r] .
$$

Le corollaire découle alors de la commutativité de (2.2.2).

Proposition 2.2.10. Pour tout $y \in H^{*}\left(Y, W_{n} \Omega_{Y, \log }^{*}\right)$, on a

$$
(2.2 .11) f^{*} f_{*}(y)=y \cdot f^{*} f_{*}(1) \quad \text { dans } H^{*+r}\left(Y, W_{n} R^{*+1}, \operatorname{rog}\right) \text {. }
$$


Démonstration : Elle sera donnée au chapitre IV en même temps que nous calculerons $f^{*} f_{*}(1)$ (cf. IV (2.1.2)).

\subsection{Transversalité.}

Considérons un diagramme cartésien

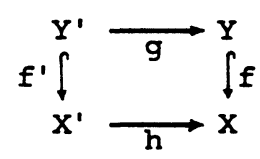

où tous les schémas sont des k-schémas lisses et où $f$ et $f^{\prime}$ sont des immersions fermées. On a alors la

Proposition 2.3.2. Si $f$ et $h$ sont transverses, on a (2.3.3) $R h_{*}\left(f_{*}^{\prime}\right) R f_{*}\left(g^{*}\right)=h^{*} f_{*}: R f_{*} W_{n} \Omega_{Y, \log }^{*} \rightarrow R h_{*} W_{n} \Omega_{X^{\prime}, \log }^{*+r}[r]$. Démonstration : La démonstration sera donnée au numéro 3 .

Corollaire 2.3.4. On a

$(2.3 .5) \quad h^{*} c l(Y / X)=c l\left(Y^{\prime} / X^{\prime}\right) \quad$ dans $H^{r}\left(X^{\prime}, w_{n} \Omega^{r} X^{\prime}, \log { }^{\prime}\right.$.

\subsection{Formule d'intersection.}

Proposition 2.4.1. Soient $f: Y \rightarrow X$ et $f^{\prime}: Y^{\prime} \rightarrow X$ deux immersions fermées lisses de codimension respectivement $r$ et $r^{\prime}$. On pose $T=Y X_{X} Y^{\prime}$. Supposons que $Y$ et $Y^{\prime}$ se coupent transversalement, on a alors

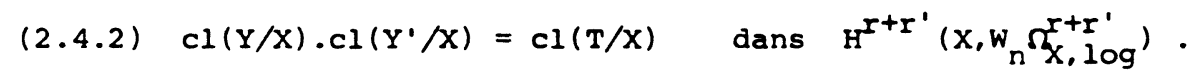

Démonstration : On a par définition $c l(Y / X)=f_{*}(1), c l\left(Y^{\prime} / X\right)=f_{*}^{\prime}(1)$. La formule de projection 2.2.6 donne

$(2.4 .3) \quad f:(1) . f_{*}(1)=f:\left(1 . f^{*} f_{*}(1)\right)$.

Soit $f^{\prime \prime}$ (resp. $f^{\prime \prime \prime)}: T \longrightarrow Y$ (resp. $: T \longrightarrow Y^{\prime}$ ) la première (resp. seconde) projection, $Y$ et $Y^{\prime}$ se coupant transversalement, $(2.3 .5)$ donne

(2.4.4) $f^{\prime \prime} f_{*}(1)=f^{\prime \prime} \operatorname{cl}(Y / X)=\operatorname{cl}\left(T / Y^{\prime}\right)=f_{* \prime \prime}^{\prime \prime}(1)$ 
M. GROS

d ' où

$(2.4 .5) \quad f_{*}^{\prime}(1) \cdot f_{*}(1)=f_{*}^{\prime \prime \prime \prime}(1)$

d'où, grâce à $(2.1 .2)$

$(2.4 .6) \quad f_{*}^{\prime}(1) \cdot f_{*}(1)=\left(f^{\prime} f^{\prime \prime \prime}\right)_{*}(1)=c l(T / X)$.

Remarque. On prouvera au numéro 4.2 une formule plus générale.

\subsection{Classe de cycle et classe de Chern.}

Proposition 2.5.1. Soit $f$ une immersion fermée de codimension 1 définie par un idéal inversible $J$. On a

$$
\text { (2.5.2) } f_{*}(1)=c l(Y / X)=-c_{1}(J) \text { dans } H^{1}\left(X, w_{n} \Omega^{1}, \log \right) \text {. }
$$

Démonstration : Elle sera donnée au numéro 3 .

\subsection{Cas d'un morphisme $P(E) \longrightarrow X$.}

Soit toujours $x$ un $k$-schéma lisse. Soit $E$ un $\theta_{x}$-Module localement libre de rang $r^{\prime}+1$. On notera $f$ la projection $Y=P(E) \longrightarrow X$. $c^{\prime}$ est un morphisme propre et $r=-r^{\prime}$. On a la

Proposition 2.6.1. On a

(2.6.2) $f_{*} \circ c_{1}\left(\theta_{Y}(1)\right)^{r^{\prime}}=I d: w_{n} \Omega_{X, \log }^{*} \rightarrow w_{n} \Omega_{X, \log }^{*}$

où $c_{1}\left(\theta_{Y}(1)\right)^{Y^{\prime}}$ est le morphisme intervenant dans $I(2.1 .10)$.

Démonstration : La question est locale sur $x$, on peut donc supposer que $f$ admet une section $i$. On a donc un diagrame commutatif

$$
\text { Id }\left.\right|_{X} ^{I / f}
$$

on en déduit que $f_{*} i_{*}=I d$ sur $w_{n} \Omega_{x, l o g}$.

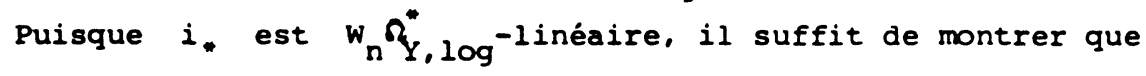

(2.6.4) $c_{1}\left(\theta_{Y}(1)\right)^{Y^{\prime}}=i_{.}(1)$ dans $H^{Y^{\prime}}\left(Y, W_{n} R^{Y^{\prime}}, \log \right)^{\prime}$. 
Itérant la formule d'intersection (2.4.2) $r$ ' fois, on en déduit que la classe de cohomologie de l'intersection de $r$ ' hyperplans est le produit $I^{\prime}$ fois de la classe de cohomologie d'un hyperplan, classe égale à $c_{1}\left(\theta_{Y}(1)\right)$. D'où

$$
i_{*}(1)=c_{1}\left(\theta_{Y}(1)\right)^{Y^{\prime}} \text {. }
$$

\subsection{Cas d'un morphisme birationnel.}

Supposons que le morphisme propre $f: Y \rightarrow X$ soit birationnel, on a alors la

Proposition 2.7.1. $f_{*}$ oadj : $w_{n} \Omega_{x, l o g}^{*} \longrightarrow R f_{*} w_{n} \Omega_{Y, l o g}^{*} \longrightarrow w_{n} \Omega_{x, l o g}^{*}$ est égal au morphisme identité.

Démonstration : On remarque simplement que le morphisme composé

$$
\begin{aligned}
& f_{*} f^{*}: w_{n} \Omega_{x, \log }^{*} \longrightarrow R f_{*} f^{*} w_{n} \Omega_{x, \log }^{*} \rightarrow R f_{*} W_{n} \Omega_{Y, \log }^{*} \\
& w_{n} \Omega_{x, ~}^{*} \log
\end{aligned}
$$

est l'identité car il coIncide avec l'identité sur un ouvert dense (f étant birationnel). D'où le résultat.

Corollaire 2.7.3. Sous 1'hypothèse f birationnel,

(2.7.4) $f^{*}: H^{*}\left(x, w_{n} \Omega_{X, l o g}^{*}\right) \rightarrow H^{*}\left(Y, w_{n} \Omega_{Y, 1 \circ g}^{*}\right)$

est injective.

\section{Cas d'une immersion fermée.}

3.1. Préliminaires d'alqèbre homoloqique.

Soit $A^{*}$ un complexe borné $d^{\prime}$ une catégorie abélienne possédant suffisamment d'injectifs dont la différentielle sera notée $d$. On pose 
M. GROS

$$
\begin{aligned}
\mathrm{ZA}^{j} & =\operatorname{Ker}\left(d: A^{j} \longrightarrow A^{j+1}\right) \\
\mathrm{BA}^{j} & =\operatorname{Im}\left(d: A^{j-1} \longrightarrow A^{j}\right) \\
\mathrm{H}^{j}\left(A^{j}\right) & =\mathrm{ZA}^{j} / B A^{j} .
\end{aligned}
$$

Soit d'autre part $G$ un foncteur additif exact à gauche allant de la catégorie abélienne ci-dessus dans elle-même.

Considérons l'énoncé suivant

(3.1.2) Il existe un entier $r$ tel que pour tout $j$ on ait $R^{i} G\left(A^{j}\right)=R^{i} G\left(\underline{H}^{j}\left(A^{\cdot}\right)\right)=0$ pour $i \neq r$ et $R^{i} G\left(Z A^{j}\right)=R^{i} G\left(B A^{j}\right)=0$ pour $i$ suffisamment grand.

Lemme 3.1.3. Supposons (3.1.2) vérifié, alors

(3.1.4) $R^{i} G\left(Z A^{j}\right)=R^{i} G\left(B A^{j}\right)=0$ pour tout $j$ et tout $i \neq r$.

Démonstration : On a deux suites exactes

$$
\begin{aligned}
& 0 \longrightarrow \mathrm{BA}^{j} \longrightarrow \mathrm{ZA} \mathrm{A}^{j} \longrightarrow \mathrm{H}^{j}\left(\mathrm{~A}^{\circ}\right) \longrightarrow 0 \\
& \mathrm{O} \longrightarrow \mathrm{ZA} \mathrm{A}^{j} \longrightarrow \mathrm{A}^{j} \longrightarrow \mathrm{BA}^{j+1} \longrightarrow 0 .
\end{aligned}
$$

On écrit alors les deux suites exactes longues de cohomologie du foncteur $G$ qui en résultent et l'on déduit (3.1.4) par récurrence ascendante sur $i$ pour $i<r$ et récurrence descendante sur $i$ pour $i>r$.

Posons maintenant

$$
\begin{aligned}
& \text { (3.1.7) } z^{j} R^{i} G\left(A^{*}\right)=\operatorname{Ker}\left(R^{i} G(d): R^{i} G\left(A^{j}\right) \longrightarrow R^{i} G\left(A^{j+1}\right)\right) \\
& { }_{B}{ }^{j} R^{i} G\left(A^{*}\right)=\operatorname{Im}\left(R^{i} G(d): R^{i} G\left(A^{j-1}\right) \longrightarrow R^{i} G\left(A^{j}\right)\right) \\
& \underline{H}^{j}\left(R^{i} G\left(A^{j}\right)\right)=z^{j} R^{i} G\left(A^{*}\right) / B^{j} R^{i} G\left(A^{*}\right) \text {. }
\end{aligned}
$$

$L^{\prime}$ injection canonique $\mathrm{ZA}^{j} \longrightarrow \mathrm{A}^{j}$ donne un morphisme canonique (3.1.8) $R^{i} G\left(2 A^{j}\right) \longrightarrow z^{j} R^{i} G\left(A^{*}\right)$.

Supposons maintenant (3.1.2) vérifié, (3.1.6) induit une suite exacte

$$
\text { (3.1.9) } \left.0 \longrightarrow R^{r} G\left(Z A^{j}\right) \longrightarrow R_{34}^{R_{G}} A^{j}\right) \longrightarrow R^{r} G\left(B A^{j+1}\right) \longrightarrow 0 .
$$


Par définition, on a une suite exacte

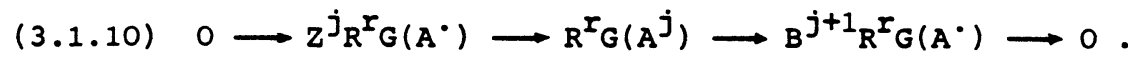

On déduit donc de (3.1.8) un morphisme

(3.1.11) $R^{r} G\left(B A^{j+1}\right) \longrightarrow B^{j+1} R^{r} G\left(A^{*}\right)$.

Proposition 3.1.12. Supposons (3.1.2) vérifié, (3.1.8) et $(3.1 .11)$ sont alors des isomorphismes fonctoriels pour tout $j$.

Démonstration : Pour (3.1.8), si $i \neq r$, les deux membres sont nuls. Par définition de (3.1.11), l'assertion relative aux bords est équivalente à celle concernant les cycles. Prouvons cette dernière. Par définition, on a

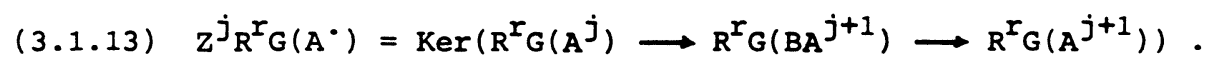

Prouvons que $R^{r} G\left(B A{ }^{j+1}\right) \longrightarrow R^{r} G\left(A^{j+1}\right)$ est une injection, la proposition 3.1 .12 en résultera grâce à la suite exacte (3.1.9). $L^{\prime}$ injection $B A^{j+1} \longrightarrow A^{j+1}$ se factorise en les deux injections $\mathrm{BA}^{\mathrm{j}+1} \longrightarrow \mathrm{ZA}^{\mathrm{j}+1}$ et $\mathrm{ZA}^{\mathrm{j}+1} \longrightarrow \mathrm{A}^{\mathrm{j}+1}$. Or 3.1 .3 appliqué aux suites exactes $(3.1 .5)$ et $(3.1 .6)$ montre que 1 'on a des injections

$$
\begin{aligned}
& (3.1 .14) \quad R^{r} G\left(B A^{j+1}\right) \longrightarrow R^{r} G\left(Z A^{j+1}\right) \\
& (3.1 .15) \quad R^{r} G\left(Z A^{j+1}\right) \longrightarrow R^{r} G\left(A^{j+1}\right),
\end{aligned}
$$

d'où une injection

$$
\text { (3.1.16) } R^{r} G\left(B A^{j+1}\right) \smile R^{r} G\left(A^{j+1}\right) \text {. }
$$

d'où la conclusion. On déduit de (3.1.5) une suite exacte

$$
(3.1 .17) 0 \longrightarrow R^{r} G\left(B A^{j}\right) \longrightarrow R^{r} G\left(Z A^{j}\right) \longrightarrow R^{r} G\left(\underline{H}^{j}\left(A^{\cdot}\right)\right) \longrightarrow 0 .
$$

D'autre part, on a par définition une suite exacte

$(3.1 .18) \quad 0 \longrightarrow B^{j} R^{I} G\left(A^{*}\right) \longrightarrow z^{j} R^{I} G\left(A^{*}\right) \longrightarrow \underline{H}^{j}\left(R^{I} G\left(A^{*}\right)\right) \longrightarrow 0$.

Les morphismes $(3.1 .8)$ et $(2.1 .11)$ inciuisent donc un morphisme

$$
\text { (3.1.19) } \quad R^{\Gamma} G\left(\underline{H}^{j}\left(A^{*}\right)\right) \longrightarrow \underline{H}^{j}\left(R^{r} G\left(A^{*}\right)\right)
$$

et 3.1 .12 implique 
Corollaire 3.1.20. Le morphisme (3.1.19) est un isomorphisme fonctoriel pour tout $j$.

Remarque . Comme le fait remarquer Gabber, on peut aussi obtenir (3.1.19) de la façon suivante. On considère les deux suites spectrales

$$
\begin{aligned}
& \text { (3.1.21) } E_{1}^{i j}=R^{j} G\left(A^{i}\right) \Rightarrow R^{*} G\left(\underline{B} A^{*}\right) \\
& (3.1 .22) \quad E_{2}^{i j}=R^{i} G\left(\underline{H}^{j}\left(A^{*}\right)\right) \Longrightarrow R^{*} G\left(\underline{s} A^{*}\right) .
\end{aligned}
$$

Les hypothèses $(3.1 .2)$ impliquent qu'elles dégénèrent toutes deux en $E_{2}$ et fournissent donc des isomorphismes

$$
\text { (3.1.23) } R^{r} G\left(\underline{H}^{j-r}\left(A^{*}\right)\right) \stackrel{\sim}{\sim} R^{j} G\left(\underline{B A} A^{*} \underset{H^{j-r}}{\sim}\left(R^{r} G\left(A^{*}\right)\right)\right. \text {. }
$$

\subsection{Construction locale du morphisme de Gysin.}

L'objet de ce numéro est de donner une construction du morphisme de Gysin attaché à une immersion fermé à l'aide de relèvements locaux. On vérifiera plus tard la colncidence avec la définition de 1.2 .

Nous noterons $I_{Y}(-)$ le foncteur "sections locales à support dans $Y^{\prime \prime}$ ( $Y$ désignant un sous-schéma fermé lisse de $X$ de codimension $r$ ). Pour tout complexe de $\mathbf{z}$-Modules $A^{\circ}$, on pose

$$
\text { (3.2.1) } \quad \Gamma_{Y}^{j}\left(A^{*}\right)=I_{Y}\left(A^{j}\right) \text {. }
$$

On a donc un foncteur exact à gauche $\Gamma_{Y}$ de la catégorie des complexes de Z-Modules dans elle-même dont les dérivés droits seront notés $\underline{H}_{Y}^{* m}(-)$. Il est clair que $l^{\prime}$ on a des isomorphismes canoniques

$$
\text { (3.2.2) } \quad H_{Y}^{q \cdot m}\left(A^{*}\right) \stackrel{\sim}{\longrightarrow} \mathbb{E}_{Y}^{m}\left(A^{q}\right) .
$$

On va définir un morphisme de complexes de z-Modules

$$
\text { f. : } W_{n} \Omega_{Y}(-r) \rightarrow H_{Y}^{* x}\left(w_{n} \Omega_{X}^{*}\right) \text {. }
$$

On commencera par définir un morphisme de modules gradués

$$
f_{*}: w_{n} \Omega_{Y}^{*}(-r) \longrightarrow H_{Y}^{* r}\left(w_{n} \Omega_{X}^{*}\right) \text {. }
$$


Supposons tout d'abord que $f$ se relève en une immersion fermée $f^{\prime}: Y^{\prime} \longrightarrow X^{\prime}$ de $W_{n}$-schémas lisses.

On dispose dans cette situation, du morphisme de Gysin de $f^{\prime}$ $([2]$, VI $(3.1 .1))$

$$
\text { (3.2.5) } f_{*}^{\prime}: \Omega_{Y}^{*} / W_{n}(-x) \rightarrow E_{Y}^{\prime I}\left(\Omega_{X}^{*} / W_{n}\right) \text {. }
$$

Ce morphisme induit en cohomologie un morphisme

$$
\text { (3.2.6) } \left.\underline{\underline{H}}\left(f_{*}^{\prime}\right): \underline{H}^{*}-r_{(} \Omega_{Y}^{*} / W_{n}\right) \rightarrow \underline{H}^{*}\left(\underline{H}_{Y}^{*}\left(\Omega_{X^{\prime}}^{*} / W_{n}\right)\right) .
$$

On va maintenant appliquer le corollaire 3.1 .20 au second membre de (3.2.6). Il faut pour ce faire, vérifier que l'on a bien (3.1.2). Ceci pourrait être vu en utilisant les liens existant entre $\underline{H}^{*}\left(\Omega_{X}^{*} / w_{n}\right)$ et $w_{n} \Omega_{X}^{*}$ mais $l^{\prime}$ on va prouver un résultat plus fort.

on remarque que $I^{\prime}$ on peut considérer $z \Omega_{X^{\prime}}^{j} / W_{n}, B \Omega^{j} / W_{n}$, $\underline{H}^{j}\left(\Omega_{X^{\prime} / W_{n}}\right)$ comme des modules sur $l^{\prime}$ anneau $\underline{H}^{0}\left(\Omega_{X^{\prime}}^{\circ} / W_{n}\right)\left(\sim w_{n} \theta_{X}\right)$.

Considérons alors la filtration p-adique sur les trois modules précédents et posons $\mathrm{gr}^{i}=\mathrm{Fil}^{i} / \mathrm{Fil}^{i+1}$, il est clair que chacun des trois modules $g r^{i} z \Omega_{X^{\prime} / w_{n}}^{j}, g r^{i} B \Omega_{X^{\prime} / w_{n}}^{j}, g r^{i} \underline{H}^{j}\left(\Omega_{X^{\prime}} / w_{n}\right)$ est muni $d^{\prime}$ une structure de $\theta_{X}^{\left(p^{n}\right)}$-Module. On a alors la

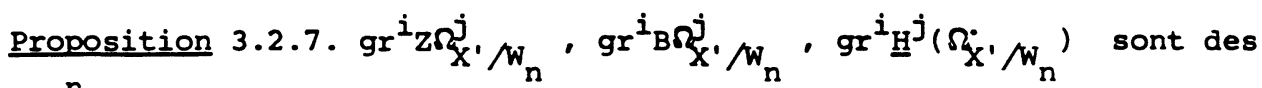
$\theta_{X}^{\left(p^{n}\right)}$-Modules localement libres de type fini.

Démonstration : La méthode est standard (cf. $[22,0.2 .2 .8]$ et [26]) : chacun des trois faisceaux étant compatible à la localisation étale, on peut supposer que $x^{\prime}=\operatorname{spec}\left(w_{n}\left[t_{1}, \ldots, t_{a}\right]\right)$ avec $a=\operatorname{dim}(X)$.

Soit alors $\bar{X}^{\prime}$ le schéma déduit de $X^{\prime}$ par extension des scalaires à une clôture algébrique de $k$. Les images inverses des trois faisceaux sur $\bar{x}^{\prime}$ s'identifient à $g r^{i} z \Omega_{X^{\prime}}^{j} / w_{n}, g r^{i} B \Omega_{X^{\prime}}^{j} / w_{n}$. $g^{i} \underline{H}^{j}\left(\Omega_{X^{\prime}} / W_{n}\right)$ et sont localement libres sur un ouvert non vide de $\overline{\mathrm{x}}^{\prime}$ car cohérents.

Comme ces trois faisceaux sont invariants par translation, des translations par des $k$-points de $\bar{x}^{\prime}$ montrent qu'ils sont partout localement libres de iype fini. 
Corollaire 3.2 .8 . On a

(3.2.9) $\quad H_{Y}^{i},\left(Z \Omega_{X}^{j}, W_{n}\right)=H_{Y}^{i},\left(B \xi_{X^{\prime}}^{j} / W_{n}\right)=H_{Y}^{i},\left(H^{j}\left(\Omega_{X}^{j} / W_{n}\right)\right)=0$

$$
\text { pour tout } i \neq x \text {. }
$$

Démonstration : On filtre les trois modules par la filtration p-adique. Comme les gradués correspondants sont localements libres, on en déduit le résultat voulu, $Y$ étant de profondeur $r$.

on a donc un isomorphisme canonique

$$
\underline{H}^{*}\left(\underline{H}_{Y}^{\cdot} \cdot r\left(\Omega_{X}^{*} / w_{n}\right)\right) \stackrel{\sim}{\longrightarrow} \cdot H_{Y}^{\prime}\left(\underline{H}^{*}\left(\Omega_{X}^{*} / w_{n}\right)\right),
$$

et (3.2.6) s'interprète donc comme un morphisme

$$
\begin{aligned}
& \left.(3.2 .11) \quad \underline{H}^{*}\left(f_{*}\right): \underline{H}^{*}-\Omega_{Y^{\prime} / W_{n}}\right) \longrightarrow \underline{H}_{Y^{\prime}}^{*}\left(\underline{H}^{*}\left(\Omega_{X^{\prime} / W_{n}}\right)\right) . \\
& \text { Comme on a un isomorphisme }([20], \operatorname{III~}(1.5 .2))
\end{aligned}
$$

(3.2.12) $\underline{\mathrm{H}}^{*}\left(\Omega_{\mathrm{X}}^{*} / \mathrm{w}_{\mathrm{n}}\right) \stackrel{\sim}{\longrightarrow} \mathrm{w}_{n} \Omega_{\mathrm{X}}^{*}$.

on déduit de $(3.2 .11)$ un morphisme que l'on notera $f_{*}$

$$
(3.2 .13) \quad f_{*}: W_{n} \Omega_{Y}^{*}(-I) \longrightarrow H_{Y}^{*}\left(W_{n} \Omega_{X}^{*}\right) \text {. }
$$

Pour terminer la construction de (3.2.4), on remarque que la construction précédente est indépendante du relèvement $f^{\prime}$ de $f$ choisi (on emploie le même argument que pour prouver que le premier membre de (3.2.12) est indépendant du relèvement $X^{\prime}$ de $X$ choisi). D'autre part, si $f$ ne se relève pas en une immersion fermée $f^{\prime}$, on peut toujours trouver un recouvrement de $X$ par des ouverts $U_{i}$ tels que $f \mid U_{i}$ se relève en une immersion fermée de $w_{n}$-schémas comme ci-dessus. On vérifie alors que les divers morphismes $f_{*} \mid U_{i}$ se recollent en un morphisme $f$, comme annoncé.

\subsection{Description du morphisme de Gysin.}

Rappelons tout d'abord la description du morphisme (3.2.5).

Lemme 3.3.1 ([2], VI, Prop. 3.1.3). Supposons que $Y^{\prime}$ soit défini par une suite régulière $t_{i}, \ldots, t_{r}^{\prime}$ de sections de $\theta_{X}$. Alors l'image d'une section a de $\Omega_{Y}^{j-r} / w_{n}$ par le morphisme (3.2.5) est la section 


$$
\alpha \wedge d t_{1}^{\prime} / t_{1}^{\prime} \wedge \ldots d t_{r}^{\prime} / t_{r}^{\prime}
$$

de $\underline{H}_{Y}^{r},\left(\Omega_{X}^{j}, W_{n}\right)$.

Rappelons que dans cet énoncé, on a identifié $\underline{H}_{Y^{\prime}}^{r}\left(\Omega_{X^{\prime}}^{j} / W_{n}\right)$ au module des parties polaires de $\Omega_{\mathrm{X}}^{j} / w_{n}$ en les $t_{1}^{\prime} \ldots, t_{r}^{\prime} i . e$. le module

$$
\Omega_{X^{\prime} / w_{n}^{j}}^{j}\left[t_{1}^{\prime-1}, \ldots, t_{r}^{\prime-1}\right] / \sum_{i} \Omega_{X^{\prime} / w_{n}^{j}}^{j}\left[t_{1}^{\prime-1}, \ldots, \hat{t}_{i}^{-1}, \ldots, t_{r}^{\prime-1}\right] .
$$

On va donner une description analogue pour le morphisme (3.2.13).

Calcul de $\underline{H}_{Y}^{r}\left(w_{n} \Omega_{X}^{j}\right)$.

Pour toute suite $i_{0}, \ldots, i_{m}$ d'entiers appartenant à la suite $1, \ldots, r$ on notera $j_{i_{0} \ldots i_{m}}$ le morphisme d'immersion de l'ouvert $u_{i_{0}} \ldots i_{m}$ d'inversibilité de $t_{i_{0}}, \ldots t_{i_{m}}$ dans $x$.

Notons immédiatement que dire que $t_{j}$ est inversible est équivalent à dire que $t_{j}$ est inversible. Il $y$ a donc identité entre $U_{1, \ldots, r}$ et $X-Y$.

on posera

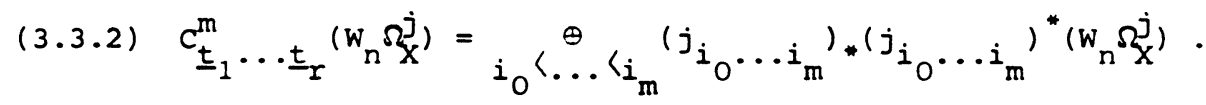

On a de manière naturelle un complexe

$$
\text { (3.3.3) } 0 \longrightarrow w_{n}{ }^{2} x_{x}^{j} \rightarrow c_{t_{1}}^{\cdot}, \ldots . t_{r}\left(w_{n} \Omega_{x}^{j}\right) \rightarrow 0
$$

avec $W_{n} \Omega_{X}^{j}$ placé en degré 0 . Ce complexe est isomorphe dans $D(z$-Mod) à $R_{-Y}^{I}\left(W_{n} \nu_{X}^{j}\right)$.

D'autre part, si l'on note $\operatorname{Kosz}^{\cdot(m)}\left(w_{n} \Omega^{j}\right)$ le complexe de Koszul de $w_{n} s^{j}$ relativement à la suite $t_{1}^{m}, \ldots, t_{r}^{m}$ placé entre les

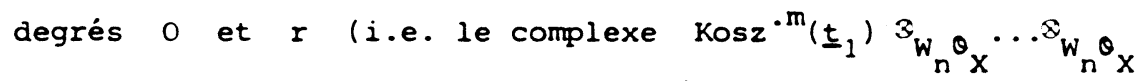
$\operatorname{Kosz}^{\cdot(m)}\left(t_{r}\right) \varepsilon_{W_{n} \theta_{X}} w_{n} \Omega^{j}$ où $\operatorname{kosz}^{\cdot(m)}\left(\underline{t}_{i}\right)$ désigne le complexe de Koszul de l'anneau $w_{n}{ }^{\theta} x$ relativement à l'élément $t_{i}^{m}$ de $\left.w_{n}^{\theta} x\right)$, le complexe $(3.3 .4)$ est isomorphe au complexe $\frac{\lim }{m} \operatorname{Kosz}^{\cdot(m)}\left(w_{n} \Omega_{x}^{j}\right)$ 
où pour $m^{\prime} \leqslant m$ le morphisme de transițion Kosz.( $\left.{ }^{\prime}\right)\left(w_{n} \Omega^{j} x^{j}\right) \longrightarrow$ $\mathrm{Kosz}^{\cdot(m)}\left(w_{n} \Omega_{X}^{j}\right)$ est donné en degré $m$ et sur la composante d'indice $i_{1}, \ldots, i_{r-m}$ par la multiplication par $\left(t_{j_{1}} \cdots t_{j_{m}}\right)^{m^{\prime}-m}$ où $j_{1}, \ldots, j_{m}$ est la suite complémentaire de la suite $i_{1} \ldots, i_{r-m}$. on en déduit en particulier un isomorphisme

$$
\text { (3.3.4) } \quad \mathrm{H}_{Y}^{\Gamma}\left(\mathrm{w}_{n} \Omega_{X}^{j}\right) \stackrel{\lim }{\mathrm{m}} \mathrm{W}_{\mathrm{n}} \Omega_{\mathrm{X}}^{j} / \mathrm{I}{ }^{(\mathrm{m})} \mathrm{W}_{\mathrm{n}} \Omega_{\mathrm{X}}^{j}
$$

où $I^{(m)}$ est l'idéal de $w_{n}^{\theta} x$ engendré par $t_{1}^{m} \ldots, t_{r}^{m}$. Notant $1 / t_{1}^{m} \cdots t_{r}^{m}$ l'image de la section unité de $w_{n}{ }^{\theta} x^{/ I}(m)$ par le morphisme canonique $w_{n}^{\theta} x^{/ I}{ }^{(m)} \longrightarrow \frac{\lim }{m} w_{n} x^{/ I}{ }^{(m)}$, on voit que (3.3.5) s'écrit comme un isomorphisme

(3.3.6) $\quad \underline{H}_{Y}^{Y}\left(w_{n} \Omega_{X}^{j}\right) \stackrel{\sim}{\longrightarrow} w_{n} \Omega_{X}^{j}\left[t_{1}^{-1}, \ldots, t_{x}^{-1}\right] / \sum_{i} w_{n} \Omega_{X}^{j}\left[t_{1}^{-1}, \ldots, \hat{t}_{i}^{-1}, \ldots, t_{I}^{-1}\right]$. On déduit que $\omega \wedge d t_{1} / t_{1} \wedge \ldots \wedge d t_{1} / t_{r}$ est bien une section de $\underline{H}_{Y}^{r}\left(W_{n} \Omega_{X}^{j}\right)$.

Tirons un corollaire de la description (3.3.6).

Corollaire 3.3 .7 . On a un isomorphisme canonique

(3.3.8) $\quad \mathrm{H}_{Y}^{\Gamma}\left(\mathrm{w}_{n} \theta_{X}\right) \otimes_{\mathrm{w}_{n} \theta_{X}} \mathrm{w}_{n} \Omega_{X}^{j} \stackrel{\sim}{\longrightarrow} \mathrm{H}_{Y}^{Y}\left(\mathrm{w}_{n} \Omega_{X}^{j}\right)$.

Proposition 3.3.9. Supposons que $Y$ soit défini par une suite régulière $t_{1}, \ldots, t_{r}$ de sections de $\theta_{X}$. Alors 1 'image d'une section $w$ de $W_{n} \Omega_{Y}^{j-r}$ par le morphisme $(3.2 .13)$ est la section

$(3.3 .10) \quad \omega \wedge d t_{1} / t_{1} \wedge \ldots \wedge d t_{1} / t_{1}$

de $\underline{H}_{Y}^{r}\left(w_{n} \Omega \Omega_{X}^{j}\right)$.

Démonstration : La question est locale. On peut donc supposer qu'il existe un morphisme $g$ étale de $X$ vers $A_{k}^{a}$ tel que $Y$ soit le produit cartésien $\mathbb{A}_{k}^{b} X_{A_{k}^{a}} X$. Notons $h$ la restriction de $g$ à $Y$, $c^{\prime}$ est un morphisme étale, on a donc ${ }^{n}{ }^{*} w_{n} \Omega_{Y} \stackrel{\sim}{\longrightarrow} w_{n} \xi_{k}^{b}$. La section $i$ : s'obtient donc comme combinaison $w_{n}{ }^{\theta} x^{-1 i n e ́ a i r e ~ d e ~ s e c t i o n s ~} u "$ de $\theta_{A_{k}}$. Il suffit donc de prouver la proposition 3.3 .9 dans la situation $X=A_{k}^{a}, Y=A_{k}^{b}$. On a alors $X^{\prime}=\operatorname{spec}\left(W_{n}\left[t_{1}, \ldots, t_{a}^{\prime}\right]\right)$ et $Y^{\prime}$ donné 
par $t_{1}^{\prime}=\ldots=t_{r}^{\prime}=0$. De plus, le morphisme de Frobenius de $x$ est relevé par le morphisme $F$ de $\theta^{\prime}$ défini par $F\left(t_{i}^{\prime}\right)=t_{i}^{\prime}$. On a alors un morphisme canonique

$$
\text { (3.3.11) } \varphi: \Omega_{\mathrm{X}}^{\cdot} / \mathrm{w}_{\mathrm{n}} \longrightarrow \mathrm{w}_{\mathrm{n}} \Omega_{\mathrm{X}}^{\dot{0}}
$$

induit par le morphisme

$$
(3.3 .12) \theta_{X^{\prime}} \rightarrow w_{n} \theta_{x}
$$

envoyant $t_{i}^{\prime}$ sur $t_{i}=\left(t_{i}^{\prime} \bmod p, 0,0, \ldots\right)$

$\varphi$ induit un morphisme

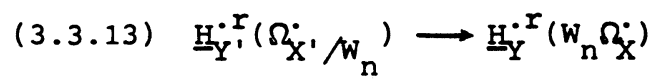

qui admet donc la description suivante : à $\sum_{i} \mu_{i} \cdot\left(1 / t^{\prime}\right)^{i}$, on associe $\sum_{i} \varphi\left(\mu_{i}\right) \cdot(1 / \underline{t})^{i}$ où 1 'on a abrégé $1 / t_{1}^{\prime i} 1 \ldots 1 / t_{r}^{\prime i} r$ en $\left(1 / t^{\prime}\right)^{i}$, de même avec les $t$.

Appliquant $\underline{H}^{*}(-)$ au morphisme $(3.3 .13)$, on obtient un morphisme

$$
(3.3 .14) \quad \underline{H}^{*}\left(\underline{H}_{Y}^{\cdot} \cdot r\left(\Omega_{X}^{*} / W_{n}\right)\right) \rightarrow \underline{H}^{*}\left(\underline{H}_{Y}^{\cdot r}\left(W_{n} \Omega_{X}^{*}\right)\right)
$$

qui n'est autre que le morphisme obtenu en appliquant $\underline{H}_{Y}^{.}(-)$au morphisme

$$
(3.3 .15) \quad \underline{H}^{*}((3.3 .11)): \underline{H}^{*}\left(\Omega_{X}^{*} / w_{n}\right) \longrightarrow \underline{H}^{*}\left(w_{n} \Omega_{X}^{*}\right) \text {. }
$$

Ce dernier morphisme est un isomorphisme, en effet, c'est le composé de l'isomorphisme (3.2.12) et de l'isomorphisme de Cartier

$$
\text { (3.3.16) } w_{n} \Omega_{X}^{*} \stackrel{\sim}{\longrightarrow} \underline{H}^{*}\left(w_{n} \Omega_{X}^{*}\right) \text {. }
$$

On peut maintenant, suivant les constructions pas à pas, donner la description de $(3.2 .13)$ : soit $\omega \in w_{n} \Omega^{j-r} \sim \underline{H}^{j-r}\left(\Omega_{Y}^{j} / w_{n}\right)$, on représente $\omega$ par un cycle $\alpha$ de $\Omega_{Y}^{j-r} / w_{n}$. L'image de $\alpha$ par $(3.2 .5)$ est a $d t_{1}^{\prime} / t_{1}^{\prime} \ldots d t_{r}^{\prime} / t_{r}^{\prime}$, c'est un cycle, qui définit donc une classe de cohomologie dans $\underline{H}^{*}\left(\underline{H}_{Y}^{*} \cdot\left(\Omega_{X}^{*} / w_{n}\right)\right)$ et cette classe n'est autre (d'après la description de (3.3.13)) que la 
M. GROS

classe de $\varphi(\alpha) \wedge d t_{1} / t_{1} \wedge \ldots \wedge d t_{1} / t_{1}$ soit $\omega \wedge d t_{1} / t_{1} \wedge \ldots \wedge d t_{1} / t_{1}$. D'où la proposition 3.3 .9 .

Comme $d\left(d t_{1} / t_{1} \wedge \ldots \wedge d t_{1} / t_{1}\right)=0$, on en déduit que $(3.2 .4)$ fournit bien un morphisme $(3.2 .3)$.

Corollaire $3 \cdot 3 \cdot 17$. Les deux diagrames suivants sont commutatifs

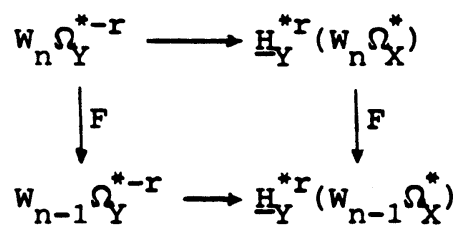

$(3.3 .19)$

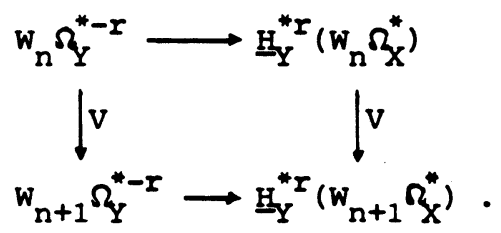

Démonstration : Il suffit d'utiliser la proposition 3.3 .3 en remarquant que $F\left(d t_{1} / t_{1} \wedge \ldots \wedge d t_{1} / t_{I}\right)=d t_{1} / t_{1} \wedge \ldots \wedge d t_{I} / t_{I}$.

Remarque. On peut se demander ce qu'il advient lorsque l'on applique le foncteur $\underline{H}^{*}(-)$ au morphisme $(3.2 .3)$ et que 1 'on utilise 1 'opération de Cartier (3.3.16). D'après $([21,1.3 .9])$, la filtration canonique (loc. cit.) de $w_{n} \Omega_{\dot{x}}^{\circ}$ a un gradué extension de $\theta_{x}$-Modules localement libres. Par suite, il en est de même (grâce à l'isomorphisme $(3 \cdot 3 \cdot 16))$ de $\left.\underline{H}^{*}\left(w_{n} \Omega_{X}^{*}\right)\right)$. D'où

$(3.3 .20) \quad \frac{i}{H_{Y}}\left(w_{n} \Omega_{X}^{*}\right)=\underline{H}_{Y}^{i}\left(\underline{H}^{*}\left(w_{n} \Omega_{X}^{*}\right)\right)=0$ pour $i \neq x$.

comme $z W_{n} \Omega_{X}^{*}$ et ${ }^{B W} \Omega_{x}^{*}$ sont des faisceaux quasi-cohérents, on en déduit que

(3.3.21) $\mathrm{H}_{Y}^{i}\left(\mathrm{ZW}_{n} \Omega_{X}^{*}\right)=\mathrm{H}_{Y}^{i}\left(\mathrm{BW}_{n} \Omega_{X}^{*}\right)=0$ pour $i$ suffisamment grand.

On dispose donc grâce à 3.1 .19 d'un isomorphisme canonique

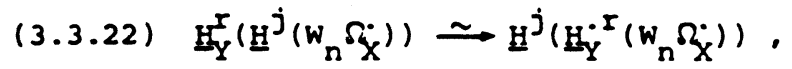

soit encore, utilisant $(3.3 .16)$ un isomorphisme

(3.3.23) $\underline{H}_{Y}^{\mathbf{Y}}\left(w_{n} \Omega_{X}^{j}\right) \stackrel{\sim}{\longrightarrow} H^{j}\left(\underline{H}_{Y}^{\cdot \Gamma}\left(w_{n} \Omega_{X}^{*}\right)\right)$. 
Utilisant la description donnée par la proposition 3.3.3, on en déduit que $l$ 'on a un diagramme commutatif

$$
\begin{aligned}
& w_{n} \Omega_{Y}^{*-r} \longrightarrow(3.2 .4) \longrightarrow H_{Y}^{r}\left(w_{n} \Omega_{X}^{*}\right) \\
& (3.3 .16) \\
& 2(3.3 .23) \\
& \underline{H}^{*}-r\left(w_{n} \Omega_{Y}^{*} \quad \overrightarrow{H^{*}((3.2 .4))} \underline{H}^{*}\left(\underline{H}_{Y}^{* r}\left(w_{n} \Omega_{X}^{*}\right)\right)\right.
\end{aligned}
$$

résultant du fait que $F\left(d t_{1} / t_{1} \ldots d t_{r} / t_{r}\right)=d t_{1} / t_{1} \ldots d t_{r} / t_{r}$.

\subsection{Comparaison avec la définition 1.2.6.}

Lorsque $f$ est une immersion fermée, le morphisme $f_{*}$ de 1.2.6 fournit par adjonction un morphisme

$$
W_{n} \Omega_{Y} \rightarrow R I_{Y}\left(W_{n} \Omega_{X}^{*}\right)(r)[r] \text {. }
$$

Compte tenu de $(3 \cdot 3.20)$, on a un isomorphisme

$$
R \Gamma_{Y}\left(W_{n} \Omega_{X}^{*}\right)(r)[r] \stackrel{\sim}{\sim} H_{Y}^{\cdot r}\left(W_{n} \Omega_{X}^{*}(r)\right) .
$$

(3.4.1) s'interprète donc comme un morphisme

$$
\text { (3.4.3) } \quad \mathrm{w}_{n} \Omega_{Y}^{\circ} \longrightarrow \mathrm{H}_{Y}^{.}\left(\mathrm{w}_{n} \Omega_{X}^{*}\right)(r)
$$

dans la catégorie des $w_{n} \Omega_{x}$-Modules différentiels gradués. On va le comparer avec le morphisme $(3.2 .3)$. Il suffit, vu la $w_{n} \Omega_{x}$-linéarité de comparer $I^{\prime}$ image de $1 \in H^{O}\left(Y, W_{n} \theta_{Y}\right)$ par $H^{O}(X,(3.4 .3))$ et par $H^{O}(X,(3.2 .3))$ dans $H_{Y}^{r}\left(X, w_{n} \Omega_{X}^{r}\right)$.

On va pour ce faire passer par l'intermédiaire de la cohomologie cristalline. Il résulte de la comparaison entre le théorème de dualité d'Ekedahl et le théorème de dualité cristallin (cf. [10]) que le diagramme suivant est commutatif

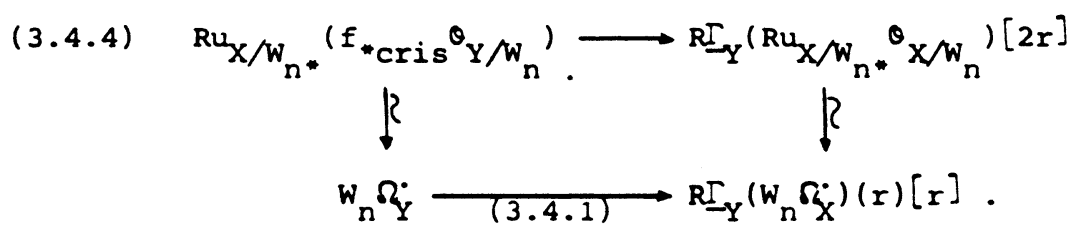

Les morphismes verticaux sont induits par le théorème de comparaison (Thm. 1.4, II de [21]) et le morphisme horizontal supérieur est la 
M. GROS

projection par $R u_{X / W_{n *}}$ du morphisme de Gysin (3.3.8) du chapitre VI de $[1]$.

D'autre part, d'après le théorème 3.3.5 du chapitre vi de [2], le diagramme suivant est commutatif

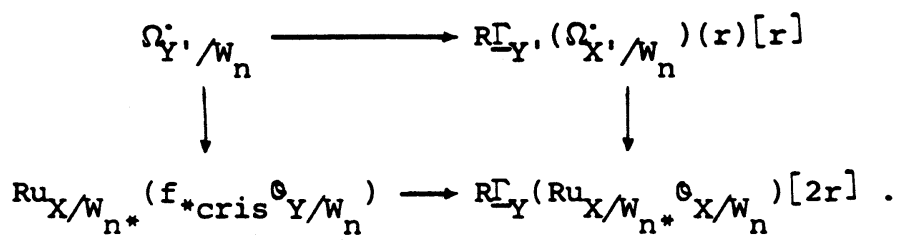

Le morphisme horizontal inférieur étant celui figurant dans (3.4.4), le morphisme horizontal supérieur est le morphisme (3.2.5) réinterprété grâce à l'isomorphisme canonique

$$
R_{-Y} \cdot\left(\Omega_{X}^{*} / W_{n}\right)(r)[r] \rightarrow \underline{H}_{Y}^{\cdot} \cdot r\left(\Omega_{X}^{*} / W_{n}\right)(r)
$$

résultant de ce que $\underline{H}_{Y}^{\cdot} \cdot{ }^{j}\left(\Omega_{X}^{*} / W_{n}\right)=0$ sauf pour $j=r$.

Les morphismes verticaux sont les morphismes canoniques.

Mettant (3.4.4) et (3.4.5) ensemble, puis prenant la cohomologie locale on obtient un diagramme commutatif

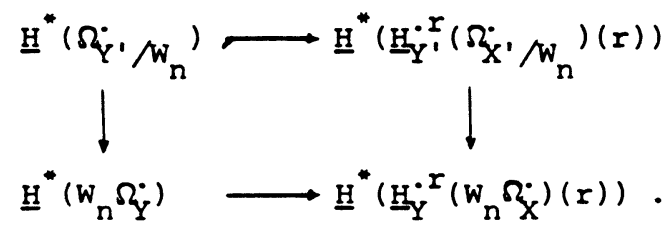

Diagramme que l'on réécrit (par définition de (3.2.3))

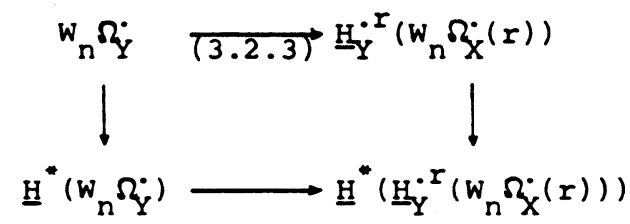

Ce qui joint au diagrame commutatif (3.3.24) montre que $H^{*}\left(X_{1}(3.4 .3)\right)$ et $H^{* \prime}(X,(3.2 .3))$ coincide. D'où le résultat (en faisant * =0). 


\subsection{Cas de la cohomologie logarithmique.}

Le morphisme $(3.2 .4)$ induit un morphisme de pro-Modules gradués compatibles (d'après le corollaire 3.3 .17 ) à $F$ et $V$

$$
\text { W. } \Omega_{Y}^{*} \longrightarrow \mathrm{H}_{Y}^{*} \mathrm{r}\left(\mathrm{W} . \Omega_{\mathrm{X}}^{*}\right)(\mathrm{r})
$$

qui sera encore noté $f_{*}$. On a un diagramme commutätif de pro-objets

$$
\begin{aligned}
& \text { W. } \Omega_{Y}^{*} \longrightarrow \mathrm{H}_{Y}^{*}\left(\mathrm{~W} \cdot \Omega_{X}^{*}\right)(\mathrm{r}) \\
& \downarrow 1-\mathrm{F} \quad \mid 1-\mathrm{F} \\
& \mathrm{W} \cdot \Omega_{Y}^{*} \longrightarrow \mathrm{H}_{Y}^{*}\left(\mathrm{~W} \cdot \Omega_{X}^{*}\right)(\mathrm{r}) .
\end{aligned}
$$

D'autre part, la suite exacte (1.3.2) de I induit une suite exacte

(3.5.3) $0 \longrightarrow \mathrm{H}_{Y}^{Y}\left(\mathrm{~W} . \Omega_{X, 10 g}^{*}\right) \rightarrow \mathrm{H}_{Y}^{Y}\left(\mathrm{~W} . \Omega_{X}^{*}\right) \underset{1-\mathrm{F}}{\longrightarrow} \mathrm{H}_{Y}^{Y}\left(\mathrm{~W} . \Omega_{X}^{*}\right) \longrightarrow$

$$
\mathrm{H}_{\mathrm{Y}}^{\mathrm{r}+1}\left(\mathrm{~W} . \Omega_{\mathrm{X}, \log }^{*}\right) \rightarrow 0
$$

et $\underline{H}_{Y}^{i}\left(w \cdot \Omega_{X, 10 g}^{*}\right)=0$ pour tout $i \neq r, r+1$.

Remarque. Il est en général faux que $\underline{H}_{Y}^{r+1}\left(w . \Omega_{X}^{*} \log \right)=0$ sauf si $Y$ est réduit à un point fermé. Le lecteur trouvera des compléments intéressants sur ces questions dans [15].

Prenant le morphisme induit sur le noyau des flèches verticales de (3.5.2), on obtient un morphisme

(3.5.4) $f_{*}: W . \Omega_{Y, \log }^{*} \rightarrow \mathrm{H}_{Y}^{I}\left(W \cdot \Omega_{X, \log }^{*}(r)\right)$.

Tensorisant ce morphisme par $z / p^{n}$, on obtient (compte tenu de 1 'isomorphisme $(1.3 .6)$ de I) un morphisme

$$
\text { (3.5.5) } f_{*}: W_{n} R_{Y, \log }^{*} \rightarrow H_{Y}^{Y}\left(w_{n} R_{X, \log }^{*}(r)\right) \text {. }
$$

Proposition 3.5.6. Supposons que $Y$ soit défini dans $X$ par une suite régulière $t_{1} \ldots, t_{r}$ de sections de $\theta_{X}$. Alors l'image d'une section a de $W_{n} \Omega_{Y, l o g}^{j-r}$ par le morphisme (3.5.5) est la section

$$
\alpha \wedge d \underline{t}_{1} / t_{1} \wedge \ldots \wedge d t_{1} / t_{1}
$$

de $\frac{H_{Y}^{r}}{Y}\left(w_{n} \Omega_{X, 10 g}^{j}\right)$. 
Démonstration : La proposition résulte immédiatement de la construction de (3.5.5) et de 3.3.9.

(3.5.5) est donc un morphisme de $w_{n} \Omega_{X, l o g}^{*}$-Modules gradués. Théorème $3.5 .8(3.5 .5)$ est un isomorphisme.

Démonstration : Commençons par prouver le théorème lorsque $n=1$. La question étant locale, on peut trouver des sections $t_{1}, \ldots, t_{N}$ de $\theta_{X}$ telles que $d t_{1} \ldots . d t_{N}$ forment une base de $\Omega_{X}^{1}$ et que $Y$ soit donné par les équations $t_{1}=\ldots=t_{r}=0$. Prouvons tout d'abord l'injectivité de (3.5.5). Par construction, il suffit de prouver l'injectivité de (3.2.13) (lorsque $n=1$ ). Soit donc $\omega \in \Omega^{j-r}$, son image par $(3.2 .13)$ est la classe de la section $\omega \wedge d t_{1} / t_{1} \ldots d t_{r} / t_{r}$ dans $\underline{H}_{Y}^{r}\left(\Omega_{X}^{j}\right)=\Omega_{X}^{j}\left[t_{1}^{-1}, \ldots, t_{I}^{-1}\right] \Sigma \delta_{X}^{j}\left[t_{1}^{-1}, \ldots, \hat{t}_{i}^{-1}, \ldots, t_{I}^{-1}\right]$. Dire que cette classe est nulle, c'est donc dire que l'on a une égalité

$$
\omega \wedge d t_{1} / t_{1} \ldots d t_{r} / t_{r}=\sum_{j} \alpha_{j} / t_{1} \ldots \hat{t}_{j} \ldots t_{r}
$$

dans $\Omega_{X}^{j}\left[t_{1}^{-1}, \ldots, t_{r}^{-1}\right]$. On écrit alors

$(3.5 .10) \quad \omega=\sum_{\left(i_{1}, \ldots . i_{j-r}\right)} \omega_{i_{1} \ldots i_{j-r}} d t_{i_{1}} \ldots d t_{i_{j-r}}$

(la sommation étant prise sur tous les multi-indices $\left(i_{1} \ldots \ldots i_{j-r}\right)$ tels que $r\left\langle i_{1} \ldots<i_{j-r}\right.$ et $\left.u_{i_{1}} \ldots i_{j-r} \in \theta_{Y}\right)$.

L'égalité (3.5.9) implique alors une égalité du type (3.5.11) $\omega_{i_{1} \ldots i_{j-r}}=\sum_{\chi=1}^{r} t_{\chi} \alpha_{i}$ pour tout $\left(i_{1}, \ldots . i_{j-r}\right)$ impossible si $\omega_{i_{1} \ldots i_{j-r}} \neq 0$ puisque $Y$ est donné par $t_{1}=\ldots=t_{r}=0$. Passons maintenant à la surjectivité. On pose (3.5.12) $F i l^{i_{H}} r\left(\Omega_{X}^{j}\right)=\left\{x \in \underline{H}_{Y}^{r}\left(\Omega_{X}^{j}\right)\right.$ tel que $x$ admette un représentant $x^{\prime}$ dans $\Omega_{X}^{j}\left[t_{1}^{-1} \ldots . t_{r}^{-1}\right]$ de la forme $x^{\prime \prime} /\left(t_{1} \ldots t_{r}\right)^{p i}$ avec $\left.x^{\prime \prime} \in \Omega_{X}^{j}\right\}$.

On définit ainsi une filtration croissante dont les termes sont nuls pour $i$ négatif ou nul. On notera $F_{i l} j_{\mathrm{H}_{Y}^{r}}^{r}\left(z \Omega_{X}^{j}\right)$ la filtration 
induite (via 1 'inclusion canonique $\left.\underline{H}_{Y}^{Y}\left(z_{X}^{j}\right) \longrightarrow H_{Y}^{j}\left(\Omega_{X}^{j}\right)\right)$. On remarquera d'ailleurs que 3.1 .12 fournit (grầce à (3.3.6)) un isomorphisme $\underline{H}_{Y}^{r}\left(z \Omega_{X}^{j}\right)=z \Omega_{X}^{j}\left[t_{1}^{-p} \ldots ., t_{r}^{-p}\right] \sum z \Omega_{X}^{j}\left[t_{1}^{-p} \ldots \ldots \hat{t}_{i}^{-p}, \ldots, t_{r}^{-p}\right]$ et que

$$
c\left(x^{\prime \prime} /\left(t_{1} \ldots t_{r}\right)^{p i}\right)=c\left(x^{\prime \prime}\right) /\left(t_{1} \ldots t_{r}\right)^{i} \quad \text { pour } \quad x^{\prime \prime} \in z \Omega_{x}^{j}
$$

C est donc de manière évidente un morphisme filtré qui passe au gradué gr $\mathrm{H}_{Y}^{r}\left(\mathrm{z} \Omega_{X}^{j}\right) \longrightarrow \mathrm{gr} \mathrm{H}_{Y}^{r}\left(\Omega_{X}^{j}\right)$. (3.5.13) montre alors que $g r(c)=0$ sauf si $i=1$, ce qui implique que 1 'image de $\xi_{Y}^{r}\left(\Omega_{X}^{j}, \log \right)$ dans $\underline{H}_{Y}^{r}\left(\mathrm{z} \Omega_{\mathrm{X}}^{j}\right)$ est constitué d'éléments admettant un représentant dans $z \Omega_{x}^{j}\left[t_{1}^{-1}, \ldots, t_{r}^{-1}\right]$ de la forme $x^{\prime \prime} /\left(t_{1} \ldots t_{r}\right)^{p}$. L'invariance par $C$ d'un tel élément conduit donc à une égalité de la forme

$$
\left(C\left(x^{\prime \prime}\right)\left(t_{1} \ldots t_{r}\right)^{p-1}-x^{\prime \prime}\right) /\left(t_{1} \ldots t_{r}\right)^{p}=\sum_{i} \theta_{i} /\left(t_{1} \ldots \hat{t}_{i} \ldots t_{r}\right)^{p} \text {. }
$$

soit encore

$$
c\left(x^{\prime \prime}\right)\left(t_{1} \ldots t_{r}\right)^{p-1}-x^{\prime \prime}=\sum_{i=1}^{r} \beta_{i} t_{i}^{p} .
$$

Nous noterons I 1 'ensemble des $j$-uples $\left(i_{1}, \ldots, i_{j}\right)$ d'entiers tels que $i_{\chi} \in[1, N]$ pour tout $\chi \in[1, j]$ et $i_{1}<\ldots<i_{j}$. $D^{\prime}$ autre part, on utilisera $l^{\text {'abréviation suivante }: d t_{i}}=d t_{i_{1}} \ldots d t_{i_{j}}$ pour $\underline{i} \in I$ et $\underline{i}=\left(i_{1}, \ldots . i_{j}\right) ; d \operatorname{dog} t_{\underline{i}}=\operatorname{dlog}_{i_{1}} \ldots \operatorname{llog}_{i_{j}}$ pour ce même $\underline{\underline{i}}$. L'égalité (3.5.15) implique une égalité du type

$$
x^{\prime \prime}=t_{1}^{p-1} x^{\prime \prime \prime}+t_{2}^{p_{\beta}}+\ldots+t_{Y}^{p_{\beta}} .
$$

Comme $d x^{\prime \prime}=0$, on en déduit que

$$
t_{1}^{p-2} d t_{1} \cdot x^{\prime \prime \prime}=t_{1}^{p-1} d x^{\prime \prime \prime}+\sum_{j \geqslant 2} t_{j}^{p} \cdot d \beta j \cdot
$$

Si l'on écrit $x^{\prime \prime \prime}=\sum_{\underline{i} \in I} a_{i} d t_{\underline{i}}$ avec $a_{\underline{i}} \in \theta_{X}$ pour tout $\underline{\underline{i}} \in I$, l'égalité (3.5.16) montre que $\sum_{\underline{i} \in I \mid i_{1} \neq 1} a_{\underline{i}} d t t_{\underline{i}}$ est de la forme $t_{1} \delta i+\sum_{j \geqslant 2} t_{j}^{P_{\delta}} j$

Ecrivant $x^{\prime \prime}=\sum_{\underline{\underline{i}} \in I} b_{\underline{i}} d t$ avec $b_{\underline{i}} \in \theta_{X}$ pour tout $\underline{\underline{i}} \in I$, on en déduit que $\sum_{i \in I \mid i_{1} \neq 1} b_{i} d t_{i}$ est de la forme $t_{1}^{p} \cdot \gamma_{1}+\ldots+t_{I}^{p} \cdot \gamma_{I}$. 
M. GROS

Comme on peut échanger le rôle joué par $t_{1}$ par $t_{2}, \ldots, t_{r}$. on voit que ${ }_{i} \in I-\left\{\sum_{1, \ldots, r\}} b_{i}^{d t_{i}}\right.$ est de la forme $t_{1}^{p_{1} \varepsilon_{1}+\ldots+t_{r}^{p} \cdot \varepsilon_{r}} \cdot$ L'image d'un élément $x$ de $\mathbb{F}_{Y}^{r}\left(\Omega_{x, l o g}^{j}\right)$ par la suite d'inclusions

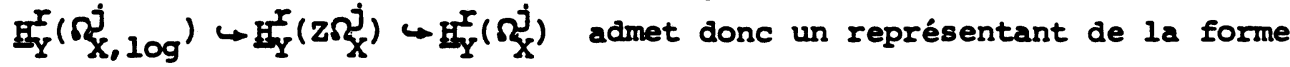
$x^{\prime \prime \prime} d \log t_{1} \wedge \ldots \wedge d \log t_{r}+\left(t_{1}^{p_{1}} x_{1}^{\prime \prime \prime}+\ldots+t_{r}^{p_{x}} x_{r}^{\prime \prime \prime}\right) /\left(t_{1} \ldots t_{r}\right)^{p}$, soit encore un représentant de la forme $z \cdot d t_{1} / t_{1} \ldots d t_{r} / t_{r} \cdot$ On écrit alors $z=\sum_{i} c_{i} d t_{i_{1}} \ldots d t_{i_{j-r}}$ avec $\underline{i}=\left(i_{1} \ldots . i_{j-r}\right)$ tel que $i_{x} \neq 1, \ldots, r$ pour tout $\chi \in[1, j-r]$. Comme par définition on identifie deux représentants $z \cdot d t_{1} / t_{1} \ldots d t_{r} / t_{r}$ et $z^{\prime} \cdot d t_{1} / t_{1} \ldots d t_{r} / t_{r}$ lorsque $z-z^{\prime}=\sum_{i=r}^{r} t_{i} \cdot \mu_{i}$, on en déduit que $l^{\prime}$ ensemble des classes d'éléments de $1 a$ forme $z . d t_{1} / t_{1} \ldots d t_{r} / t_{r}$ est $\Omega^{j-r} \cdot d t_{1} / t_{1} \ldots d t_{r} / t_{r}$ (puisque 1 'on peut supposer que $Y \longleftrightarrow X$ se scinde et que donc $z=z^{\prime}+\sum_{i=1}^{r} t_{i} z_{i}+\sum_{i=1}^{r} z_{i}^{\prime} d t_{i}$ avec $\left.z^{\prime} \in \Omega_{Y}^{j-r}\right)$. L'invariance par $c^{-1}$ implique alors que $z \in \Omega_{Y, l o g}^{j-r}$. D'où le théorème lorsque $n=1$. Pour prouver le théorème pour tout $n$, on va utiliser la filtration p-adique de $w_{n} R_{Y, l o g}^{*}$ dont le gradué est isomorphe a $R_{Y, l o g}^{*}$. Il faut vérifier que le foncteur $H_{Y}^{r}(-)$ est exact sur la suite exacte

$(3.5 .17) 0 \longrightarrow$ w. $\Omega_{x, \log }^{*} \vec{p}$ w. $\Omega_{x, \log }^{*} \rightarrow \Omega_{x, \log }^{*} \rightarrow 0$

i.e. vérifier que $H_{Y}^{r}\left(w . \Omega_{X, l o g}^{*}\right) \rightarrow H_{Y}^{r}\left(\Omega_{X, l o g}^{*}\right)$ est surjectif. Ce qui résulte trivialement de la surjectivité de $(3.5 .5)$ pour $n=1$.

On prendra garde au fait que la non-nullité de $H_{Y}^{r+1}\left(w_{n} \Omega_{x, l o g}^{*}\right)$ montre que $I^{\prime}$ on $n^{\prime}$ a pas d'isomorphisme entre $\mathbb{H}_{Y}^{r}\left(w_{n} \Omega_{X}^{*}, g^{\prime}\right)^{n}$ et $R \Gamma_{Y}\left(W_{n} \Omega_{X, l o g}^{*}\right)[-r]$. Par suite, lorsque $f$ est une immersion fermée, l'application induite par la définition (1.2.9)

$(3.5 .18) \quad f_{*}: w_{n} \Omega_{Y, \log }^{*} \rightarrow R \Gamma_{Y}\left(w_{n} \Omega_{x, \log }^{*}(r)\right)[r]$

n'est pas un isomorphisme.

Néanmoins, la nullité de $H_{Y}^{i}\left(w_{n} R_{X}^{*} \log ^{\prime}\right)$ pour $i$ r montre que $_{r}$ l'on a un isomorphisme

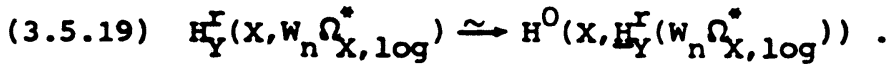




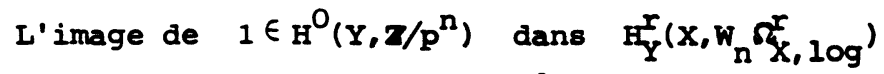
(ou dans $\mathrm{H}^{\mathrm{Y}}\left(\mathrm{X}, \mathrm{w}_{\mathrm{n}} \delta_{\mathrm{X}}^{\mathrm{T}}, \log ^{2}\right)$ par $\mathrm{H}^{0}(\mathrm{X},(3.5 .5))$ sera notée $\mathrm{cl}(\mathrm{Y} / \mathrm{X})$ et appelée classe de cohomologie de $Y$.

L'image de cette classe par le morphisme canonique

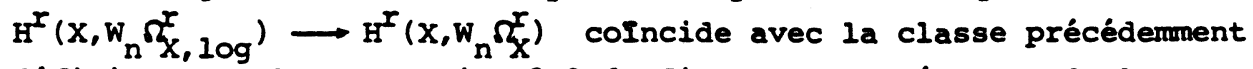
définie avant le corollaire 2.2.6. C'est une conséquence de la construction de $(3 \cdot 5 \cdot 5)$.

Prouvons maintenant 2.3.2. On remarque qu'il suffit de prouver (par factorisation de $f_{*}$ et $f_{*}^{\prime}$ ) la commutativité du diagrame

$$
\begin{aligned}
& W_{n} \Omega_{Y, I 0 g}^{*} \quad f_{*} \quad H_{Y}^{r}\left(W_{n} \Omega_{X, l o g}^{*}\right) \\
& \mathrm{lg}^{*} \quad \mathrm{ln}^{*} \quad \mathrm{r}=\operatorname{codim}(\mathrm{Y})
\end{aligned}
$$

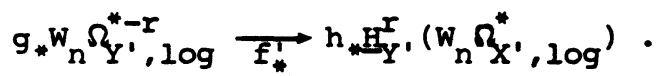

On utilise pour ce faire la description donnée par la propositioii 3.5 .6 en remarquant que si $t_{1} \ldots ., t_{r}$ est une suite régulière de sections de $\theta_{X}$ définissant localement $Y$ dans $X$, alors $h^{*} t_{1} \ldots, h^{*} t_{r}$ est une suite régulière de sections de $\theta_{X}$ définissant localement $Y^{\prime}$ dans $X^{\prime}$. La commutativité est alors claire.

Prouvons maintenant 2.5.1. On va effectuer un calcul à la Čech des deux membres de $(2.5 .2)$. Soit donc $u=\left(U_{j}\right)_{j \in I}$ un recouvrement de $X$ par des ouverts affines $U_{j}$ tels que $U_{j} \cap_{Y}$ soit défini par une section $t_{(j)}$ de $\theta_{x}$.

Par définition, $t_{(j)} / t_{(i)}$ est un cocycle représentant la classe de $J$ dans $P i c(X) \cdot c_{1}(J)$ est donc représenté par la classe du 1 -cocycle $\operatorname{dlog}\left(t_{(i)} / t_{(j)}\right)(i<j)$ dans $\mathrm{H}^{1}\left(x, w_{n} \Omega^{1}, \log \right) \stackrel{\sim}{\longrightarrow}$ $\mathrm{H}^{1}\left(u, w_{n} \Omega_{X}^{1}, \log ^{\prime}\right)$. D'autre part, $f_{*}(1)$ est représenté par l'image par la différentielle $\delta$ du complexe de čech de $w_{n} \Omega^{1}, l o g$ du o-cocycle (d'après 3.5.6) $\operatorname{dlog}\left(t_{(i)}\right)$, soit $\operatorname{dlog}\left(t_{(i)}\right)-\operatorname{dlog}\left(t_{(j)}\right)=$ $-\operatorname{dlog}\left(t_{(j)} / t_{(i)}\right)$ avec $i>_{j} \cdot D^{\prime o u ̀ ~}(2.5 .2)$. 
M. GROS

4. Classe de cohomologie d'un cycle sinqulier.

\subsection{Définition.}

Proposition 4.1.1. Soit $Y$ un sous-schéma fermé intègre de $X$. On a (4.1.2) $\quad H_{Y}^{j}\left(x, w_{n} \Omega \Omega_{x, l o g}^{j}\right)=H_{Y}^{j+1}\left(x, w_{n} \Omega_{x, l o g}^{j}\right)=0$

pour $j<\operatorname{codim}(Y)=r$.

Démonstration : i) $Y$ est lisse. On a une suite spectrale

$$
E_{2}^{a b}=H^{a}\left(X, H_{Y}^{b}\left(w_{n} \Omega_{X}^{j}, \log \right)\right) \Longrightarrow H_{Y}^{*}\left(X, w_{n} \Omega_{X, l o g}^{j}\right)
$$

et d'après $(3.5 .3) \quad E_{2}^{a b}=0$ pour tout $b \neq r, r+1$.

Le theorème (3.5.8) montre que $E_{2}^{a r}=H^{a}\left(x, W_{n} \Omega_{Y, l o g}^{j-r}\right)$.

D'où $E_{2}^{a r}=0$ pour $j<r$. On vérifie donc qu'il y a suffisamment de termes nuls dans la suite spectrale (4.1.3) pour affirmer (4.1.2).

ii) Cas qénéral. On va prouver (4.1.2) par récurrence descendante sur la codimension de $Y$.

Supposons $Y$ de codimension $r$ et la proposition prouvée pour les codimensions supérieures ou égales à $r+1$. Soit $Y^{\prime}$ le lieu singulier, de sorte que $Y-Y^{\prime}$ est lisse de codimension $r$. On utilise alors la suite exacte longue de cohomologie

$$
\begin{aligned}
& \ldots \ldots . . \rightarrow H_{Y}^{r}\left(x, w_{n} \Omega_{X, l o g}^{j}\right) \rightarrow H_{Y}^{r}\left(x, w_{n} \Omega_{X, l o g}^{j}\right) \rightarrow H_{Y-Y}^{r}\left(x, w_{n} \Omega_{X, l o g}^{j}\right) \rightarrow \\
& H_{Y}^{r+1}\left(x, w_{n} \Omega_{X, l o g}^{j}\right) \rightarrow \ldots
\end{aligned}
$$

(où l'on a utilisé l'isomorphisme d'excision évident

$$
\text { (4.1.5) } \left.\mathrm{H}_{Y-Y^{\prime}}^{*}\left(X-Y^{\prime}, W_{n} \Omega_{X, l o g}^{*}\right)=H_{Y-Y^{\prime}}^{*}\left(X, W_{n} \Omega_{X, 1 \circ g}^{*}\right)\right)
$$

qui, jointe à l'hypothèse de récurrence et au fait que (4.1.2) est prouvé pour $Y-Y$ ' fournit le résultat.

Pour $j=r,(4.1 .4)$ montre en particulier que l'on a un isomorphisme

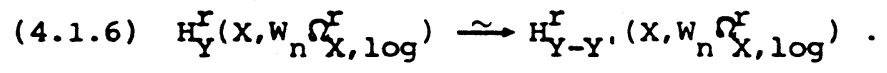

Définition 4.1.7. On appelle classe de cohomologie de $Y$ dans $X$. l'image par (4.1.6) de la classe de $Y-Y^{\prime}$ dans $X$. 
Lorsque $Y$ est lisse, elle coIncide bien entendu avec la classe de cohomologie précédemment définie.

Utilisant les morphismes canoniques $\mathrm{H}_{Y}^{r}\left(X, W_{n} \Omega^{x}, \log \right) \longrightarrow$ $H^{r}\left(x, w_{n} \Omega_{x, l o g}^{r}\right)$ et $H^{r}\left(x, w_{n} \Omega_{x, l o g}^{r}\right) \rightarrow H^{2 r}\left(x, w_{n} \Omega_{x}^{*}\right)=H_{c r i s}^{2 r}\left(x / w_{n}\right)$, on dispose ainsi d'une classe de cohomologie cristalline d'un sousschéma fermé intègre éventuellement singulier. Lorsque $\mathrm{Y}$ est lisse, la classe construite ici coincide avec celle de Berthelot $([2], V I, 3.1)$ corme il résulte du diagranme (3.4.4) du chapitre II et du principe de construction $\left(c l(Y / X)=i_{*}\left(1_{Y}\right)\right)$. Bien entendu, si $Y=\sum n_{i} Y_{i}$ avec $n_{i} \in \mathbb{Z}$ et $Y_{i}$ des sous-schémas fermés intègres, $c l(Y / X)$ désignera $\sum n_{i} c l\left(Y_{i} / X\right) \in \mathrm{H}^{r}\left(X, W_{n} \Omega^{r} X, l o g\right)$ (ou $\left.H_{\text {Cris }}^{2 r}\left(X / W_{n}\right)\right), r$ désignant la codimension commune des $Y_{i}$.

Pour $n=1$, on obtient par œ procédé une classe de cohomologie d'un cycle éventuellement singulier en cohomologie de Hodge et de De Rham d'un schéma défini sur un corps parfait complétant ainsi les constructions d'El zein (cf. [11]).

\subsection{Propriétés.}

Proposition 4.2.1. Si $Y$ est un diviseur de Cartier de $X$, on a (4.2.2) $\quad c l(Y / X)=c_{1}\left(\theta_{X}(Y)\right)$ dans $H^{1}\left(X, W_{n} \Omega_{X, 10 g}^{1}\right)$.

Démonstration : Par définition de chacun des deux membres, on est ramené au cas où $Y$ est lisse. La proposition résulte alors de $(2.5 .2)$.

Proposition 4.2.3. Soient $f: X \longrightarrow Y$ un morphisme propre entre k-schémas lisses et $z$ un sous-schéma fermé de $X$ de codimension $r$. on a alors

$$
\text { (4.2.4) } \quad c l\left(f_{*} Z / Y\right)=f_{*} c l(Z / X) \in H_{f(Z)}^{r+N}\left(Y, W_{n} \Omega_{Y, l o g}^{r+N}\right)
$$

où $\quad N=\operatorname{dim}(Y)-\operatorname{dim}(X)$.

Démonstration : Posons $T=f(z)$. Grâce à $(4.1 .2)$ et $(4.1 .4)$, on voit que $l^{\prime}$ on a des isomorphismes

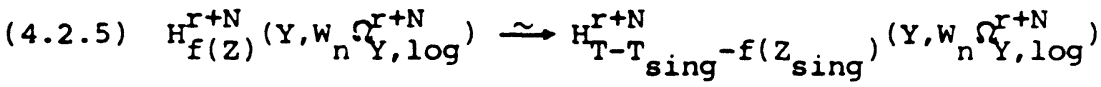

$$
\begin{aligned}
& \underset{51}{\sim} \mathrm{H}_{T^{\prime}}^{r+N}\left(Y^{\prime}, W_{n} \Omega_{Y^{\prime}}^{r+N}, \log \right)^{\prime}
\end{aligned}
$$


I. GROS

avec $T^{\prime}=T-T_{\text {sing }}{ }^{-f\left(Z_{\text {sing }}\right)}$ et $Y^{\prime}=Y-T_{\text {sing }}{ }^{-f\left(Z_{\text {sing }}\right)}$ (l'indice sing indiquant que l'on prend la partie singulière des schémas considérés). Vu la définition des classes de cycles, on est ramené à prouver que

(4.2.6) $\left(f_{*} \operatorname{cl}(Z / X) \Rightarrow f_{*} \operatorname{cl}\left(Z-Z_{\text {sing }} / X\right)=c l\left(T^{\prime} / Y^{\prime}\right)\right.$.

On peut donc supposer $z$ (et par suite $T$ ) lisse et $g=f \mid z$ fini surjectif de degré $d$. On a alors $f_{*} z=d z$ et la formule à prouver devient

(4.2.7) $\operatorname{dcl}(T / Y)=f_{*} c l(z / X)$.

On a

$$
\begin{array}{rlrl}
f_{*} c l(z / X) & =f_{*} i_{*}(1) & & (i: z \longrightarrow X) \\
& =j_{*} g_{*}(1) & & (j: T \longrightarrow Y) \\
& =j * g_{*} g^{*}(1) . &
\end{array}
$$

Il suffit alors de voir que $g_{*} g^{*}=d$ car alors $j_{*} g_{*} g^{*}(1)$ $d_{*}(1)=\operatorname{dcl}(T / Y)$.

Proposition 4.2.9. Pour $g: z \longrightarrow T$ fini de degré $d$, on a

(4.2.10) $g_{*} g^{*}=d$ sur $W_{n} \Omega_{T, l o g}^{*} \cdot$

Démonstration : Il suffit de prouver la formule sur $W \Omega_{T}^{*}$ puis d'en prendre les points fixes sous $F$.

La question étant locale sur $T$, on peut supposer que $g$ se relève en un morphisme fini surjectif $g^{\prime}: Z^{\prime} \longrightarrow T^{\prime}$ de degré $d$ de $w_{n}$-schémas lisses. D'après $([1],[27])$ on a

(4.2.11) $9: 9^{\circ}=d$ sur $\Omega_{T}^{*} / w_{n} \cdot$

Comme on le verra ci-dessous, on a $g_{*} g^{*}=\underline{H}^{*}\left(g^{\prime} g^{\prime *}\right)$. D'où le résultat.

Proposition 4.2.12. Soient $Y$ et $Z$ deux sous-schémas fermés intègres de $X$ se coupant proprement (i.e. avec la bonne dimension). on a

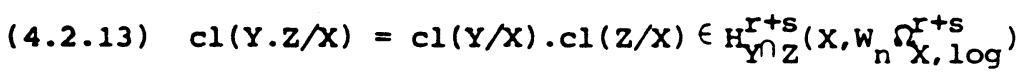


$(r=\operatorname{codim}(Y), s=\operatorname{codim}(Z))$.

Démonstration : Elle comprend plusieurs étapes.

Lemme 4.2.14. Soient $Y$ un sous-schéma fermé lisse de codimension $r$ dans $X$ et $S$ un sous-schéma fermé (non nécessairement lisse) de codimension $q$ dans $Y$. Le cup-produit par $c l(Y / X)$ définit un isomorphisme

$(4.2 .15) \quad \tilde{C}_{Y / X}: H_{S}^{q}\left(Y, W_{n} S_{Y, l o g}^{q}\right) \stackrel{\sim}{\sim} H_{S}^{q+r}\left(X, W_{n} \mathcal{S}_{X, l o g}^{q+r}\right)$.

Démonstration : En effet, on a

$(4.2 .16) \quad \mathrm{H}^{\mathrm{O}}\left(\mathrm{S}-\mathrm{S}_{\text {sing }}, \mathrm{z} / \mathrm{p}^{\mathrm{n}}\right) \stackrel{\sim}{\longrightarrow} \mathrm{H}_{\mathrm{S}}^{\mathrm{q}}\left(\mathrm{Y}, \mathrm{W}_{\mathrm{n}}{ }^{\mathrm{T}} \mathrm{Y}, 10 \mathrm{~g}\right)$

$(4.2 .17) \mathrm{H}^{\mathrm{O}}\left(\mathrm{S}-\mathrm{S}_{\text {sing }}, \mathrm{z} / \mathrm{p}^{\mathrm{n}}\right) \stackrel{\sim}{\longrightarrow} \mathrm{H}_{\mathrm{S}}^{\mathrm{q}+\mathrm{r}}\left(\mathrm{X}, \mathrm{w}_{\mathrm{n}} \mathrm{S}_{\mathrm{X}, \mathrm{log}}^{\mathrm{q}+\mathrm{r}}\right)$

donné par $j_{*}$ (où $j: s-s_{\text {sing }} \longrightarrow Y$ ) et par $j_{*}$ (où $\left.j^{\prime}: S_{\text {sing }} \longrightarrow X\right)$. Le leme résulte alors simplement de la compatibilité du morphisme de Gysin à la composition.

Soit $\Delta: \mathrm{X} \longrightarrow \mathrm{X} \times \mathrm{X}$ le morphisme diagonal. Pour prouver $(4.2 .13)$, il suffit donc de prouver que

$(4.2 .18) \quad \tilde{C}_{\Delta(X) / X \times X}[c l(Y . Z / X)]=\tilde{C}_{\Delta(X) / X 0 X}[c l(Y / X) \cdot c l(Z / X)]$.

Lemme 4.2.19 (réduction à la diagonale). On a

$(4.2 .20) \quad \tilde{C}_{\Delta(X) / X X X}[c l(Y / X) \cdot c l(Z / X)]=c l(Y X Z / X X X) \cdot c l(\Delta(X) / X O X)$

$(4.2 .21) \quad \tilde{C}_{L(X) / X X X}[c l(Y . Z / X)]=c l((Y X Z) . \Delta(X) / X \infty X)$.

Démonstration : On commence tout d'abord par remarquer que $(4.2 .22) \quad c l(Y / X) \otimes c l(Z / X)=c l(Y X Z / X \infty X) \in H_{Y X Z}^{Y+s}\left(X X X, W_{n} \Omega_{X X X, l o g}^{Y+s}\right)$.

En effet, par définition des classes en présence, on peut supposer que $Y$ et $Z$ sont lisses. On utilise alors la description locale des classes en présence (i.e. on remarque que $\otimes$ envoie bien $\operatorname{dlog}\left(\underline{t}_{1}\right) \ldots d \log \left(t_{r}\right), \operatorname{dlog}\left(t_{r+1}\right) \ldots d \log \left(t_{r+s}\right)$ sur $\left.\operatorname{dlog}\left(t_{1}\right) \ldots \operatorname{dlog}\left(t_{r+s}\right)\right)$.

On prend alors les images de $(4.2 .22)$ par $\Delta^{*}$, on obtient $(4.2 .23) \Delta \Delta^{*} \operatorname{cl}(Y X Z / X X X)=c l(Y / X) \cdot c l(Z / X)$. 
M. GROS

D'où

(4.2.24) $\tilde{c}_{\Delta(X) / X 0 X}[c l(Y / X) . c l(Z / X)]=\tilde{c}_{\Delta(X) / X 0 X}\left[\Delta^{*} c l(Y X Z / X X X)\right]=$ $\operatorname{cl}(\mathrm{YXZ} / \mathrm{XXX}) \cdot \operatorname{cl}(\Delta(\mathrm{X}) / \mathrm{X} \times \mathrm{X})$

D'autre part, l'isomorphisme $\Delta: \mathrm{X} \stackrel{\sim}{\longrightarrow}(\mathrm{X})$ fait correspondre $\operatorname{cl}(Y ; Z / X)$ à $\operatorname{cl}\left(\Delta_{*}(Y, Z) / \Delta(X)\right)=\operatorname{cl}((Y X Z) \cdot \Delta(X) / \Delta(X))$. D'où

(4.2.25) $\tilde{C}_{\Delta(X) / X X X}[c l(Y . Z / X)]=c l((Y X Z) . \Delta(X) / \Delta(X)) \cdot c l(\wedge(X) / X X X)=$ $\operatorname{cl}((\mathrm{YXZ}) . \Delta(\mathrm{X}) / \mathrm{X} X \mathrm{X})$.

Pour prouver (4.2.13), on peut donc supposer que (par exemple) $Y$ est lisse. Ecrivant alors

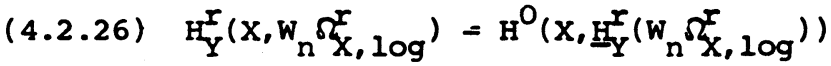

$$
\begin{aligned}
& H_{Z}^{S}\left(X, w_{n} \Omega_{X, \log }^{S}\right)=H^{O}\left(X, H_{Z}^{S}\left(w_{n} S_{X, 10 g}^{S}\right)\right) \text {. }
\end{aligned}
$$

on voit que (pour prouver (4.2.13)) l'on peut se placer au voisinage du point générique $x$ de $(Y \cap \mathrm{Z})_{\text {red }}$.

$D$ 'autre part, en factorisant $Y \longrightarrow X$ en une suite d'immersions fermées de codimension 1 , on voit (par une récurrence évidente) que 1 'on peut supposer $Y$ de codimension 1 .

Le cas le plus simple est celui où $z$ est régulier en $x$, on peut alors supposer que $z$ est défini (au voisinage de $x$ ) par une suite régulière de sections $f_{1} \ldots \ldots f_{S}$ de $\theta_{X} \cdot Z^{\cap} Y$ est alors défini par la suite $f_{1}\left|y, \ldots, f_{s}\right| y$. Par définition, on a

$$
\operatorname{cl}(Y . Z / X)=\left(D_{1}^{\prime} \cdots D_{s}^{\prime}\right) \cdot c l\left((Y . Z)_{r e d} / X\right)
$$

où $D_{i}^{\prime}=\left(E_{i} \mid Y\right)$.

Il s'agit donc de montrer que $d \underline{f}_{1}^{\prime} / \underline{f}_{i}^{\prime} \ldots d \underline{f}_{S}^{\prime} / \underline{f}_{S}^{\prime}$ (avec $f_{i}^{\prime}=f_{i} Y$ ) a la même classe dans $\underline{H}_{X}^{S}\left(W_{n} \mathcal{Z}_{Y, l o g}^{S}\right)$ que $\left(D_{1}^{1} \ldots D_{Y}^{\prime}\right) . c l\left((Y . Z)_{\text {red }} / X\right)$. Ceci résulte de l'égalité

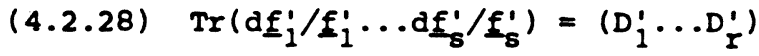

qui sera prouvée ci-dessous.

Le cas général ( $z$ non nécessairement régulier en $x$ ) $n$ 'est pas beaucoup plus difficile. Soit $h: \tilde{z} \longrightarrow z$ la normalisation de $z$ 
(c'est un morphisme fini). Soit $g: P_{X}^{N} \rightarrow X$ le morphisme canonique. On pose $Y^{\prime}=P_{X}^{N} X_{X} Y$. Comme $g$ est lisse, on $a g^{*} c l(Y / X)=$ $c l\left(Y^{\prime} / P_{X}^{N}\right) \cdot D^{\prime}$ où

$$
\begin{aligned}
& \text { (4.2.29) } \operatorname{cl}(\mathrm{Y} / \mathrm{X}) \cdot \operatorname{cl}(\mathrm{Z} / \mathrm{X})=\operatorname{cl}(\mathrm{Y} / \mathrm{X}) \cdot \operatorname{cl}\left(\mathrm{h}_{*} \tilde{\mathrm{Z}} / \mathrm{X}\right) \text { car } \mathrm{h}_{*} \tilde{\mathrm{Z}}=\mathrm{z} \\
& =\operatorname{cl}(\mathrm{Y} / \mathrm{X}) \cdot \mathrm{g}_{*} \operatorname{cl}\left(\tilde{\mathrm{Z}} / \mathrm{P}_{\mathrm{X}}^{\mathrm{N}}\right) \\
& =g_{*}\left(g^{*} c l(Y / X) \cdot c l\left(\tilde{Z} / \mathbb{P}_{X}^{N}\right)\right. \text { (formule de projection) } \\
& =g_{*}\left(c l\left(Y^{\prime} / P_{X}^{N}\right) \cdot c l\left(\tilde{Z} / P_{X}^{N}\right)\right) \text { (lissité de } g \text { ) } \\
& =g_{*}\left(c l\left(Y^{\prime} \cdot \tilde{Z} / P_{X}^{N}\right)\right) \quad \text { (cas précédent) } \\
& =g_{*}\left(\operatorname{cl}\left(g^{*} \mathrm{Y} \cdot \tilde{\mathrm{Z}} / \mathrm{P}_{\mathrm{X}}^{\mathrm{N}}\right)\right) \quad \text { (définition de } \mathrm{Y}^{\prime} \text { ) } \\
& =\operatorname{cl}\left(g_{*}\left(g^{*} \mathrm{Y} \cdot \tilde{\mathrm{Z}}\right) / \mathrm{X}\right) \quad \text { (proposition 4.2.3) } \\
& =\operatorname{cl}\left(g_{*} \tilde{Z} \cdot Y / X\right) \quad \text { (formule de projection) } \\
& =\operatorname{cl}(\mathrm{Y} \cdot \mathrm{Z} / \mathrm{X}) \text {. }
\end{aligned}
$$

Pour vérifier $(4.2 .28)$, on remarque que le morphisme trace

$$
\text { (4.2.30) } \operatorname{Tr}_{f, y}: f_{n * H_{y}^{N}}\left(w_{n} \delta \delta_{x}^{N}\right) \rightarrow w_{n} \quad N=\operatorname{dim}(x)
$$

( $y$ un point fermé de $x, x$ un $k$-schéma lisse, $f_{n}$ le morphisme canonique $\left.w_{n} x=\left(|x|, w_{n} x\right) \longrightarrow w_{n}\right)$ défini par Ekedahl $([9])$ est lié au symbole résidu (défini également par Ekedahl ([10])) par le

Lemme 4.2.31. Pour tout $\omega \in w_{n} S_{X, Y}^{N}$ et tout entier $j$, on a

$$
\text { (4.2.32) } \operatorname{Tr}_{f, y}\left(\omega / t_{1}^{j} \ldots t_{N}^{j}\right)=\operatorname{Res} x / w_{n}\left[\begin{array}{l}
\omega \\
t_{1}^{j}, \ldots, t_{N}^{j}
\end{array}\right]
$$

où $t_{1}, \ldots, t_{N}$ est une suite régulière de sections de $\theta_{X}$ définissant $Y$. Nous omettrons la démonstration de ce lenme, essentiellement analogue à celle du cas $n=1$. (cf. [2]).

On en déduit (cf. la formule R6 p. 198 de [17]) (4.2.28).

Proposition 4.2.33. Supposons $k$ algébriquement clos. Deux cycles algébriquement équivalents de codimension $r$ ont même classe de cohomologie dans $\mathrm{H}^{\mathrm{r}}\left(\mathrm{X}, \mathrm{w}_{\mathrm{n}}{ }^{\mathrm{r}} \mathrm{X}, \log \right)$. 
Démonstration $^{(*)}$ : Précisons tout d'abord ce que nous entendrons par cycles algébriquement équivalents. Sojent $C$ une courbe propre et lisse sur $k$ et $z$ un sous-schéma fermé de $x x_{k} c$ de codimension $r+1$. Alors deux cycles seront dits algébriquement équivalents s'ils sont images réciproques dans. $\mathrm{z}$ de deux points de la courbe $\mathrm{C}$ pour un $\mathrm{z}$ convenablement choisi.

Soit donc un $\mathrm{z}$ comme ci-dessus, on dispose d'un morphisme

$$
\text { (4.2.34) } \operatorname{Div}(C) \longrightarrow \mathrm{H}^{r}\left(\mathrm{x}, \mathrm{w}_{\mathrm{n}} \Omega^{\mathrm{r}} \mathrm{x}, 10 \mathrm{~g}^{2}\right)
$$

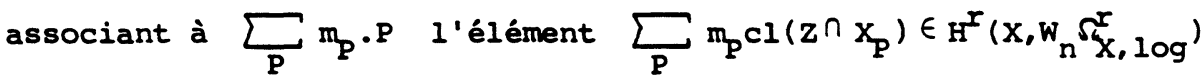

$\left(\mathrm{X}_{\mathrm{p}}\right.$ désignant $\mathrm{X} \mathrm{x}_{\mathrm{k}}\{\mathrm{P}\}$ identifié à $\mathrm{X}$ ).

on va voir que $(4.2 .34)$ se factorise en un morphisme

$$
\text { (4.2.35) Pic(C) } \longrightarrow \mathrm{H}^{\mathrm{Y}}\left(\mathrm{X}, \mathrm{w}_{\mathrm{n}} \Omega \mathrm{X}_{\mathrm{X}}^{\mathrm{r}} \log \right) \text {. }
$$

Deux éléments linéairement équivalents de $\operatorname{Div}(C)$ proviennent par image réciproque par un morphisme $g: C \longrightarrow \mathbf{P}_{k}^{1}$ de deux points 0 et $\infty$ de $\mathbf{P}_{k}^{1}$. Utilisant le morphisme composé $\mathrm{z} \longrightarrow \mathrm{Xx}_{\mathrm{k}} \mathrm{C} \longrightarrow$ $c \longrightarrow \mathbf{P}_{k}^{1}$, on voit donc que $l^{\prime}$ on est ramené à voir que deux cycles rationnellementéquivalents de $X$ ont même classe fondamentale de cohomologie. On peut donc supposer $C=P_{k}^{1}$.

D'après $I(2.1 .16)$, on dispose d'un isomorphisme

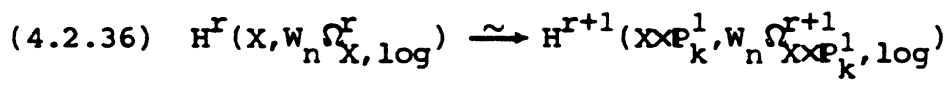

donné par le produit par $c_{1}\left(\theta_{\mathbf{P}_{k}^{1}}(1)\right) \in \mathrm{H}^{1}\left(\mathbf{P}_{\mathrm{k}}^{1}, \mathrm{w}_{n} \Omega_{\mathbf{p}_{k}^{1}}^{1}, \log \right)$.

Si $Y_{1}$ et $Y_{2}$ désignent deux cycles rationnellement équivalents dont on veut comparer les classes de cohomologie, il suffit d'après ce qui précède de comparer les classes de $Y_{1} \times\{0\}$ et $Y_{2} \times\{\infty\}$ dans $H^{r+1}\left(x<P_{k}^{1}, w_{n} \Omega^{r+1} \times P_{k}^{1}, \log \right)$.

D'autre part, $\mathrm{z}$ rencontre $\mathrm{x} \times\{0\}$ et $\mathrm{x} \times\{\infty\}$ proprement.

La formule $(4.2 .4)$ donne alors

(*) D'après un argument da (indépendamment) à Berthelot et Suslin. 
$(4.2 .37)$

$$
\begin{aligned}
& c l\left(Y_{1} \times\{0\} / X \times P_{k}^{1}\right)=c l\left(Z / X \times P_{k}^{1}\right) \cdot c l\left(X \times\{0\} / X \times P_{k}^{1}\right) \\
& c l\left(Y_{2} \times\{\infty) / X \times P_{k}^{1}\right)=c l\left(Z / X \times \mathbb{P}_{k}^{1}\right) \cdot c l\left(X \times\{\infty\} / X \times P_{k}^{1}\right)
\end{aligned}
$$

d'où l'égalité puisque $\{0\}$ et $\{\infty\}$ ónt même classe de cohomologie. Soient alors $\mathrm{PiC}^{\mathrm{O}}(\mathrm{C})$ la Jacobienne de $\mathrm{C}$ et $\mathrm{NS}(\mathrm{C})=\operatorname{Pic}(\mathrm{C}) / \mathrm{PiC}^{\mathrm{O}}(\mathrm{C})$ le groupe de Néron-Sévéri de $C$. On va montrer que (4.2.35) se factorise par un morphisme (4.2.38) NS (C) $\longrightarrow \mathrm{H}^{r}\left(\mathrm{X}, \mathrm{w}_{\mathrm{n}} \Omega_{\mathrm{X}, \mathrm{x}, \log }^{\mathrm{r}}\right)$ On remarque simplement que $\mathrm{Pic}^{\circ}(\mathrm{C})$ est un groupe $\mathrm{p}$-divisible et que par conséquent $I^{\prime}$ image de $\operatorname{Pic}^{0}(C)$ dans $H^{r}\left(x, w_{n} \Omega_{x, l o g}^{r}\right.$ par (4.2.7) est contenu dans $\bigcap_{m} p^{m_{H} r}\left(x, w_{n} \Omega_{x}^{r}, l o g\right)$ et est donc nulle puisque $p^{m_{H} r}\left(x, w_{n} \Omega_{x}^{x}, g^{2}\right)=0$ pour $m \geqslant n$. D'où $(4.2 .38)$.

Deux cycles algébriquement équivalents de $\mathrm{X}$ provenant de points de $\mathrm{C}$ ont donc même classe de cohomologie.

Corollaire 4.2.39. Deux cycles algébriquement équivalents ont même classe de cohomologie cristalline.

\section{Morphisme de Gysin et complexe de Cousin.}

\subsection{Cas de la cohomologie de Hodge-Witt.}

Reprenons la situation du numéro 1.2 , on va voir que le morphisme 1.2 .6 peut se réaliser comme un morphisme entre complexes de Cousin. Rappelons que le complexe de Cousin d'un faisceau abélien $F$ est le complexe Cous(F)

$$
\begin{aligned}
& \text { (5.1.1) } \quad 0 \longrightarrow F \longrightarrow \underset{x}{\oplus} i_{* x}{ }_{x}^{H}(F) \longrightarrow \underset{x}{\oplus}{ }^{H} \cdot{ }_{x}^{H}{ }_{x}^{1}(F) \\
& \stackrel{\oplus}{x}+{ }_{x} \stackrel{H}{x}_{x}^{a}(F) \longrightarrow 0
\end{aligned}
$$

où les sommes sont prises sur les points de codimension $0, \ldots, \mathbf{a}=\operatorname{dim}(X)$.

Proposition 5.1.2. Le complexe de cousin de $w_{n} \Omega_{x}$ est une résolution flasque de $w_{n} R^{*}$. 
M. GROS

Démonstration : La proposition résulte de $(3.3 .20)$ et de ([17, IV 2.6]).

On va voir que le morphisme de Gysin

$$
f_{*}: \operatorname{Rf}_{*} W_{n} \Omega_{Y} \longrightarrow W_{n} R_{X}^{*}(r)[r]
$$

se réalise comme un morphisme de bicomplexes

$$
(5.1 .4) \quad f_{*}: f_{*} \operatorname{Cous}\left(w_{n} \Omega \dot{Y}\right) \longrightarrow \operatorname{Cous}\left(w_{n} \Omega(r)\right)[r] .
$$

Proposition 5.1.5. On a un isomorphisme canonique

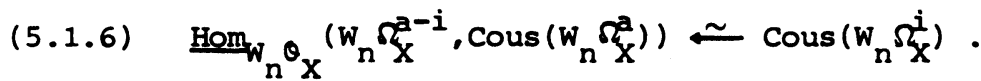

Démonstration : on a un isomorphisme canonique (cf. $[9$, II (2.2.1)])

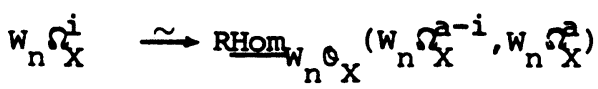

d'où un isomorphisme

$$
\text { (5.1.8) } \quad R \Gamma_{x}\left(w_{n} \Omega_{x}^{i}\right) \stackrel{R H O m_{w}}{\sim}{ }_{n}\left(w_{n} S_{x}^{2-i}, R \Gamma_{x}\left(w_{n} \Omega_{x}^{2}\right)\right)
$$

pour tout point $x$ de codimension $j \in[0, a]$.

Chacun des foncteurs $R \Gamma_{x}(-)$ et ${\mathbb{R H O m _ { W }}}_{n} \theta_{X}\left(w_{n} \Omega_{x}^{a-i},-\right)$ n'a (sur les objets auxquels ils s'appliquent) qu'un seul objet de cohomologie non nul, on déduit donc de (5.1.8) un isomorphisme

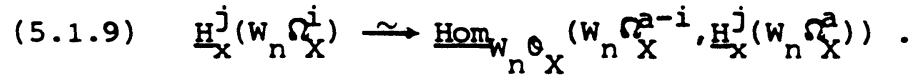

(La nullité des $\operatorname{Ext}_{w_{n} \theta_{X}}^{i}\left(w_{n} \Omega^{a-*} \cdot{ }_{x}^{H_{x}^{j}}\left(w_{n} V_{x}^{a}\right)\right.$ ) résultant de la nullité des $\operatorname{Ext}_{w_{n} \theta_{x}}^{i}\left(w_{n} v^{a-*}, w_{n} r^{a}\right)$ pour $i>0$ et de la description des groupes de cohomologie à support donnée en (3.3.6)).

Il en résulte facilement l'isomorphisme (5.1.6).

On remarque alors que, $w_{n} r_{x}^{g}$ étant un complexe dualisant, le morphisme trace $(1.1 .2)$ se réalise à l'aide de complexes résiduels (cf. [17]) i.e. comme un morphisme

$$
(5.1 .10) f_{0} \operatorname{Cous}\left(w_{n} R_{Y}^{b}\right)[b] \rightarrow \operatorname{cous}\left(w_{n} \Omega_{X}^{a}\right)[a] .
$$

Soit $x \in f_{\text {. Cous }}\left(w_{n} \Omega_{Y}^{\dot{i}}\right), x$ définit (grâce à $(5.1 .7)$ ) un élément

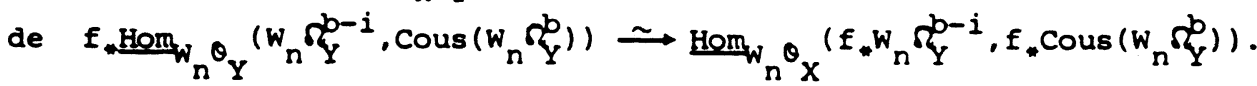
Utilisant le morphisme de fonctorialité 


$$
w_{n} r^{b-i} \longrightarrow f_{*} w_{n} r^{b-i}
$$

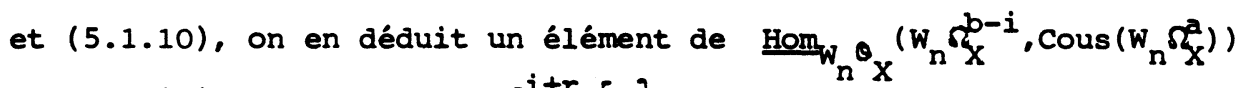
i.e. un élément de $\operatorname{cous}\left(w_{n} \Omega^{i+r}\right)[r]$. C'est l'image de $x$ par $(5.1 .5)$.

On va maintenant vérifier la compatibilité de $(5.1 .5)$ à $d$. La vérification est de nature locale au voisinage d'un point $x$ de codimension $m$ de $\mathrm{x} \cdot \mathrm{w}_{\mathrm{n}} \mathrm{r}^{\mathrm{a}}[\mathrm{a}]$ est un complexe dualisant normalisé, on peut donc appliquer $([17, \mathrm{~V} 6.5])$ pour obtenir l'isomorphisme fonctoriel de dualité locale

$$
\text { (5.1.12) }\left(w_{n} \Omega_{x}^{i}\right) \hat{x} \stackrel{\text { Hom }}{\sim}\left(\underline{H}_{x}^{m}\left(w_{n} \Omega_{x}^{2-i}\right), I\right)
$$

où I est l'enveloppe injective du corps résiduel de $x$ et où n désigne la complétion en $x$.

Soit maintenant $y \in Y$ tel que $f(y)=x$, on a de même un isomorphisme

$$
\left(W_{n} \Omega_{Y}^{i}\right)_{Y}^{\wedge} \underset{\operatorname{Hom}}{\sim}\left(\underline{H}_{Y}^{m+r}\left(W_{n} \Omega_{Y}^{b-i}\right), I^{\prime}\right)
$$

où I' désigne l'enveloppe injective du corps résiduel de $Y$.

On est ainsi ramené à voir la commutativité du diagramme

$$
\begin{gathered}
f_{*}\left(w_{n} \Omega_{y}^{i}\right)_{y} \longrightarrow f_{*}\left(w_{n} \Omega_{y}^{i+1}\right)_{y} \\
\left.\operatorname{adj}\right|^{\dagger a d j} \\
\left(w_{n} \delta_{x}^{i}\right)_{x} \longrightarrow d
\end{gathered}
$$

qui est claire.

\subsection{Cas de la cohomologie logarithmique.}

J'ignore si l'on peut réaliser le morphisme $f_{*}$ : $R_{*} W_{n} \Omega_{Y, l o g}^{*} \longrightarrow w_{n} \Omega_{X, \log }^{*}(r)[r]$ à $I^{\prime a i d e}$ des points fixes par $F$ du complexe de cousin de $w_{n} \Omega_{\dot{x}}$.

On remarquera simplement que Milne construit pour $x$ une surface une résolution flasque (pour la topologie étale sur $x$ ) de $w_{n} \delta^{2}, l o g$ qui se déduit de la résolution de Cousin de $w_{n} r_{x}^{2}$ en prenant les points fixes sous $F$ (cf. [29]). 
CHAPITRE III

CLASSES DE CHERN LOGARITHMIQUES

Dans tout ce chapitre, les $k$-schémas considérés sont supposés lisses.

\section{Classes de Chern logarithmiques.}

\subsection{Enoncé du théorème principal.}

Théorème 1.1.1. Soit $n$ un entier $\geqslant 1$, il existe une unique theorie des classes de Chern associant à tout $\theta_{x}$-Module localement libre de rang $r+1(r \geqslant-1)$ des classes de Chern $c_{i}(E) \in H^{i}\left(x, w_{n} \delta_{x, l o g}^{i}\right)$, $i \in \mathbf{N}$, satisfaisant aur conditions suivantes

(1.1.2) (Fonctorialité) Pour tout morphisme de $k$-schémas . lisses $f: Y \rightarrow X$, on a $c_{i}\left(f^{*} E\right)=f^{*} c_{i}(E)$ pour tout $i \in[0, r+1]$.

(1.1.3) (Normalisation) $\mathrm{Si} E$ est inversible, $\mathrm{C}_{\mathrm{O}}(E)=1$ et $\mathrm{C}_{1}(\mathrm{E})$ est l'élément de $H^{1}\left(x, w_{n} \Omega_{X}^{1}, l o g\right)$ image de la classe de $E$ dans Pic(X) par le morphisme induit en cohomologie par dlog $: \theta_{x}^{*} \longrightarrow w_{n} \Omega_{x, l o g}^{1}$

(1.1.4) (Additivité) Pour toute suite exacte de ${ }^{\theta} x^{\text {-Modules }}$ localement libres de type fini

$$
\mathrm{O} \longrightarrow \mathrm{E}^{\prime} \longrightarrow \mathrm{E} \longrightarrow \mathrm{E}^{\prime \prime} \longrightarrow \mathrm{O}
$$

et tout $i$, on a $c_{i}(E)=\sum_{h+m=i} c_{h}\left(E^{\prime}\right) c_{m}\left(E^{\prime \prime}\right)$.

\subsection{Démonstration du théorème.}

Prouvons l'unicité d'une théorie de classes de Chern vérifiant $(1.1 .2),(1.1 .3),(1.1 .4)$. Soit donc $E$ un $\theta_{x}$-Module localement libre de rang $r+1$ et soit $D$ le fibré en drapeaux de $E$ muni de sa projection $f: D \longrightarrow X$. Comme $f$ est composé de fibrations projectives, le corollaire 2.1 .15 de I montre que le morphisme $f^{*}: H^{i}\left(x, w_{n} \Omega^{i}, l o g\right) \rightarrow H^{i}\left(D, w_{n} \Omega_{D}^{i}, l o g\right)$ est injectif pour tout $i \geqslant 0$. 
La classe $c_{i}(E)$ est donc connue lorsque l'on connait $f^{*} c_{i}(E)$, c'est-à-dire grâce à (1.1.2) $c_{i}\left(f^{*} E\right)$. Or $f^{*} E$ est muni d'une suite de composition $\left(E_{j}\right)_{0} \leqslant j \leqslant r+1$ avec $\operatorname{rang}\left(E_{j}\right)=r+1-j$. Par récurrence sur $r$, on voit alors grâce à $(1.1 .4)$ que

$$
c_{i}\left(f^{*} E\right)=\sigma_{i}\left(c_{1}\left(E_{0} / E_{1}\right), \ldots, c_{1}\left(E_{r} / E_{r+1}\right)\right)
$$

où $\sigma_{i}$ désigne la $i$-ème fonction symétrique élémentaire.

Comme $E_{j} / E_{j+1}$ est inversible pour tout $j \in[0, r]$, on connait grâce à (1.1.3) $\quad c_{1}\left(E_{j} / E_{j+1}\right)$ pour tout $j \in[0, r]$. D'où le résultat.

Définissons maintenant les classes $c_{i}(E)$. La décomposition (2.1.16) de I appliquée au fibré projectif $P=P(\stackrel{E}{)}$ fournit donc un isomorphisme

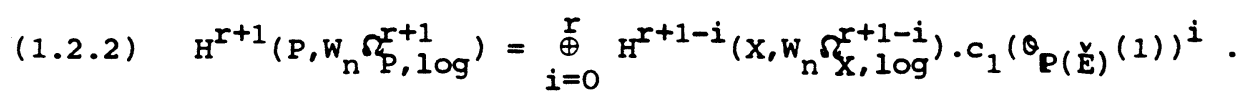

Appliquant cette décomposition à $c_{1}\left(\theta_{P(L)}(1)\right)^{r+1} \in H^{r+1}\left(P, W_{n} \delta_{P}^{r+1} \log \right)$, on voit que $l$ 'on peut trouver de manière unique des éléments $c_{i}(E) \in H^{i}\left(X, W_{n} \Omega^{i}, \log \right)$ satisfaisant aux conditions suivantes

$$
\begin{aligned}
& c_{0}(E)=1, c_{i}(E)=0 \text { si } i>r+1, \\
& \sum_{i=0}^{r+1} c_{i}(E) c_{1}\left(\theta_{P(E)}(1)\right)^{r+1-i}=0 .
\end{aligned}
$$

Les classes $c_{i}(E)$ seront appelées classes de Chern (Logarithmiques) de $E$.

Fonctorialité : Soit $F=f^{\star} E$, le fibré projectif associé à noté $P(\breve{F})$ est l'image réciproque du fibré projectif associé à et noté $\mathbf{P}(\grave{E})$. On a donc

$$
\text { (1.2.4) } \quad c_{1}\left(\theta_{P(L)}(1)\right)=f^{*} c_{1}\left(\theta_{P(E)}(1)\right) .
$$

L'égalité de $(1.1 .2)$ est trivialement vérifiée pour $i=0$ et $i) r+1$, vérifions là pour $i \in[1, r+1]$. Par définition, on a (1.2.5) $\quad \sum_{i=0}^{r+1} q^{*}\left(c_{i}(E)\right) c_{1}\left(\theta_{P(E)}(1)\right)^{r+1-i}=0$ où $q$ désigne la projection $P(\check{E}) \longrightarrow X$. Soit $q^{\prime}$ la projection $P(\check{F}) \longrightarrow Y$, on a $(1.2 .6) \quad f^{*} q^{*}=q^{\prime} f^{*}$. 
M. GROS

Appliquant $f^{*}$ à l'égalité (1.2.5), on obtient

(1.2.7) $\sum_{i=0}^{F+1} q^{\prime}{ }^{*} f^{*} c_{i}(E) c_{1}\left(\theta_{P(F)}(1)\right)^{r+1-i}=0$.

ce qui par définition prouve que

(1.2.8) $\quad c_{i}(F)=f^{*} c_{i}(E)$ pour $i \in[1, r+1]$.

D'où la fonctorialité.

Normalisation : Supposons $E$ inversible, on a alors un isomorphisme

(1.2.9) $E \stackrel{\sim}{\longrightarrow} \theta_{P(E)}(1)$

d'où

(1.2.10) $\operatorname{dlog}([E])=\operatorname{dlog}\left(\left[\theta_{\mathbf{P}(E)}(1)\right]\right)$

où [ ] désigne la classe dans $\mathrm{Pic}(\mathrm{X})$.

D'autre part, la définition des classes de Chern montre que $l$ 'on doit avoir

(1.2.11) $c_{1}(E)+\operatorname{dlog}\left(\theta_{\mathbf{P}(\check{E})}(1)\right)=0$.

D'où le résultat.

Additivité : Nous aurons besoin du lemme suivant

Lemme 1.2.12. Soit $E$ un $\theta_{x}$-Module localement libre muni d'une filtration finie $O=E_{O} \longrightarrow E_{1} \longrightarrow \ldots \longrightarrow E_{F+1}=E$ dont les quotients consécutifs $L_{i}=E_{i} / E_{i-1} \quad(i \in[1, r+1])$ sont inversibles.

Alors on a

$(1.2 .13) \quad c_{j}(E)=\sigma_{j}\left(c_{1}\left(L_{1}\right), \ldots, c_{1}\left(L_{r+1}\right)\right)$

$\left(\sigma_{j}\right.$ désignant la j-ième fonction symétrique élémentaire).

Montrons comment 1.2 .12 entraine (1.1.4).

Soit $h: z=D^{\prime} \times D^{\prime \prime} \longrightarrow X$ le produit fibré des schémas de drapeaux $D^{\prime}$ et $D^{\prime \prime}$ de $E^{\prime}$ et $E^{\prime \prime}$ respectivement. Alors $h^{\prime \prime} E^{\prime}$ et $h " E$ " admettent des filtrations finies à quotients consécutifs inversibles $\left(L_{i}^{\prime}\right)_{1} \leqslant i \leqslant r^{\prime}+1$ et $\left(L_{j}^{\prime \prime}\right)_{1} \leqslant j \leqslant r^{\prime \prime}+1$ respectivement $\left(I^{\prime}=\operatorname{rang}\left(E^{\prime}\right)\right.$ et $\left.I^{\prime \prime}=\operatorname{rang}\left(E^{\prime \prime}\right)\right)$. Il en résulte en particulier que 
$h^{*} \mathrm{E}$ admet une filtration à quotients consécutifs les $L_{i}^{\prime}$ et les $L_{j}^{\prime \prime}$. L'application de 1.2.12 montre alors que

$$
c_{j}\left(h^{*} E\right)=\sigma_{j}\left(c_{1}\left(L_{1}^{\prime}\right), \ldots, c_{1}\left(L_{r}^{\prime}\right), c_{1}\left(L_{1}^{\prime \prime}\right), \ldots, c_{1}\left(L_{r^{\prime \prime}}^{\prime \prime}\right)\right) .
$$

Comme

$$
\begin{array}{r}
\sigma_{j}\left(x_{1}, \ldots, x_{r^{\prime}+1}, t_{1}, \ldots, t_{r^{\prime \prime}+1}\right)=\sum_{n+m=j} \sigma\left(x_{1}, \ldots, x_{r^{\prime}+1}\right) \\
\sigma_{m}\left(t_{1}, \ldots, t_{r^{\prime \prime}+1}\right)
\end{array}
$$

on en déduit que

$$
(1.2 .16) \quad c_{j}\left(h^{*} E\right)=\sum_{h+m=j} c_{h}\left(h^{*} E^{\prime}\right) c_{m}\left(h^{*} E^{\prime \prime}\right) \text {. }
$$

D'autre part, comme on l'a déjà remarqué, $h^{*}$ est injectif, on en déduit donc (grâce à la fonctorialité (1.1.2)) que

$$
\text { (1.2.17) } \quad c_{j}(E)=\sum_{h+m=j} c_{h}\left(E^{\prime}\right) c_{m}\left(E^{\prime \prime}\right) \text {. }
$$

Venons-en à la démonstration de 1.2.12.

Soit $f$ la projection $P=P($ E $) \longrightarrow X$. Il résulte de I 2.1 .15 que

$(1.2 .18) f^{*}: H^{i}\left(x, w_{n} \Omega_{x, l o g}^{i}\right) \longrightarrow H^{i}\left(P, w_{n} \Omega_{P, l o g}^{i}\right)$

est injectif pour tout $i \geqslant 0$.

Il suffit donc de prouver que

$(1.2 .19) \quad f^{*} c_{j}(E)=f^{*} \sigma_{j}\left(c_{1}\left(L_{1}\right), \ldots, c_{1}\left(L_{r+1}\right)\right)$

soit encore, utilisant la fonctorialité (1.1.2)

$$
c_{j}\left(f^{*} E\right)=\sigma_{j}\left(c_{1}\left(f^{*} L_{1}\right), \ldots, c_{1}\left(f^{*} L_{r}\right)\right) .
$$

Pour tout $i \in[1, r+1]$, l'inclusion $E_{i} \longrightarrow E$ définit un épimorphisme $E \rightarrow E_{i}$. On posera $v_{i}=P\left(\check{E}_{i}\right)$. On obtient ainsi une suite de sous schémas fermés de $P$

$$
(1.2 .21) \quad X \stackrel{\longrightarrow}{\longrightarrow} Y_{1} \longrightarrow \ldots \longrightarrow Y_{i} \longrightarrow \ldots \longrightarrow Y_{Y+1}=P \text {. }
$$

On notera ${ }^{\alpha} j, i \quad l$ 'immersion fermée canonique $Y_{i} \longrightarrow Y_{j}$ $(1 \leqslant i \leqslant j \leqslant r+1)$. Il résulte alors du lemme ci-dessous que $Y_{i}$ est défini dans $Y_{i+1}$ par un idéal inversible isomorphe à 
M. GROS

$(1.2 .22) \quad \alpha_{r+1, i+1}^{*}\left(f^{*}\left(L_{i+1}^{v}\right) \otimes \theta_{P}(-1)\right)$.

Lemme 1.2.23 (SGA 5, exp. VII, Lemme 3.1.6). Soit

$\mathrm{O} \longrightarrow \mathrm{I} \longrightarrow \mathrm{E} \longrightarrow \mathrm{F} \longrightarrow \mathrm{O}$ une suite exacte de $\theta_{X}$-Modules 1ocalement libres. Alors la suite $0 \longrightarrow L^{\otimes}{ }_{\theta_{X}} S(E) \longrightarrow \vec{u} S(E) \rightarrow V(F) \longrightarrow 0$ dans laquelle $\mathrm{v}$ désigne le morphisme évident et $u$ le morphisme de degré 1 correspondant à l'inclusion de $I$ dans $E$ est exacte.

On va alors montrer par récurrence croissante sur $i$ que

(1.2.24) $\left(\alpha_{r+1, i}\right)^{*}\left(\alpha_{r+1, i}\right)^{*}\left(\prod_{j \leqslant i} c_{1}\left(f^{*}\left(L_{j}\right) \otimes \theta_{P}(-1)\right)=0\right.$.

Dans le cas $i=r+1,(1.2 .24)$ s'écrit

$(1.2 .25) \prod_{i} c_{1}\left(\left(f^{*} L_{i}^{v}\right) \otimes \theta_{p}(-1)\right)=0$

soit encore

$$
\text { (1.2.26) } \prod_{i}\left(c_{1}\left(f^{*} L_{i}^{v}\right)+c_{1}\left(\theta_{P}(-1)\right)\right)=0 \text {. c }
$$

En développant cette égalité, on voit (par définition des $\left.c_{i}(E)\right)$ que les $c_{i}(E)$ sont les fonctions symétriques élémentaires en les $c_{1}\left(L_{i}\right)$.

Prouvons donc $(1.2 .24)$. Pour $i=1$, on remarque que, $Y_{1}$ s'identifiant à $\mathrm{X}$, on a un isomorphisme

$(1.2 .27) \quad \alpha_{I+1,1}^{*}\left(f^{*} L_{1}^{v}\right) \stackrel{\sim}{\longrightarrow} \theta_{Y_{1}}(1)$

soit

$(1.2 .28) \quad \alpha_{T+1,1}^{*}\left(f^{*}\left(L_{1}^{\nu}\right) \otimes \theta_{P}(-1)\right) \stackrel{\sim}{\longrightarrow} \theta_{Y_{1}} \stackrel{\sim}{\longrightarrow} \theta_{X}$.

d'où l'assertion $(1.2 .24)$ dans ce cas.

Supposons (1.2.24) prouvé pour $i$ et prouvons là pour $i+1$. On posera $N=\prod_{j \leqslant i} c_{1}\left(f^{*}\left(L_{j}^{v}\right) \otimes \theta_{P}(-1)\right)$. L'égalité $(1.2 .24)$ pour $i$ s'écrit ( $\left.\operatorname{car} \alpha_{r+1, i}=\alpha_{i+1, i}{ }^{\circ \alpha}{ }_{r+1, i+1}\right)$

$(1.2 .29) \quad\left(\alpha_{r+1, i+1}\right) \cdot\left(\alpha_{i+1, i}\right) \cdot\left(\alpha_{i+1, i}\right) *\left(\alpha_{r+1, i+1}\right)^{*}(N)=0$.

Utilisant la formule de projection II (2.2.6) pour $\alpha_{i+1, i}$ et la compatibilité entre classe de Chern et classe de cycle II (2.5.2) pour $\alpha_{i+1, i}\left(Y_{i}\right.$ étant défini dans $Y_{i+1}$ par l'idéal inversible $(1.2 .22))$, on obtient 
$(1 \cdot 2 \cdot 30) \quad\left(\alpha_{r+1, i+1}\right)_{*}\left(\left(\alpha_{r+1, i+1}\right)^{*}(N) \cdot\left(\alpha_{r+1, i+1}\right)^{*} c_{1}\left(f^{*} L_{i+1}^{\nu} \otimes \theta_{P}(-1)\right)\right)=0$.

D'où le résultat.

Ce qui termine la démonstration du théorème 1.1 .1$.

Remarquons que cette démonstration est (une fois établi les résultats et compatibilités concernant le morphisme de Gysin) entièrement formelle et analogue à celle donnée dans [13].

Corollaire 1.2.31 (i) Les classes de Chern d'un produit tensoriel ou d'une puissance extérieure s'expriment au moyen des polynômes universels explicités dans [SGA 6, 6.1].

(ii) En particulier, si $\mathrm{E}$ est un $\theta_{\mathrm{X}}$-Module localement libre de rang $r+1$, on a $c_{1}(E)=c_{1}\left(\Lambda^{r+1} E\right)$.

(iii) Pour tout entier $i$ et tout $\theta_{x}$-Module localement libre $E$, on a $c_{i}(\stackrel{v}{E})=(-1)^{i} c_{i}(E)$.

Démonstration : Une fois utilisé le "splitting-principle", la démonstration est entièrement formelle (cf. SGA 5, VII, 3.7).

\section{Variantes et compatibilités.}

\subsection{Comparaison avec les classes de Chern cristallines.}

Le morphisme d'injection canonique $w_{n} \Omega_{x, \log }^{(-i)} \rightarrow w_{n} \Omega_{x}$ induit en cohomologie un morphisme

$$
\text { (2.1.1). } \mathrm{H}^{i}\left(\mathrm{x}, \mathrm{w}_{\mathrm{n}} \Omega_{\mathrm{X}, \log }^{\mathrm{i}}\right) \longrightarrow \mathrm{H}^{2 i}\left(\mathrm{x}, \mathrm{w}_{\mathrm{n}} \Omega_{\mathrm{X}}\right) \stackrel{\sim}{\longrightarrow} \mathrm{H}_{\mathrm{cris}}^{2 \mathrm{i}}\left(\mathrm{x} / \mathrm{w}_{\mathrm{n}}\right) .
$$

L'image des classes $c_{i}(E) \in H^{i}\left(x, W_{n} \Omega^{i}, l \circ g\right)$ dans $H_{c r i s}^{2 i}\left(x / W_{n}\right)$ sera encore notée $c_{i}(E)$.

D'autre part, Berthelot et Illusie ont défini dans [4] des classes de Chern $\underline{c}_{i}(E) \in \mathrm{H}_{c r i s}^{2 i}\left(X / W_{n}\right)$ pour tout $\theta_{X}$-Module localement libre de type fini $E$. Il y a donc lieu de comparer ces classes $c_{i}(E)$ avec les classes $c_{i}(E)$.

L'isomorphisme I (5.1.3) fournit une décomposition canonique

$$
\text { (2.1.2) } \quad H_{c r i s}^{j}\left(P / w_{n}\right)=\underset{i=0}{\bigoplus} H_{c r i s}^{j-2 i}\left(x / w_{n}\right) \cdot \varepsilon_{1}\left(\theta_{p}(1)\right)^{i}
$$


M. GROS

où $P=P(\stackrel{E}{E})$ est le fibré projectif associé au dual d'un $\theta_{X}$-Module localement libre de rang $r+1$.

Comme I'isomorphisme

$$
\mathrm{H}_{\mathrm{Cris}}^{j}\left(\mathrm{x} / \mathrm{w}_{\mathrm{n}}\right) \stackrel{\sim}{\longrightarrow} \mathrm{H}^{j}\left(\mathrm{X}, \mathrm{w}_{\mathrm{n}} \mathrm{R} \dot{\mathrm{x}}\right)
$$

induit par l'isomorphisme canonique de comparaison (Thm. 1.4 de [21])

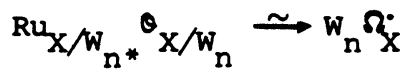

est fonctoriel, pour voir que le diagramme suivant est commutatif

$$
\begin{aligned}
& \mathrm{H}_{\text {cris }}^{j}\left(\mathrm{P} / \mathrm{W}_{\mathrm{n}}\right) \longrightarrow \mathrm{H}^{\mathrm{j}}\left(\mathrm{P}, \mathrm{W}_{\mathrm{n}} \quad \dot{\mathrm{P}}\right) \\
& \underset{i=0}{\stackrel{r}{\oplus}} \mathrm{H}_{\operatorname{cris}}^{j-2 i}\left(x / w_{n}\right) \cdot \underline{c}_{1}\left(\theta_{P}(1)\right)^{i} \underset{i=0}{\sim} \underset{\oplus}{\longrightarrow} H^{j-2 i}\left(x, w_{n} \Omega_{X}^{j}\right) \cdot c_{1}\left(\theta_{P}(1)\right)^{i}
\end{aligned}
$$

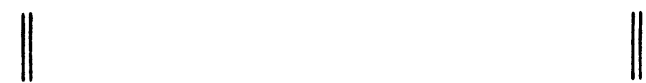

il suffit de voir que $\underline{c}_{1}\left(\theta_{p}(1)\right)$ a pour image $c_{1}\left(\theta_{p}(1)\right)$ dans $\mathrm{H}_{\text {cris }}^{2}\left(\mathrm{P} / \mathrm{W}_{\mathrm{n}}\right)$ ce qui a été vu au numéro 5 de $\mathrm{I}$.

Comme les $\underline{c}_{i}(E)$ sont obtenues par la relation

$$
\begin{aligned}
& c_{0}(E)=1, c_{i}(E)=0 \text { pour } i>r+1, \\
& \sum_{i=0}^{F+1} \varepsilon_{i}(E) c_{1}\left(\theta_{P(E)}(1)\right)^{r+1-i}=0 .
\end{aligned}
$$

On voit que $l^{\prime}$ on a bien coIncidence entre $c_{i}(E)$ et $c_{i}(E)$.

Remarque. On prendra garde au fait que dans [4], le théorème de structure des fibrés projectifs est appliqué à $P=P(E)$ et que par suite, il s'introduit des signes dans la décomposition (2.1.2) si l'on veut obtenir la coincidence entre $c_{i}(E)$ et $c_{i}(E)$.

\subsection{Cohomologie d'un schéma de drapeaux.}

Soit $E$ un $\theta_{x}$-Module localement libre de rang $r+1$. Soit d'autre part, une suite $\left(p_{1}, \ldots, p_{m}\right)$ de $m$ entiers strictement positifs vérifiant l'égalité $p_{1}+\ldots+p_{m}=r+1$. On notera $s$ le schéma des drapeaux de type $\left(p_{1}, \ldots, p_{m}\right)$ de $E$ et $g: s \rightarrow x$ le morphisme structural. Le ${ }{ }_{S}$-Module $g$ " $E$ est alors muni $d$ une filtration canonique finie $0=F_{m} \longrightarrow F_{m-1} \longrightarrow \ldots \longleftrightarrow F_{0}=g^{*} E$ pour laquelle le quotient $E_{i}=F_{i-1} / F_{i}(1 \leqslant i<m)$ est localement libre 
de rang $p_{i}$.

On définit un morphisme d'anneaux

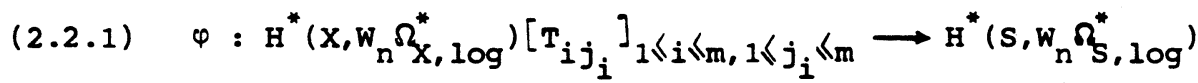
en convenant d'envoyer $H^{*}\left(x, w_{n} \Omega_{X}^{*}, \log \right)$ dans $H^{*}\left(S, w_{n} \Omega_{S, l o g}^{*}\right)$ par l'application $g^{*}$ et $T_{i j_{i}}$ sur la j-ième classe de Chern $c_{j_{i}}\left(E_{i}\right)$ de $E_{i}$.

Pour tout entier $i$, on déduit (par récurrence) de l'additivité $(1.1 .4)$ que

$$
c_{i}\left(g^{*} E\right)=\sum_{i_{1}+\ldots+i_{m}=i} c_{i_{1}}\left(E_{1}\right) \ldots c_{i_{m}}\left(E_{m}\right) .
$$

Ceci, joint à la fonctorialité (1.1.2) montre que $\varphi$ s'annule sur l'idéal $J_{X}$ de $H^{*}\left(X, W_{n} \Omega_{X, l o g}^{*}\right)\left[T_{i j_{i}}\right]_{1 \leqslant i \leqslant m, I \leqslant j_{i} \leqslant m}$ engendré par les éléments

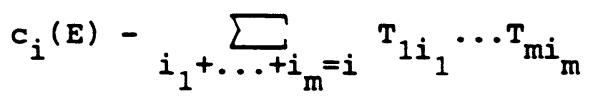

pour $i$ variable. D'où un morphisme d'anneaux

$$
\tilde{\varphi}: H^{*}\left(X, w_{n} \Omega_{X, \log }^{*}\right)\left[T_{i j_{i}}\right]_{\left(i, j_{i}\right.} / J_{X} \rightarrow H^{*}\left(S, w_{n} \Omega_{S, l o g}^{*}\right) .
$$

Théorème 2.2.5. Le morphisme $\tilde{\varphi}$ est un isomorphisme. En particulier $H^{*}\left(S, w_{n} \Omega_{S, l o g}^{*}\right)$ est un $H^{*}\left(x, w_{n} \Omega_{X}^{*}, \log \right)$-Module libre de rang $r ! /\left(p_{1} ! \cdots p_{m} !\right)$.

Démonstration : Elle est entièrement formelle et identique à celle donnée dans SGA 5, exp. 7, Prop. 5.4.

Terminons ce chapitre par une question : supposant $X$ propre (et lisse) sur $k$, a-t-on $A^{i}(x) \cap H^{i}\left(x, w_{n} \Omega_{x}^{i}, \log \right)^{0}=\{0\}$ où $A^{i}(x)$ désigne le sous-module de $H^{i}\left(X, w_{n} \Upsilon^{i} x, l o g\right)$ engendré par les classes $c_{i}(E)$ pour $E$ un $\theta_{X}$-Module localement libre de rang fini et où $\mathrm{H}^{i}\left(\mathrm{x}, \mathrm{w}_{n} \Omega^{\dot{i}} \mathrm{x}, \log \right)^{0}$ désigne la partie unipotente de $\mathrm{H}^{i}\left(\mathrm{x}, \mathrm{w}_{n} \Omega^{\dot{i}} \mathrm{x}, \log \right)^{\prime}$ (cf. [23, IV 3.3]) ? 


\section{Appendice : \\ Classes de Chern à supports.}

Nous allons brièvement indiquer comment on peut appliquer la construction de classes de Chern à supports de Gillet (cf. [12]) dans le cas de la cohomologie de Hodge-Witt logarithmique.

\subsection{Rappels.}

2.3.1. Si $X$ est un espace topologique et $G$ un faisceau de groupes sur $X$, on note $B_{G}$ le topos classifiant de $G$. La catégorie des objets abéliens dans ${ }^{B}{ }_{G}$ est équivalente à la catégorie des $z[G]$-Modules. Si $\underline{E}$ est un tel objet abélien, la $\underline{G}$-cohomologie de $\underline{E}$ est

$$
\text { (2.3.2) } \quad H^{i}(X, \underline{G}, \underline{E})=R^{i} \quad(X, \underline{\underline{G}})=E_{Z x}[\underline{G}](\underline{Z}, \underline{F})
$$

où $Z$ est le faisceau constant avec action triviale de $\underline{G}$ associé à $\mathbf{z}$.

2.3.3. Si $X$ est un schéma régulier et $Y$ un sous-schéma fermé (non nécessairement lisse) de $x$, on a (cf. [12]) un isomorphisme $(2.3 .4) \quad K_{*}^{Y}(X) \simeq K !(Y)$

où $K_{*}^{Y}(X)$ désigne la K-théorie à support dans $Y$ (cf. [12]) et $K$ : $(Y)$ la $K$-théorie de la catégorie des faisceaux cohérents sur $Y$.

\subsection{Le théorème principal.}

Théorème 2.4.1. Pour toute représentation $\rho: G \longrightarrow G L(E)$ de $\underline{G}$ dans un $\theta_{x}$-Module localement libre $\underline{F}$. il existe une unique théorie de classes de Chern $c_{i}(\rho) \in H^{i}\left(X, \underline{G}, w_{n} \Omega_{X}^{i}, \log \right)$ vérifiant les axiomes suivants

(2.4.2) Fonctorialité : si $f: Y \longrightarrow X$ est un morphisme entre $k$-schémas lisses et $\rho$ une représentation come ci-dessus sur $X$ et si $g$ est un homomorphisme $\underline{L} \longrightarrow \mathrm{f}^{*} \underline{G}$ de faisceaux en groupes 
sur $Y$, on a

$$
c_{i}\left(f^{*}(\rho) \circ g\right)=g^{*}\left(f^{*} c_{i}(\rho)\right) \text {. }
$$

(2.4.3) Normalisation : pour toute représentation comme ci-dessus, on a $c_{0}(\rho)=1$.

(2.4.4) Additivité : si

$$
0 \longrightarrow\left(\rho^{\prime}, \underline{F}^{\prime}\right) \longrightarrow(\rho, \underline{E}) \longrightarrow\left(\rho^{\prime \prime}, \underline{E}^{\prime \prime}\right) \longrightarrow 0
$$

est une suite exacte de représentations du faisceau en groupes $\underline{G}$. on a $c_{i}(\rho)=\sum_{m+q=i} c_{m}\left(\rho^{\prime}\right) c_{q}\left(\rho^{\prime \prime}\right)$.

Démonstration : Vu ce que l'on connait déjà sur la structure de la cohomologie de Hodge-Witt logarithmique d'un fibré projectif, il est très facile de recopier les constructions de Gillet ([12]. Chap. 2) en prenant garde au fait qu'il travaille avec la topologie de Zariski. Il faut donc remplacer son $\Gamma(i)$ par $R_{*} W_{n}{ }^{i} x_{1}, \log$ où $\varepsilon: x_{\text {et }} \rightarrow x_{\text {Zar }}$ est le morphisme canonique de topos. Les variantes simpliciales ne posent pas de problèmes.

\subsection{Classes de Chern à supports.}

On va construire des classes $c_{i, j}^{Y}: K_{j}^{Y}(x) \longrightarrow H_{Y}^{i-j}\left(x, w_{n} \Omega_{X, l o g}^{i}\right)$. Il suffit là encore de paraphraser Gillet (loc. cit.). Soient $x(-,-)$ le foncteur de Dold-Puppe et $z_{\infty}$ le foncteur de complétion de Bousfield et Kan. On a un isomorphisme (cf. [12])

(2.5.1) $\quad \mathrm{H}_{Y}^{-j}\left(x, z_{\infty} K\left(i, R \varepsilon, w_{n} \Omega_{X, l o g}^{i}\right)\right) \stackrel{\sim}{\longrightarrow} H_{Y}^{i-j}\left(x, w_{n} \Omega_{X}^{i}, \log \right)^{\prime}$.

Pour définir les $c_{i, j}^{Y}$, on procède alors comme dans le diagrame p. 229 de $[12]$. On observe que les classes de 2.4 permettent de construire des morphismes de faisceaux simpliciaux

$$
c_{i}: \operatorname{B.GL}\left(\theta_{X}\right) \longrightarrow K\left(i, R \varepsilon, W_{n} \Omega_{x, l o g}\right) .
$$

Le morphisme $c_{i, j}^{Y}$ est alors le composé du morphisme canonique $K_{j}^{Y}(X) \longrightarrow H_{Y}^{-j}\left(X, z \times Z_{\infty}\left(B \cdot G L\left(\theta_{X}\right)\right)\right.$, de $H_{Y}^{-j}\left(Z_{\infty} c_{j}\right)$ et de l'isomorphisme $(2.5 .1)$.

Proposition 2.5.3. Soit $f: Y \rightarrow X$ un morphisme de k-schémas lisses avec $Z$ un sous-schéma fermé (non nécessairement lisse) de $X$, on a alors un diagramme commutatif 
M. GROS

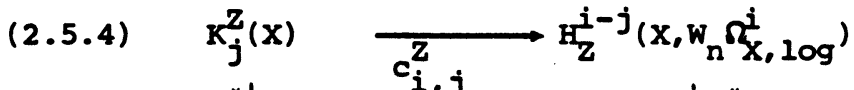

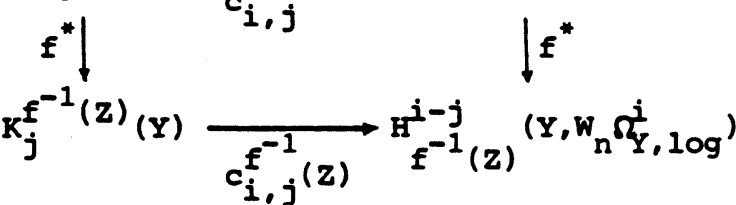

Démonstration : cf. Gillet ([12], Lemma 2.23$)$.

Remarque. Lorsque $\mathrm{Y}=\mathrm{X}$ et $\mathrm{j}=0$, on retrouve bien entendu les classes précédentes. 
CHAPITRE IV

APPLICATIONS

\section{Cohomologie d'un éclaté.}

\subsection{Cohomologie de Hodge-Witt d'un éclaté.}

Soient $X$ un $k$-schéma lisse et $i: Y \longrightarrow X$ une immersion fermée lisse définie par un idéal $J$. On notera $X^{\prime}=\operatorname{Proj}\left(\underset{n}{\oplus} J^{n}\right)$ l'éclaté de $X$ le long de $Y$. On a un diagramme cartésien fixant les autres notations

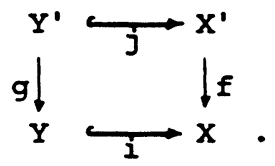

On a $Y^{\prime}=Y X_{X} X^{\prime}=P\left(J / J^{2}\right)$, et $J \cdot \theta_{X^{\prime}}=\theta_{X^{\prime}}(1)$ est $I^{\prime}$ idéal définissant $l^{\prime}$ immersion fermée $j \cdot j^{*} \theta_{X}(1)=\theta_{Y},(1)$ est le faisceau canonique sur le fibré projectif ' $Y^{\prime}$.

On va calculer $R_{*} W_{n} \Omega_{X^{\prime}}$.

Pour tout morphisme $h: z \longrightarrow T$ de $k$-schémas lisses, on pose (1.1.2) $\mathrm{R}^{+} \mathrm{h}, \mathrm{W} \Omega_{Z}^{*}=:\left.\operatorname{RHOm}\left(\theta \leftarrow \mathrm{R}_{\mathrm{T}}, \mathrm{W} \Omega_{\mathrm{Z}}^{*} \leftarrow \mathrm{W} \Omega_{\mathrm{T}}^{*}\right)[-1]\right|_{\mathrm{T}}$.

C'est un objet de la catégorie dérivée de la catégorie des $R_{T}$-Modules notée $D\left(R_{T}-\right.$ Mod) où $l$ 'on a considéré ( $W \Omega_{Z}$, W $W \Omega_{T}$. $\left.w \Omega_{T} \rightarrow h_{*} W \Omega_{Z}^{*}\right)=:\left(W \Omega_{Z}^{\circ} \leftarrow W \Omega_{T}^{*}\right)$ comme module sur le topos Top $(h)$ de la flèche $h$ ([19], 4 du Chap. III) annelé par $\left(R_{Z}-R_{T}\right):=$ $\left(R_{Z}, R_{T}, R_{T} \longrightarrow h, R_{Z}\right)$. On dispose donc d'un triangle distingué de $D\left(R_{T}-\right.$ Mod) (cf. Prop. 4.9 du Chap. III de [19])

$(1.1 .3)$

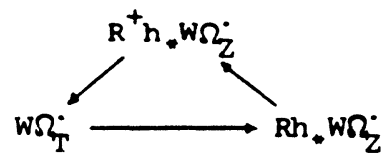

dépendant fonctoriellement de $\mathrm{h}$. 
Le diagramme (1.1.1) induit un morphisme dans $D\left(R_{X}-\right.$ Mod $)$ (1.1.4) $R^{+} f_{*} w \Omega_{X^{\prime}} \rightarrow i_{*} R^{+} g_{*} w \Omega_{Y^{\prime}}, \cdot$

Théorème $1.1 .5 .(1.1 .4)$ est un isomorphisme.

Avant de démontrer ce théorème, montrons comment il permet de calculer $\mathrm{Rf}_{*} \mathrm{~W}_{\mathrm{X}}^{\circ}$, ・

$D^{\prime}$ après II 2.7 .1

$(1.1 .5) \quad f_{*} f^{*}=I d$

puisque $f$ est birationnel. On en déduit donc un isomorphisme

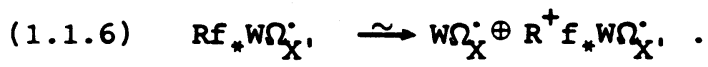

D'autre part, I 4.1.11 montre que l'on a un isomorphisme dans $D\left(R_{Y}-\operatorname{Mod}\right)$

(1.1.7) $\mathbf{R}^{+} g_{*} w \Omega_{Y^{\prime}}^{*}=\underset{j>0}{\oplus} w \Omega_{Y}^{*}(-j)[-j]$.

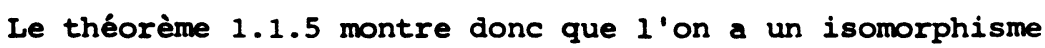

(1.1.8) $\mathrm{R}^{+} \mathrm{f}_{*} \mathrm{~W}_{\mathrm{X}^{\prime}} \stackrel{\sim}{\longrightarrow} \underset{j>0}{\oplus}{ }^{\mathrm{W}} \mathrm{W} \xi_{\mathrm{Y}}(-j)[-j]$.

Par suite

Théorème 1.1.9. On a un isomorphisme canonique dans $D\left(R_{X}-M o d\right)$

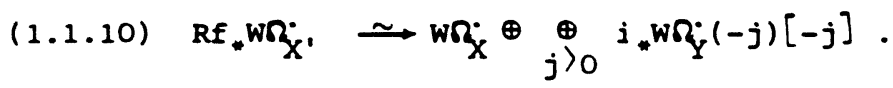

Corollaire 1.1.11. On a un isomorphisme canonique dans $D\left(w_{n} \Omega_{X}^{-M d g}\right)$

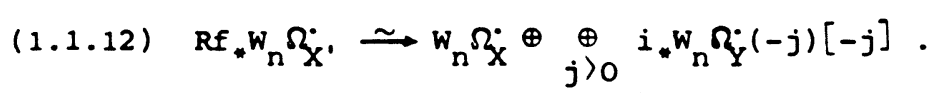

Démonstration : Il suffit d'appliquer le foncteur $R_{n} \stackrel{a}{\otimes}_{R}(-)$ à l'isomorphisme $(1.1 .13)$ et d'utiliser I'isomorphisme $I$ 4.1.15.

Venons-en à la démonstration du théorème 1.1.5. Soit $C$ le cône de (1.1.4), il s'agit de montrer que $c=0$. On va pour cela utiliser le même procédé que pour démontrer I 4.1.11. Il suffit donc de prouver, grâce à I 4.2.1, que

(1.1.14) $\quad R_{1} \otimes_{R} C=0$ 


\section{APPLICATIONS}

i.e. que $R_{1} \stackrel{n}{\otimes}_{R}(1.1 .4)$ est un isomorphisme.

Considérons alors pour tout morphisme $\mathrm{h}: \mathrm{Z} \longrightarrow \mathrm{T}$ de schémas sur une base arbitraire $s$ le module $\left(\Omega_{\mathrm{Z}}^{i}, \Omega_{\mathrm{T}}^{i}, \mathrm{~h}^{*} \Omega_{\mathrm{T}}^{i} \rightarrow \Omega_{\mathrm{Z}}^{i}\right)$ sur le topos annelé Top $(\mathrm{h})$ (annelé par $\left(\mathrm{O}_{\mathrm{Z}} \longleftarrow \mathrm{O}_{\mathrm{T}}\right):=\left(\mathrm{O}_{\mathrm{Z}}, \mathrm{O}_{\mathrm{T}}, \mathrm{O}_{\mathrm{T}} \longrightarrow \mathrm{h}_{*} \mathrm{O}_{\mathrm{Z}}\right)$, on pose

$$
\text { (1.1.15) } \mathrm{R}^{+} \mathrm{h}_{*} \Omega_{Z}^{i}=\mathrm{RHOM}_{\mathrm{H}}\left(0-\mathrm{O}_{\mathrm{T}^{\prime}} \Omega_{Z}^{i}-\Omega_{\mathrm{T}}^{i}\right)[-1] \mid \mathbf{T} \ldots
$$

C'est un objet de la catégorie dérivée de la catégorie des $\theta_{T}$-Modules notée $D\left(\theta_{T}-\right.$ Mod $)$. On dispose comme en $(1.1 .3)$ d'un triangle distingué dans $\mathrm{D}\left(\theta_{\mathrm{T}}-\mathrm{Mod}\right)$

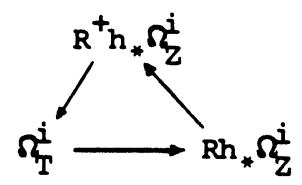

dépendant fonctoriellement de $\mathrm{h}$.

Ise morphisme $(1.1 .1)$ induit donc un morphisme

$$
\text { (1.1.17) } \mathrm{R}^{+} f_{*} \Omega_{X^{\prime}}^{i} \longrightarrow i_{*} \mathrm{R}^{+} g_{*} \Omega_{Y^{\prime}}^{i} \text {. }
$$

L'isomorphisme I (4.1.15) montre que I'on a des isomorphismes

(1.1.18) $\quad R_{1} \stackrel{\mathrm{Q}}{\otimes}_{\mathrm{R}} \mathrm{R}^{+} \mathrm{f}_{*} \mathrm{~W}_{\mathrm{X}^{\prime}} \stackrel{\sim}{\longrightarrow} \mathrm{R}^{+} \mathrm{f}_{*} \Omega_{\mathrm{X}^{\prime}}$,

(1.1.19) $\quad R_{1} \otimes_{R^{\circ}} R^{+} g_{*} w \Omega_{Y}^{*}, \stackrel{\sim}{\longrightarrow} R^{+} g_{*} \Omega_{Y}^{*}$.

On voit donc que $R_{1} \hat{\theta}_{R}(1.1 .4)$ sera un isomorphisme si $(1.1 .17)$ en est un pour tout $i \geqslant 0$.

\subsection{Cohomologie de Hodge d'un éclaté.}

On peut partir d'une situation un peu plus générale que celle décrite par le diagrame (1.1.1), à savoir la situation analogue où l'on considère les schémas en présence définis sur une base un schéma arbitraire $S$. On notera, pour tout S-schéma $T, \Omega_{T}$ le complexe de De Rham de $T / S$. On veut donc prouver que (1.1.17) est un isomorphisme. Vu la définition des deux membres, il suffit pour cela de prouver le théorème suivant : 
M. GROS

Théorème 1.2.1. Pour tout $i \in N$ et tout $q>1$, les flèches naturelles

$(1.2 .2)$

$$
\Omega_{x}^{i} \rightarrow f_{*} \Omega_{x}^{i}
$$

(1.2.3) $\quad R^{q_{f_{*}}} \Omega_{X^{\prime}}^{i} \longrightarrow i_{*} R^{q_{g_{*}}} \Omega_{Y^{\prime}}^{i}$

sont des isomorphismes.

Démonstration : On remarque tout d'abord que si $h: x_{1} \rightarrow x$ est un morphisme étale, le carré cartésien défini par l'éclatement de $x_{1}$ le long du sous-schéma fermé défini par $J_{1}=J \cdot O_{X_{1}}=h^{*} J$ se déduit de (1.1.1) par le changement de base $h$ et que $\Omega_{x_{1}}^{i}=h^{*} \Omega_{x}^{i}$ et de même pour $Y_{1}, X_{1}^{\prime}, Y_{1}^{\prime}$ puisque $h$ est étale.

Si l'on prouve le théorème 1.2 .1 pour un éclatement, il sera donc vrai pour tout autre éclatement défini par un changement de base étale $\mathrm{X}_{1} \longrightarrow \mathrm{X}$. La question étant locale au voisinage de $\mathrm{Y}$, on peut donc supposer que $X=s\left[t_{1}, \ldots, t_{s}, x_{1}, \ldots, x_{r+1}\right]$ et $Y$ défini par les équations $x_{1}=\ldots=x_{r+1}=0$. Posons $z=s\left[x_{1}, \ldots, x_{r+1}\right]$, on a alors $X=Y \times Z \quad\left(Y \propto A_{S}^{s}\right)$.

Le carré $(1.1 .1)$ est le produit par $Y$ du carré cartésien

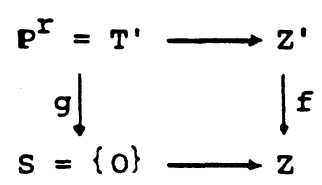

défini par l'éclatement de la section nulle de $\mathrm{Z}$. On a donc

$$
\text { (1.2.5) } \begin{aligned}
\Omega_{X}^{*} & =\Omega_{Y}^{*} \otimes \Omega_{Z}^{*} \\
\Omega_{X^{\prime}}^{*} & =\Omega_{Y}^{*} \otimes \Omega_{Z^{*}}^{*} \\
\Omega_{Y^{\prime}}^{*} & =\Omega_{Y}^{*} \otimes \Omega_{T^{\prime}}^{*} .
\end{aligned}
$$

d'où

$$
\begin{aligned}
& (1.2 .6) \quad \operatorname{Rf}_{*} \Omega_{X^{\prime}}^{*}=\Omega^{*} \otimes \mathrm{Rf}_{*} \Omega_{Z^{\prime}}^{*} \\
& \mathrm{Rg}_{*} \Omega_{Y^{\prime}}^{*}=\Omega_{Y^{*} \otimes \mathrm{Rg}_{*} \Omega_{T^{\prime}}^{*} .}
\end{aligned}
$$


Les morphismes $(1.2 .2)$ et $(1.2 .3)$ sont donc sommes, pour $j \geqslant 0$, des morphismes respectivement

$$
\text { (1.2.7) } \Omega_{Y}^{j} \otimes\left(\Omega_{Z}^{i-j} \longrightarrow f_{*} \Omega_{Z}^{i-j}\right)
$$

et

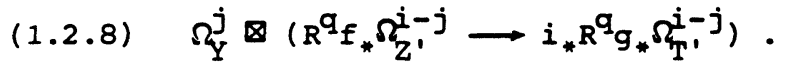

Il suffit donc de prouver le théorème 1.2.1 dans la situation (1.2.4) on peut donc supposer $Y=S$.

Considérons la filtration de Koszul de $\Omega_{X}^{i}$,

$$
\text { (1.2.9) } \quad F i l{ }^{j} \Omega_{X^{\prime}}^{i}=\operatorname{Im}\left(f^{*} \Omega_{X^{j}}^{j} \Omega_{X^{\prime}}^{i-j} \rightarrow \Omega_{X^{\prime}}^{i}\right) \text {. }
$$

Lemme 1.2.1.0 i) On a une suite d'inclusions

$$
\begin{aligned}
& 0=F_{i l}^{i+1} \Omega_{X^{\prime}}^{i} \subset F_{i l}{ }^{i} \Omega_{X^{\prime}}^{i} \subset \Omega_{X^{\prime}}^{i}(i-1) \subset F^{\prime} I^{i-1} \Omega_{X^{\prime}}^{i} \subset \Omega_{X^{\prime}}^{i}(i-2) \subset \ldots \subset \\
& F_{i l}{ }^{1} \Omega_{X^{\prime}}^{i} \subset \Omega_{X^{\prime}}^{i} . \\
& \quad \text { ii) Posons Fil }{ }^{j+(1 / 2)} \Omega_{X^{\prime}}^{i}=\Omega_{X^{\prime}}^{i}(j) . \text { On a des isomorphismes }
\end{aligned}
$$
canoniques
a) $g r^{j} \Omega_{X}^{i} \stackrel{\sim}{\sim} \Omega_{Y}^{i-1}(j)$
( $j$ entier, $1 \leqslant j \leqslant i-1)$,
b) $g r^{j+(1 / 2)} \Omega_{X^{\prime}}^{i} \simeq \Omega_{Y}^{i},(j)$
( $j$ entier, $1 \leqslant j \leqslant i-1$ )

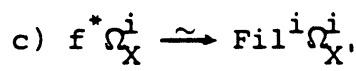
d) $\Omega_{X^{\prime}}^{i} / F i I^{1} \Omega_{X^{\prime}}^{i} \stackrel{\sim}{\longrightarrow} \Omega_{Y}^{i}$.

Démonstration : Rappelons tout d'abord que $X^{\prime}$ est le sous-schéma fermé de $\mathbf{P}_{X}^{r}$ défini par les équations $x_{i} y_{j}-x_{j} Y_{i}=0$, où $\left(y_{1}, \ldots, y_{Y+1}\right)$ sont des coordonnées homogènes sur $P_{X}^{r}$. et que la trace de $X^{\prime}$ sur $l^{\prime}$ ouvert affine de $P_{X}^{r}$ défini par $Y_{i} \neq 0$ est $l$ 'espace affine $s\left[z_{1}, \ldots, z_{i-1}, x_{i}, z_{i+1}, \ldots, z_{r+1}\right]$, la projection $f \mid x \cdot \cap U_{i}$ étant alors induite par l'homomorphisme d'anneaux $x_{i} \longrightarrow x_{i}, x_{j} \longrightarrow x_{i}{ }_{j}(j \neq i)$. Il.suffit de vérifier les inclusions de i) sur chaque $U_{i}$, et quitte à faire une permutation de coordonnées, on peut se borner au cas $i=1,\left(d x_{1}, d z_{2}, \ldots, d z_{r+1}\right)$ forment alors une base de $\Omega^{1}, \mid U_{1} \cap X^{\prime}$, et l'application canonique $\mathrm{f}^{*} \Omega_{\mathrm{X}}^{1} \longrightarrow \Omega_{\mathrm{X}}^{1}$, est donnée, en restriction à $v_{1}$, par $\mathrm{dx} \mathrm{x}_{1} \longmapsto \mathrm{dx}$ 
$d x_{i} \longmapsto x_{1} d z_{i}+z_{i} d x_{1}$ (i $>1$ ). C'est donc une application injective. Il en est donc de même de sa puissance extérieure $f^{*} \Omega_{X}^{i} \rightarrow \Omega_{X}^{i}$, d'où ii) c).

D'autre part, la restriction de $\theta_{X^{\prime}}(1)$ à $X^{\prime} \cap U_{1}$ est l'idéal principal $\left(x_{1}\right)$ et $Y^{\prime} \cap U_{1}$ est $I^{\prime}$ ouvert affine de $P_{S}^{r}$ de coordonnées affines $\left(z_{2}, \ldots, z_{r+1}\right)$.

Cette description rend évidentes les inclusions de i) et montre que la projection naturelle $\Omega_{X^{\prime}}^{i} \longrightarrow \Omega_{Y^{\prime}}^{i}$ induit l'isomorphisme ii) d).

Reste à prouver $i i)$ a) et ii) b). Considérons pour cela la suite exacte de $\theta_{Y}$-Modules localement libres de type fini

$(1.2 .11) \quad 0 \longrightarrow I / I^{2} \rightarrow \Omega_{X}^{1}, \otimes \theta_{Y}, \longrightarrow \Omega_{Y}^{1} \rightarrow 0$

où $I=\theta_{X},(1)$.

On en déduit une suite exacte

$(1.2 .12) \quad 0 \longrightarrow I / I^{2} \otimes \Omega_{Y^{\prime}}^{i-1} \longrightarrow \Omega_{X^{\prime}}^{i} \otimes \theta_{Y^{\prime}} \rightarrow \Omega_{Y^{\prime}}^{i} \rightarrow 0$.

soit, en tensorisant par $\theta_{X^{\prime}}(j-1)$

$(1.2 .13) \quad 0 \longrightarrow \Omega_{Y^{\prime}}^{i^{-1}}(j) \rightarrow \Omega_{X^{\prime}}^{i}(j-1) / \Omega_{X^{\prime}}^{i}(j) \longrightarrow \Omega_{Y^{\prime}}^{i}(j) \rightarrow 0$

(où $I^{\prime}$ on a identifié canoniquement $I / I^{2} \otimes \Omega_{Y^{\prime}}^{i-1}$ et $\Omega_{Y^{\prime}}^{i-1}(1)$ ). La flèche $\Phi$ est la flèche qui, sur l'ouvert $Y \cdot \cap U_{1}$, envoie $x_{1}^{j} d z_{j_{1}} \ldots d z_{j_{i}-1}$ sur $x_{1}^{j-1} d x_{1} d z_{j_{1}} \ldots d z_{j_{i}-1} \cdot L^{\prime i m a g e ~ d e ~} \Phi$ est donc $F i{ }^{j} R_{X}^{i}, / R_{X}^{i},(j)$, ce qui prouve a) et b) et conclut la preuve de 1.2.10.

Terminons la preuve de 1.2.1. D'après $1.2 .10 \mathrm{ii)}$ a) et ii) b) et (SGA 7, XI 1.1), on a

(1.2.14) $\operatorname{Rf}_{4 g^{j}} \Omega_{X^{\prime}}^{i}=0$ pour $1 \leqslant j \leqslant i-(1 / 2)$.

D'autre part, d'après (SGA 6, VII 3.5), on a un isomorphisme

$(1.2 .15) \theta_{X} \stackrel{\sim}{\sim} f_{*} \theta^{\prime}$

ce qui joint à ii) c) montre que

(1.2.16) Rf $F^{\prime} I^{i} R_{X^{\prime}}^{i}=\Omega_{X}^{i}$. 


\section{APPLICATIONS}

Les inclusions du lemme 1.2.10 i) et ii) d) prouvent donc que l'on a un triangle distingué

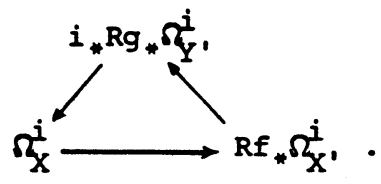

D'où le théorème 1.2 .1 en prenant la suite exacte longue de cohomologie correspondante.

\subsection{Cohomologie logarithmique d'un éclaté.}

Reprenons la situation décrite par le diagrame (1.1.1). Appliquant le foncteur $R^{\prime \prime} \underline{H o m}_{X^{\prime}} / p^{\cdot}\left(w R_{X}^{*} / p^{\cdot},-\right)$ à $I^{\prime}$ isomorphisme de $\mathrm{R}_{\mathrm{X}} / \mathrm{p}^{\cdot}$-pro-Modules

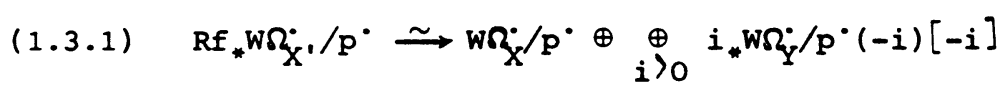

déduit de (1.1.10) par application du foncteur $(-) / \mathrm{p}^{*}$, on obtient le Théorème 1.3 .2 . On a un isomorphisme canonique dans $\mathrm{D}\left(\right.$ W. $\Omega_{\mathrm{X}, \mathrm{log}}^{*}$-pro-Mod )

$$
\text { (1.3.3) Rf,W. } \Omega_{X^{\prime}, \log }^{*} \stackrel{\sim}{\sim} \cdot \Omega_{X, \log }^{*} \underset{i>_{0}^{\oplus}}{\oplus} i_{*} W \cdot \Omega_{Y, \log }^{*}(-i)[-i] \text {. }
$$

Démonstration : Il suffit d'appliquer I (3.2.6), (3.2.8).

Corollaire 1.3.4. On a un isomorphisme canonique dans $D\left(W_{n} \Omega_{x, l o g}^{*}{ }^{-M o d}\right)$ $(1.3 .5)$

$$
R f w_{n} \Omega^{*} X^{\prime}, \log \stackrel{\sim}{\longrightarrow} w_{n} \Omega_{X, \log }^{*} \underset{i>0}{\oplus} i, w_{n} \Omega_{Y, \log }^{*}(i)[-i] \text {. }
$$

Démonstration : Il suffit de tensoriser l'isomorphisme (1.3.3) par $\mathrm{z} / \mathrm{p}^{\mathrm{n}} \otimes(-)$ et d'utiliser $\mathrm{l}$ 'isomorphisme $\mathrm{I}(1.3 .4)$.

Corollaire 1.3.6. On a, pour tout $j$, un isomorphisme de $\pi / p^{n}$-Modules (1.3.7) $H^{j}\left(X^{\prime}, w_{n} \Omega^{*} X^{\prime}, \log \right) \stackrel{\sim}{\sim} H^{j}\left(x, w_{n} \Omega_{X, l o g}^{*}\right) \oplus \underset{i>0}{\oplus} H^{j-i}\left(Y, w_{n} R^{*}-i\right.$ qui à $x \in H^{j}\left(x^{\prime}, w_{n} \Omega^{*},, l o g\right)$ associe $f_{*} x \oplus j^{*}\left(1-f^{*} f_{*}\right)(x), l^{\prime}$ isomorphisme réciproque étant donné par $\left(x, x^{\prime}\right) \longmapsto f^{\prime \prime} x+x^{\prime} \quad$ (on a identifié $\operatorname{Ker}\left(E_{*}\right)$ et $\underset{i>0}{\oplus} H^{j-i}\left(Y, W_{n} \Omega^{*}, \log ^{\prime}\right)$ grâce à $\left.j^{*}\right)$. 
Démonstration : Il suffit d'appliquer le foncteur $\mathrm{H}^{j}(\mathrm{x},-)$ à l'isomorphisme $(1.3 .5)$.

\section{Formule de self-intersection.}

2.1. Soit $i: Y \longrightarrow X$ une immersion fermée lisse de codimension $r+1$. On notera $J$ l'idéal de $\theta_{X}$ définissant $Y$ dans $X$ et $N$ le faisceau localement libre $\mathrm{J} / \mathrm{J}^{2}$.

Théorème 2.1.1. Pour tout $y \in \mathrm{H}^{*}\left(\mathrm{Y}, \mathrm{W}_{\mathrm{n}} \Omega_{\mathrm{Y}, \log }^{*}\right)$, on a (2.1.2) $\quad i^{*} i_{*}(y)=y c_{r+1}(\grave{N})$.

Démonstration : Elle va nécessiter plusieurs préliminaires dont le Lemme 2.1.3. Soit $F$ le $\theta_{Y}$,-Module localement libre défini par la suite exacte

$(2.1 .4) \quad 0 \longrightarrow \mathrm{F} \longrightarrow \mathrm{g}^{*} \mathrm{~N} \longrightarrow \theta_{\mathrm{Y}^{\prime}}(1) \longrightarrow 0$

on a alors

$$
(2.1 .5) \quad g_{*}\left(c_{Y}(\check{F})\right)=1 \text {. }
$$

Démonstration du lemme : Elle est formellement identique à celle de (SGA 5, VII, 8.4.1). L'additivité des classes de Chern, appliquée à la suite exacte (2.1.4), fournit l'égalité

$(2.1 .6)$

$$
g^{*}(c(N))=c(F)\left(1+c_{1}\left(\theta_{Y},(1)\right)\right.
$$

où $I^{\prime}$ on a noté $c(N)=\underset{i \geqslant 0}{\oplus} c_{i}(N) \in H^{*}\left(Y^{\prime}, W_{n} \Omega^{*},, \log \right)^{\prime}$.

On en déduit donc

$$
(2.1 .7) \quad c(F)=g^{*}(c(N))\left(1+c_{1}\left(\theta_{Y},(1)\right)^{-1}\right.
$$

d'où

$$
\text { (2.1.8) } \quad c_{Y}(F)=\sum_{0 \leqslant i \leqslant r}(-1)^{r-i} g^{*}\left(c_{i}(N)\right)\left(c_{1}\left(\theta_{Y},(1)\right)^{r-i} .\right.
$$

Comme g. diminue les degrés de $I$ et que, d'après II (2.6.2) on a $g_{*}\left(c_{1}\left(\theta_{Y},(1)\right)^{r}\right)=1$, on en déduit $(2.1 .5)$ en utilisant la formule de projection II $(2.2 .6)$. 


\section{APPLICATIONS}

On en déduit en particulier que (en utilisant la formule de projection)

$(2.1 .9)$

$$
g_{*}\left(g^{*}(y) c_{r}(\check{F})\right)=y \text {. }
$$

Lemme 2.1.10. Soit $\hat{\mathbf{N}}$ le complété projectif du fibré normal de $Y$ dans $X$. On notera $s: Y \longleftrightarrow N$ la section canonique de la projection canonique $g_{1}: \mathrm{N} \longrightarrow \mathrm{Y}$. Alors

$(2.1 .11)$

$$
s_{*}(1)=c_{r+1}\left(\check{F}_{1}\right)=\sum_{i=0}^{r+1} g_{1}^{*}\left(c_{i}(\stackrel{N}{N}) c_{1}\left(\theta_{\hat{N}}(1)\right)^{r+1-i}\right.
$$

où $F_{1}$ est défini par la suite exacte

$$
0 \longrightarrow \mathrm{F}_{1} \longrightarrow \mathrm{g}_{1}^{*}\left(\mathrm{~N} \oplus \theta_{\mathrm{Y}}\right) \longrightarrow \theta_{N}(1) \longrightarrow 0
$$

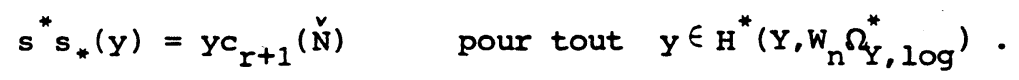

$(2.1 .13)$

$$
s^{*}(z)=g_{1 *}\left(z c_{r+1}\left(\check{F}_{1}\right)\right) \text { pour tout } z \in H^{*}\left(N, W_{n} \Omega_{N, l o g}^{*}\right) \text {. }
$$

Démnnstration : L'égalité du premier et du second membre de (2.1.11) n'est autre que le lemme 3 de [13]. L'égalité des deuxièmes et troisièmes membres est 1 'équivalent de (2.1.8).

Pour (2.1.12), on remarque que $l^{\prime}$ on peut supposer $y=1$ puisque

$$
s^{*} s_{*}(y)=s^{*} s_{*}\left(s^{*} g_{1}^{*}(y)\right)=s^{*}\left(s_{*}\left(s^{*} g_{1}^{*} y\right)\right)=y s^{*} s_{*}(1)
$$

grâce à la formule de projection $2.2 .6\left(\mathrm{~s}^{*} \mathrm{~g}_{1}^{*}=\mathrm{Id}\right)$.

Il s'agit alors de prouver que $s^{*}\left(c_{1}\left(\theta_{\hat{N}}(1)\right)=0\right.$. On remarque alors que si $\alpha: V(N) \longrightarrow \hat{N}$ désigne l'inclusion canonique, on a

$$
(2.1 .14 .1) \quad \alpha^{*}\left(c_{1}\left(\theta_{\hat{N}}(1)\right)=c_{1}\left(\theta_{V(N)}\right)=0\right.
$$

car $\alpha^{*} \theta_{\hat{N}}(1)=\theta_{\mathbf{v}(N)}$.

Prouvons (2.1.13). De la suite exacte définissant $F_{1}$ résulte que

$(2.1 .14 .2) \quad c_{1}\left(\theta_{\hat{N}}(1)\right) c_{r+1}\left(\check{F}_{1}\right)=c_{r+2}\left(g_{1} \ddot{N} \oplus \theta_{\hat{N}}\right)=0$.

on écrit alors $z=\sum_{i=0}^{r+1} g_{1}^{*}\left(a_{i}\right) c_{1}\left(\theta_{N}(1)\right)^{i}$. De $(2.1 .14 .1)$ résulte que $s^{*} z=a_{0} \cdot(2.1 .14 .2)$ montre que $z c_{r+1}\left(\check{F}_{1}\right)=g_{1}^{*}\left(a_{0}\right) c_{r+1}\left(\check{F}_{1}\right)$. D'où le résultat grâce à I'analogue de $(2.1 .9)$ pour $g_{1}$. 
Pour terminer la preuve de (2.1.2) nous allons avoir besoin des constructions suivantes. On note $\mathrm{u}: \mathrm{X} \rightarrow \mathrm{z}=\mathrm{X} \mathrm{x}_{\mathrm{k}} \mathrm{P}_{k}^{1}$ le morphisme $t \longmapsto(t, 0)$, on voit $Y$ comme un sous-schéma de $z$ grâce au morphisme $i_{1}=u i$. On éclate $Y$ dans $Z$, on obtient donc un diagramme cartésien

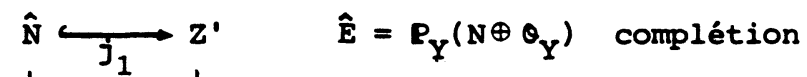

$$
\begin{aligned}
& g_{1} \underset{I_{1}}{\stackrel{I_{1}}{\longrightarrow}} \mathrm{Z}
\end{aligned}
$$

fixant les notations. On notera $\mathrm{S}: \mathrm{Y} \longrightarrow \mathrm{E}$ le même morphisme que dans 1 'énoncé 2.1 .10 .

L'inclusion $Y \longleftrightarrow X$ permet d'identifier $Y \times P_{k}^{1}=W$ à un sousschéma fermé de $z$. On note $W$ ' le sous-schéma de ' $Z^{\prime}$ éclaté de $W$ le long de $Y \propto Y X\{0\}$. Le morphisme canonique $f_{2}: W^{\prime} \rightarrow W$ est donc un isomorphisme. On fixe les autres notations relatives à $W^{\prime}$ par les diagrammes

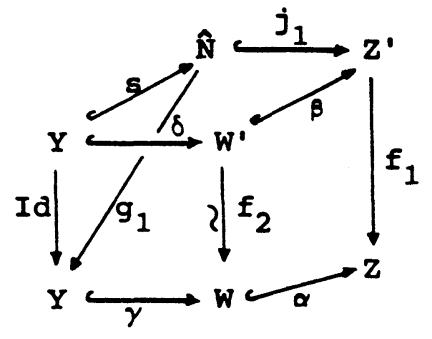

$(2.1 .17)$

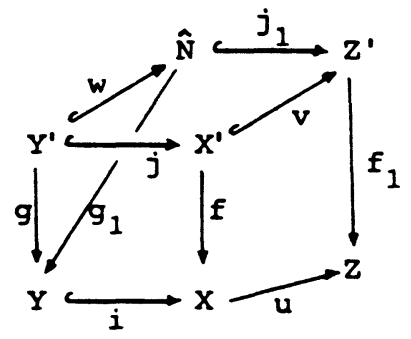

Lemme 2.1.18. Avec les notations précédentes, on a les relations

$$
\begin{aligned}
& (2.1 .19) \quad j_{1}^{*}\left(\beta_{*}(1)\right)=c_{r+1}\left(F_{1}^{v}\right) \\
& (2.1 .20) \quad j_{1}^{*}\left(v_{*}(1)\right)=c_{1}\left(\theta_{\hat{N}}(1)\right) \\
& (2.1 .21) \quad f_{1}^{*} u_{*}(1)=j_{1}(1)+v_{*}(1) .
\end{aligned}
$$




\section{APPLICATIONS}

Démonstration : Comme $j_{1}$ et $\beta$ (resp. $j_{1}$ et $v$ ) sont transversaux, on a

$$
\begin{aligned}
& (2.1 .22) \quad j_{1}^{*} \beta(1)=s_{*}(1) \\
& (2.1 .23) \quad j_{1}^{*} v_{*}(1)=w_{*}(1) .
\end{aligned}
$$

Les égalités (2.1.19) et (2.1.20) proviennent alors de (2.1.11) et de la compatibilité entre classe de cycle et classe de Chern. Pour (2.1.21), on remarque simplement que l'idéal inversible définissant $f_{1}^{-1}(x)$ dans $Z^{\prime}$ est le produit tensoriel des idéaux inversibles définissant $X^{\prime}$ et $\hat{N}$ dans $Z^{\prime}$ respectivement et que $\hat{N}$ et $X^{\prime}$ sont Tor-indépendants dans $Z^{\prime}$. D'où

$(2.1 .24) \quad f_{1}^{*} c_{1}\left(\theta_{Z^{\prime}}(X)\right)=c_{1}\left(\theta_{Z^{\prime}}\left(X^{\prime}\right)\right)+c_{1}\left(\theta_{Z^{\prime}}(\hat{N})\right)$.

La compatibilité entre classe de cycle et classe de Chern donne la formule $(2.1 .21)$.

Lerme 2.1.25. Pour tout $y \in H^{*}\left(\hat{N}, w_{n} \Omega_{\mathbb{N}}^{*} \log \right)$, on a

$(2.1 .26) \quad c_{r+1}\left(\check{F}_{1}\right) \cdot j_{1}^{*} j_{1 *}(y)=0$.

Démonstration : L'élément $y$ admet une unique décomposition

$(2.1 .27) \quad y=\sum_{i=0}^{r+1} a_{i} c_{1}\left(\theta_{\hat{N}}(1)\right)^{i}=\sum_{i=0}^{r+1} a_{i}\left(j_{1}^{*} v_{*}(1)\right)^{i}$.

La formule de projection pour le morphisme $j_{1}$ et l'égalité évidente $c_{1}\left(\theta_{\hat{N}}(1)\right) c_{r+1}\left(\check{F}_{1}\right)=0$ implique que

(2.1.28) $\quad c_{r+1}\left(\check{F}_{1}\right) j_{1}^{*} j_{1} *(y)=c_{r+1}\left(\check{F}_{1}\right) j_{1}^{*} j_{1} *\left(a_{0}\right)$.

On est donc ramené au cas où $y$ est de la forme $g_{1}^{*}(a)$. On a une unique décomposition

$$
j_{1}^{*} j_{1}\left(g_{1}^{*}(a)\right)=\sum_{i=0}^{r+1} g_{1}^{*}\left(b_{i}\right) c_{1}\left(\theta_{\hat{N}}(1)\right)^{i} \text {. }
$$

Utilisant que $c_{1}\left(\theta_{\hat{N}}(1)\right) c_{r+1}\left(\check{F}_{1}\right)=0$. on voit que 1 'on est ramené à voir que $g_{1}^{*}\left(b_{0}\right) c_{r+1}\left(\check{F}_{1}\right)=0$. On va voir que $b_{0}=0$. (2.1.29) se réécrit

$$
(2.1 .30) \quad j_{1}^{*} j_{1}\left(g_{1}^{*}(a)\right)=g_{1}^{*}\left(b_{0}\right)+z \cdot j_{1}^{*} v_{*}(1) .
$$

On applique alors $j_{1}$. et $l$ 'on utilise la formule de projection puis $(2.1 .21)$, on obtient 
M. GROS

$$
\text { (2.1.31) } j_{1 *} g_{1}^{*}\left(b_{0}\right)=j_{1 *} g_{1}^{*}(a) \cdot f_{1}^{*} u_{*}(1)-v_{*}(1) \cdot\left[j_{1 *} g_{1}^{*}(a)+j_{1 *}(z)\right] \text {. }
$$

Soit alors $\theta: f_{1}^{-1}(x \times\{1\}) \longleftrightarrow Z^{\prime} \quad l$ inclusion canonique. Elle vérifie $f_{1}^{*} u_{*}(1)=\theta_{*}(1)$ puisque les idéaux inversibles définissant $X \times\{0\}$ et $X X\{1\}$ dans $z$ sont isomorphes. On en déduit que

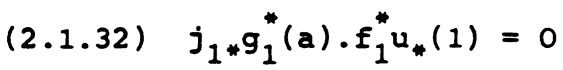

puisque $l^{\prime}$ on peut considérer $j_{1 *} g_{1}^{*}(a)$ (resp. $f_{1}^{*} u_{*}(1)$ ) comme un élément de $H_{N^{*}}^{*}\left(z^{\prime}, W_{n} \Omega_{Z^{\prime}, l o g}^{*}\right)$ (resp. $H_{f_{1}^{*}(x X\{1\})}\left(Z^{\prime}, W_{n} \Omega_{Z^{\prime}, l o g}^{*}\right)$ et que $\hat{N} \cap f_{1}^{-1}(x \times\{1\})=\varnothing$.

Par un argument de même nature, on voit que

$(2.1 .33) \quad B^{*} v_{*}(1)=0$

car $W^{\prime} \cap X^{\prime}=\varnothing \cdot D^{\prime}$ où

$(2.1 .34) \quad B^{*} j_{1} * g_{1}^{*}\left(b_{0}\right)=0$

grâce à (2.1.31)

$B$ et $j_{1}$ étant transverses, on en déduit

$(2 \cdot 1 \cdot 35) \quad 0=\delta_{*} s^{*} g_{1}^{*}\left(b_{0}\right)=\delta_{*}\left(b_{0}\right)$.

D'où, en appliquant $f_{2}$ *

$(2.1 .36) \quad \gamma_{*}\left(b_{0}\right)=0$.

Soit $b_{0}=0$ puisque $\gamma$. est injectif (puisque $\gamma$ admet une rétraction évidente).

Terminons la démonstration de (2.1.2). Soit $\mathrm{pr}: \mathrm{z} \longrightarrow \mathrm{x}$ la première projection, de sorte que $i=\operatorname{proi}_{1}$. On pose $\bar{x}=\operatorname{pr}^{*} i_{*}(y)$. on a

$(2.1 .37) \quad i^{*} i_{*}(y)=i_{1}^{*}(\bar{x})$

et $(2.1 .9)$ donne

$(2.1 .38) \quad i^{*} i_{*}(y)=g_{1}\left[g_{1}^{*} i_{1}^{*}(\bar{x}) \cdot c_{r+1}\left(\check{F}_{1}\right)\right]=g_{1} \cdot\left[j j_{1}^{*}(x) \cdot c_{r+1}\left(\check{F}_{1}\right)\right]$.

Soit alors $\mu=\beta \cdot f_{2}^{*}{ }^{*} y$. Le théorème de structure de la cohomologie d'un éclaté montre que $1^{\prime}$ on peut trouver $\omega^{\prime}$ et $\omega^{\prime \prime}$ tels que 
$(2.1 .39) \mu=f_{1}^{*} \mu^{\prime}+j_{1 * *^{*}}$

avec $f_{1 *^{\mu}}=\mu^{\prime}$. Or $f_{1 * \mu}=f_{1 *^{\beta}} f_{2}^{*} p^{*} y=\alpha_{*} f_{2 *} f_{2}^{*} p r^{*} y=\bar{x}$.

Reportant cette dernière égalité dans (2.1.39) puis dans (2.1.38), on voit que (utilisant $(2.1 .26)$ )

$(2.1 .40) \quad i^{*} i_{*}(y)=g_{1 *}\left[j_{1 *}(\mu) \cdot c_{r+1}\left(\breve{F}_{1}\right)\right]=s^{*} j_{1}^{*}(\mu)$

(la dernière égalité résultant de (2.1.13)). On remarque alors que

$(2.1 .41) \quad j_{1}^{*}(\mu)=j_{1}^{*} \beta{ }_{*}^{*} \mathrm{pr}^{*}(y)=s_{*} \delta^{*} f_{2}^{*} \mathrm{pr}^{*}(y)=s_{*}(y)$

puisque $\beta$ et $j_{1}$ sont transverses.

D'où (2.1.2) grâce à $(2.1 .12)$.

Corollaire 2.1 .42 . Sous les hypothèses du théorème 2.1 .1 , on a

$(2.1 .43) \quad i_{*}(x) i_{*}(y)=i_{*}\left(x y c_{I+1}(\check{N})\right)$.

Démonstration : La formule de projection II (2.2.6) entraine

$(2.1 .44) \quad i_{*}(x) i_{*}(y)=i_{*}\left(x i^{*} i_{*}(y)\right)$.

d'où $(2.1 .43)$ grâce à 2.1 .1 .

Proposition 2.1.44. ("formule-clef") Pour tout $y \in H^{*}\left(Y, W_{n} \Omega_{Y, l o g}^{*}\right.$ ). on a

$(2.1 .45) \quad f^{*} i_{*}(y)=j_{*}\left(g^{*}(y) c_{I}(\breve{F})\right)$.

Démonstration : Comme $f_{*} j_{*}=i_{*} g_{*}$, on en déduit de (2.1.9) que

$(2.1 .46) \quad i_{*}(y)=f_{*} j_{*}\left(g^{*}(y) c_{I}(\check{F})\right)$.

$D$ 'après 1.3 .6 , on $a$

$(2.1 .47) \quad j_{*}\left(g^{*}(y) c_{I}(\stackrel{F}{F})\right)=f^{*} x+x^{\prime}$ avec $f_{*} x^{\prime}=0$.

On va voir qu'en fait, $x^{\prime}=0$. Il suffit de montrer que $j_{*}\left(g^{*}(y) c_{r}(F)\right) \in \operatorname{Im} f^{*}$. Or, d'après 1.3 .6 , on a $\operatorname{Im} f^{*}=\operatorname{Ker}\left(j^{*}\left(1-f^{*} f_{*}\right)\right)$.

On a, compte tenu de (2.1)

$$
(2.1 .48) j^{*}\left(1-f^{*} f_{*}\right)\left(j_{*}\left(g^{*}(y) c_{r}(\breve{F})\right)=j^{*} j_{*}\left(g^{*}(y) c_{r}(\check{F})\right)-g^{*} i^{*} i_{*}(y)\right. \text {. }
$$

Utilisant que $j$ est de codimension 1 , on déduit de II (2.2.11) 
M. GROS

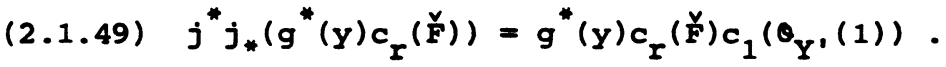

Ce qui joint à la formule de self-intersection permet d'écrire

$$
\begin{aligned}
j^{*} j_{*}\left(g^{*}(Y) c_{Y}(F)\right)-g^{*} i^{*} i_{*}(Y)= & g^{*}(Y)\left[c _ { I } ( F ) c _ { 1 } \left(\theta_{Y}(-1)-\right.\right. \\
& \left.g^{*}\left(c_{Y+1}(\tilde{N})\right)\right] .
\end{aligned}
$$

Cette dernière expression est nulle, car l'additivité des classes de Chern sur la suite exacte (2.1.4) fournit

$(2.1 .51)-g^{*}\left(c_{Y+1}(\stackrel{\Sigma}{N})\right)=c_{Y}(\stackrel{v}{F}) c_{1}\left(\theta_{Y},(1)\right)$.

On peut donc supposer $x^{\prime}=0$, on applique alors $f_{*}$ à (2.1.47). Comme on a $f_{*} f^{*}=I d$, on voit que $x=i(y)$, d'où (2.1.45).

\section{Classe de Chern et classe de cycle.}

3.1. On suppose que $x$ est un schéma quasi-projectif.

Proposition 3.1.1. Soit $Y$ un sous-schéma fermé (non nécessairement lisse) irréductible de $\mathrm{x}$, on $\mathrm{a}$

$$
c_{Y+1}\left(i_{*} \theta_{Y}\right)=(-1)^{r} r \operatorname{cl}(Y / X) \in H^{r+1}\left(X, w_{n} \Omega_{X, l o g}^{r+1}\right)
$$

$(\mathrm{r}+1=\operatorname{codim}(Y))$.

Démonstration : Les éléments en présence dans (3.1.2) sont images d'éléments appartenant (par définition pour la classe de cycle et grâce à l'appendice du chapitre III pour la classe de Chern) à $H_{Y}^{r+1}\left(x, w_{n} \Omega_{x, l o g}^{r+1}\right)$ par le morphisme canonique d'oubli du support. Ce groupe de cohomologie à support ne dépendant que de la partie lisse de $Y$, il suffit de démontrer (3.1.2) lorsque $Y$ est lisse. ce qui va résulter du

Théorème 3.1.3. ("Formule de Riemann-Roch sans dénominateurs") Pour tout $\theta_{Y}$-Module localement libre de rang $\varepsilon(G)$, on a

$$
c_{q+1}\left(i_{*} G\right)=i_{*}\left(p_{q+1}^{r+1}\left(\varepsilon(G), c_{1}(G), \ldots, c_{q-r}(G) ; c_{1}(N), \ldots, c_{q-r}(N)\right)\right.
$$

pour tout $q \in \mathbb{N}$ où $p_{q+1}^{r+1}\left(T_{0}, \ldots, T_{q-r} ; U_{1}, \ldots, U_{q-r}\right)$ est un certain polynôme universel à coefficients entiers dont la définition est rappelée dans $([24,1.1])$. 
La proposition 3.1 .1 résulte de 3.1 .3 appliqué appliqué au cas $G=\theta_{Y}, q=r$. En effet, on a $p_{q+1}^{r+1}=(-1)^{r} r l$.

Démonstration de 3.1 .3 : Elle est pratiquement identique à la formule de Riemann-Roch dans 1 'anneau de Chow donné dans [24]. Nous ne mentionnerons donc que les points qui diffèrent. On garde les notations de [24], qui sont compatibles avec les nôtres.

i) On n'a pas directement $f_{1}^{*} u_{*}(1)=j_{1 *}(1)+v_{*}(1)$ comme dans 1 'anneau de Chow, mais dans notre cas, il suffit de remarquer que l'idéal inversible définissant $f_{1}^{-1}(x)$ dans $Z^{\prime}$ est le produit tensoriel des idéaux inversibles définissant $X^{\prime}$ et $N$ dans $Z^{\prime}$ respectivement

(3.1.5) $\quad f_{1}^{*} c_{1}\left(\theta_{Z^{\prime}}(X)\right)=c_{1}\left(\theta_{Z^{\prime}}\left(X^{\prime}\right)\right)+c_{1}\left(\theta_{Z^{\prime}}(\mathrm{N})\right)$.

La compatibilité entre classe de Chern et classe de cycle donne l'égalité voulue.

ii) Pour voir que $c_{q+1}\left(j_{1 *}\left(g_{1}^{*}(G) . \omega\right) \cdot\left(f_{1}^{*} u_{*}(1)\right)=0\right.$, on note que 1 'inclusion canonique $\theta: \mathrm{f}_{1}^{-1}(\mathrm{x} x\{1\}) \longleftrightarrow \mathrm{z}$ ' vérifie $f_{1}^{*} u_{*}(1)=\theta_{*}(1)$ puisque les idéaux inversibles définissant $x \times\{0\}$ et $X X\{1\}$ dans $Z=X x_{k} P_{k}^{1}$ sont isomorphes et que $(X X\{1\}) \cap Y=\varnothing$. Comme on a trivialement

$$
\text { (3.1.6) } \quad \theta^{*}\left(c _ { q + 1 } \left(j _ { 1 * } \left(j_{1 *}\left(g_{1}^{*}(G) . \omega\right)=0\right.\right.\right. \text {. }
$$

le résultat en découle. 


\section{BIBLIOGRAPHIE}

[1] A. BEAUVILLE.- Traces et résidus en géométrie algébrique. Thèse de troisième cycle, orsay.

[2] P. BERTHELOT.- Cohomologie cristalline des schémas de caractéristique p) 0 (Lecture Notes in Math.. $n^{\circ} 407$, Springer-Verlag 1974).

[3] P. BERTHELOT.- Dualité plate, dans "Surfaces algébriques". Séminaire de Géométrie Algébrique d'Orsay, 1976-1978 (Lecture Notes in Math.. n' 868, Springer-Verlag 1981).

[4] P. BERTHELOT, L. ILLUSIE.- Classes de Chern en cohomologie cristalline (Notes aux C.R.A.S.P. du 22 et du 29 Juin 1970, Série A, t. 270).

[5] S. BLOCH, K. KATO.- p-adic etale cohomology. Preprint.

[6] S. BLOCH, A. OGUS.- Gersten's conjecture and the homology of schemes (Annales de I'E.N.S., 4e série, t. 7, 1974, p. 181 à 202).

[7] J.-L. COLLIOT-THELENE, J.-J. SANSUC, C. SOULE.- TOrsion dans le groupe de Chow de codimension deux (Duke Math. Journ. 50, 1983, 763-801).

[8] P. DELIGNE. - Théorème de Lefschetz et critères de dégénérescence des suites spectrales (Publications mathématiques de I'I.H.E.S. 35).

[9] T. EKEDAHL.- On the multiplicative properties of the De Rham-Witt complex. A paraitre dans Archiv flir Mathematik.

[10] T. EKEDAHL. - Travail en préparation.

[11] F. EI ZEIN.- Complexe dualisant et applications à la classe fondamentale d'un cycle (BSMF, Mémoire $n^{\circ} 58,1978$ ).

[12] H. GILLET.- Riemann-Roch theorems for higher algebraic $\mathrm{K}$-theory (Advances in Math. 40, 203-289 (1981)).

[13] A. GROTHENDIECK.- La théorie des classes de Chern (BSMF 86 , 1958, 137-154).

[14] A. GROTHENDIECK.- Classes de Chern et représentations linéaires des groupes discrets dans "Dix exposés sur la cohomologie des schémas (North-Holland, Masson).

[15] O. GABBER.- Lettre à Bloch du 11 Janvier 1982. 


\section{BIBLIOGRAPHIE}

[16] O. GABBER.- Gersten's Conjecture for some complexes of vanishing cycles. Preprint.

[17] . R. HARTSHORNE.- Residues and Duality (Lecture Notes in Math., $\mathrm{n}^{\circ} 20$, Springer-Verlag 1966).

[18] R. HARTSHORNE.- On the De Rham cohomology of algebraic varieties (Publications mathématiques de I'I.H.E.S. 45, 1976 5-99).

[19] L. ILLUSIE.- Complexe cotangent et déformations (Lecture Notes in Math., no 239 et 283, Springer-Verlag 1971 et 1972).

[20] L. ILLUSIE.- Complexe de De Rham-Witt (Astérisque 63, 1979, 83-112).

[21] L. ILLUSIE.- Complexe de De Rham-Witt et cohomologie cristalline (Annales de l'E.N.S., 4e série, t. 12, 1979, 501-661).

[22] L. ILLUSIE.- Finiteness, duality, and Kunneth theorems in the cohomology of the De Rham-Witt complex.

[23] L. ILLUSIE, M. RAYNAUD.- Les suites spectrales associées au complexe de De Rham-Witt (Publ. math. de l'IHES $n^{\circ} 57$ p. 73-212, 1983).

[24] J.-P. JOUANOLOU.- Riemann-Roch sans dénominateurs (Inventionnes Math. 11, 15-26 (1970)).

[25] K. KATO, S. SAITO.- Unramified class field theory of arithmetical surfaces (Annals of Math. 118 (1983), 241-275).

[26] N. KATZ.- Crystalline cohomology, Dieudonné Modules, and Jacobi Sums (Automorphic forms, Tata Institute, Bombay, 1979, 165246).

[27] B. MAZUR, L. ROBERTS.- Local Euler characteristics (Invent. Math. $9(1970), 201-234)$.

[28] J.S. MILNE.- Duality in the flat cohomology of a surface (Annales de I'E.N.S. 4e série, t. 9 (1976), 171-202).

[29] J.S. MILNE. - Zero cycles on algebraic varieties in non zero characteristic : Rotjman's theorem (Compositio Mathématica, vol. 47 (1982), 271-287).

[30] J.S. MILNE.- Values of zeta functions of varieties over finite fields. Preprint.

[31] N.1. NYGAARD. - Slopes of powers of Frobenius on crystalline cohomology (Annales de l'E.N.S. 4e série, t. 14 (1981), 369-401).

[32] A. OGUS.- Hodge cycles and crystalline cohomology dans "Hodge cycles. Motives and Shimura varieties" (Lecture Notes in Math. 900, Springer-Verlag 1982). 